\title{
Isotope - Based Material Science
}

\author{
Vladimir. G. Plekhanov \\ Computer Science College, Erika Street 7a, Tallinn, 10416,Estonia \\ *Corresponding Author: plekhanov@iati.ee
}

Copyright (C2013 Horizon Research Publishing All rights reserved.

\begin{abstract}
As is well - known isotopes of a given element have identical numbers of protons but differ in the number of neutrons making up their nuclei. Particularly, in crystalline solids, this difference in nuclear mass most directly affects vibrational phenomena, i.e. phonon frequencies and all phonon - related properties. These include exciton binding energy, electronic band gaps, lattice constant, local and crystalline vibrational modes, self - diffusion in bulk material as well as isotope superlattices (see, e.g. reviews [1,2]). The experience of the past shows that throughout constant technology improvement electronics(optoelectroelectronics)has become more reliable, faster, more powerful, and less expensive by reducing the dimensions of integrated circuits. These advantages are the driver for the development of modern microelectronics. The long - term goal of this development will lead to nanoelectronics. Advancing to the nanoscale is not just a step toward miniaturization, but requires the introduction and consideration of many additional phenomena. at the nanoscale, most phenomena and processes are dominated by quantum physics and they exhibit unique behavior. Nanotechnology includes the integration of man - made nanostructures into large material components of system (see, e.g. [3, 4]). Nanoscience and nanotechnology are concerned with materials, structures and systems whose components exhibit novel and significantly modified physical, chemical properties due to the nanoscale sizes. New direction of nanosciene is isotopetronics, who is studied the more low - dimensional size, as a rule the sizes of the sample of isotopetronis compare to the atomic size. Over the last five decades the large number of experimental and theoretical studies of isotopetronics have created the new branch of material science, which is called the isotope - based material science. Isotopetronics may find applications in quantum computing, nanoscience and spintronics. This review contains a brief introduction to the isotope - based material science.
\end{abstract}

Keywords Isotope, Semiconductor, Insulator, Excitons, Phonons

\section{Introduction}

Most of physical properties of solid depend on its isotopic composition in some way or another. Soddy realized that there are mixtures of elements which cannot be separated by chemical means. He then postulated that such elements, usually obtained at the beginning XX century by radioactive transmutation, have the same intraatomic charge but different atomic masses. Soddy called them isotopes because they occupy the same place in the periodic table of D.I. Mendeleev. At about the same time Thomson [7] found the presence of neon in gas discharge tubes, with atomic masses 20 and 22 and the average mass 20.2, which agreed with the accepted mass of this element. Isotopes are classified into stable and the unstable species. Presently about 300 stable and 1000 radioactive isotopes are known. Some elements are isotopically (foe example, Co), while other consist of plenty of isotopes (for example, Sn has 10 stable isotopes with masses from 112 to 124; and Xe has 23 isotopes, 9 of which are stable (see, for example $\left.\left.\left[7^{a}, 8,9\right]\right)\right)$. The unstable isotopes are radioactive and widely used as tracers in medical diagnostics, drug development and tomographic imaging. There exists a variety of interesting natural phenomena that are related to the varying abundance of isotopes. Many of them are not understood (see, also [10]). Each element found in our solar system has a naturally fixed isotopic composition. In this context, "natural" is related to the composition of elements found on planet Earth or in our solar system [11 - 13]. The natural abundance of all elements are tabulated, e.g. in $[9,10]$. Thus, isotopes represent a degree of freedom in the composition of matter while retaining an (almost) identical chemical behavior (see, also [14, 15]). Deviations of the isotopic abundance from natural one indicate an extrasolar origin of the material. Therefore, meteorites found on planet Earth can be distinguished from terrestrial material by comparison of the isotopic compositions [17]. Several methods for separating isotopes have been developed. They involve such diverse techniques as laser excitation, thermal diffusion, gas centrifugation 
and chemical exchange [18, 17]. As was indicated above, the main consumers of stable isotopes are pharmaceutical and biomedical industries. Chemicals containing stable isotopes can be kept on the shelf and activated by neutron irradiation shortly before using them in cancer therapy (for instance, ${ }^{88} \mathrm{Sn}+\mathrm{n}={ }^{89} \mathrm{Sn}$, which is radioactive and has found application in born cancer therapy). Isotopes have also found application in laser industry (He - Cd lasers with isotopically pure Cd produce a strong ultraviolet (UV) line, which is hard to obtain with natural Cd). Many more applications can be found by searching the Web for application of stable isotopes (see, also [16, 17]).

The availability of isotopically pure crystals with low carrier and impurity concentrations has allowed in the last three decades the investigation of isotope effects on lattice dynamical and electronic properties of solids [1, $2]$. The results of experimental and theoretical studies of the fundamental properties of the objects of research that earlier were simply in accessible (naturally with exception of $\mathrm{LiH}_{x} \mathrm{D}_{1-x}$ crystals) briefly are presented in the reviews $[1,2,19]$. The use of such objects allows the investigation of not only the isotope effects in lattice dynamics (elastic, thermal and vibrational properties) but also the influence of such effects on the electronic states via electron-phonon coupling (the renormalization of the band-to-band transition energy $\mathrm{E}_{g}$, the exciton binding energy $\mathrm{E}_{B}$ and the size of the longitudinal-transverse splitting $\Delta_{L T}$ ). The thermal conductivity enhancement in the isotopically enriched materials amounts (C; Ge; $\mathrm{Si}$ ) to almost $10 \%$ at room temperature and is close to a factor six at the thermal conductivity maximum around $20 \mathrm{~K}$ ( $\mathrm{Si}$ - case) (see also [2, 20]). The change in the lattice constant is $\Delta \mathrm{a} / \mathrm{a} \sim 10^{-3} \div 10^{-4}$, while the change $\delta \mathrm{c}_{i k}$ in the elastic constants amounts to several percent. In addition, crystals of different isotopic compositions possess different Debye temperatures. This difference between a LiH crystal and its deuteride exceeds hundred degrees. Of the same order of magnitude is the difference between Debye temperatures for diamond crystals. Very pronounced and general effects of isotopic substitution are observed in phonon spectra. The Raman lines in isotopically mixed crystals are not only shifted (the shift of LO phonon lines exceeds $100 \mathrm{~cm}^{-1}$ ) but are also broadened. This broadening is related to the isotopic disorder of a crystal lattice. It is shown that the degree of change in the scattering potential is different for different isotopic mixed crystals [21]. In the case of semiconducting crystals (C; Ge; $\mathrm{Si}$; $\alpha$-Sn etc), phonon scattering is weak, which allows one to successfully apply the coherent potential approximation (CPA) for describing shift and broadening of scattering lines in Raman spectra [1,2]. In the case of $\mathrm{LiH}$, the change in the scattering potential is so strong that it results in phonon localization, which is directly observed in experiments $[21,1]$.

Substituting a light isotope with a heavy one increases the interband transition energy $\mathrm{E}_{g}$ (excluding $\mathrm{Cu}$-salts) and the binding energy of the Wannier-Mott exciton $\mathrm{E}_{B}$ as well as the magnitude of the longitudinal-transverse splitting $\Delta_{L T}[20,22]$. The nonlinear variation of these quantities with the isotope concentration is due to the isotopic disordering of the crystal lattice and is consistent with the concentration dependence of line halfwidth in exciton reflection and luminescence spectra. A comparative study of the temperature and isotopic shift of the edge of fundamental absorption for a large number of different semiconducting and insulating crystals indicates that the main (but not the only) contribution to this shift comes from zero oscillations whose magnitude may be quite considerable and comparable with the energy of LO phonons. The theoretical description of the experimentally observed dependence of the binding energy of the Wannier-Mott exciton $\mathrm{E}_{B}$ on the nuclear mass requires the simultaneous consideration of the exchange of LO phonons between the electron and hole in the exciton, and the separate interactions of carriers with LO phonons (see also [1]). The experimental dependence $\mathrm{E}_{B} \sim \mathrm{f}(\mathrm{x})$ for $\mathrm{LiH}_{x} \mathrm{D}_{1-x}$ crystals fits in well enough with the calculation according to the model of large - radius exciton in a disordered medium; hence it follows that the fluctuation smearing of the band edges is caused by isotopic disordering of the crystal lattice. Due to zero-point motion, the atoms in a solid feel the anharmonicity [23] of the interatomic potential even at low temperatures. Therefore, the lattice parameters of two chemically identical crystals formed by different isotopes do not coincide heavier isotopes having smaller zero-point delocalization (as expected in a harmonic approximation) and smaller lattice parameters (an anharmonic effect). Moreover, phonon related properties such as thermal conductivity, thermal expansion or melting temperature, are expected to depend on the isotope mass (for details see [1]).

Our brief discussion, we start with a fact that phonon frequency are directly affected by changes of the average mass of the whole crystal or its sublattice (VCA - model), even if we look upon them as noninteracting particles, i.e., as harmonic oscillators. The direct influence of the isotope mass on the frequencies of coupled phonon modes may been used to determine their eigenvectors. Secondly, the mean square amplitude $\left\langle\mathrm{u}^{2}\right\rangle$ of phonons depend on the isotope masses only at low temperature, while they are determined by the temperature $\mathrm{T}$ only, once $\mathrm{T}$ becomes larger than Debye temperature. A refinement of these effects must take place when taking interactions among phonons into account. These interactions lead to finite phonon lifetimes and additional frequency renormalization. The underlying processes can be divided into two classes: 1) anharmonic interactions in which a zone center phonon decays into two phonons or more with wave-vector and energy conservation, and 2) elastic scattering in which a phonon scatters into phonons of similar energies but different wave - vectors. While the former processes arise from cubic and quartic terms in the expansion of lattice potential [23], the latter are due to the relaxed wave - vector conservation rule in samples that are isotopically disordered and thus not strictly translational invariant. Since the vast majority of compounds derive from elements having more than one stable isotope, it is clear that both processes are present most of the time. Unfortunately, their absolute sizes and relative importance cannot be predicted easily. However, isotope enrichment allows one to suppress the elastic scattering induced by isotope disorder. In contrast, the anharmonic phonon-phonon interaction cannot be suppressed, so that isotope-disorder-induced effects can only be studied against a background contribution from anharmonic processes. However, if one assumes that the two 
processes are independent of each other one can measure the disorder-induced renormalization by comparison of phonon energies and linewidth of isotopically pure samples with those gained from disordered ones.

The isotopic composition affects the band-gaps through the electron-phonon coupling and through the change of volume with isotopic mass. Although the electronic properties of different isotopes of a given atom are, to a very good approximation, the same, isotope substitution in a crystal modifies the phonon spectrum which, in turn, modifies the electron energy bands through electron-phonon interaction. Measuring the energy gaps in samples with different isotopic composition then yields the difference in the changes of the valence- and conduction band renormalization. The reason for the changes lies in the fundamental quantum-mechanical concept of zero - point motion - the vibrational energy that the atoms in the crystal have, even at low temperatures. If we excite an electron from one electronic state to another, we actually excite the whole crystal. In other words, we move the crystal from a ground state made up of low-energy electrons plus zero-point vibrations to an excited state in which there is one excited electron plus the zero-point vibrations of the crystal. The values of the zero-point energy in the two electronic states are slightly different because the vibrational frequencies depend on the chemical bonding, which is changed by exciting an electron. If the average mass of the vibrating atoms is increased, then the vibrational frequencies will be reduced. As a result, the difference in zero-point motions will be smaller, and the transition energy will therefore increase with increasing mass [1].

Solid materials are often doped, either intentionally or not, and the doping impurities are in many instances dominant factors in the optical spectroscopy of the material. Impurities can introduce electronic levels in the gap (and transitions between these levels) and can localize excitons forming bound excitons. They can also change the phonon spectrum in the material by introducing local vibrational modes, which result in new absorption and Raman transitions [24]. Vibrations of the isotopic mass of the impurity species will have a strong and direct effect in the frequency of the impurity local vibrations modes and may also have a small effecton the electronic binding energies of impurity levels (see, however below). The present review deals primarily with isotopic effect related to the host material and not the impurities. Thus, different characteristics depending on the isotope effect is formed the essence of the isotope - based material science.

\section{Electronic band structure}

The importance of the electronic theory of solids as embodied in band theory is that it provides us with clear means of understanding how solids may be insulators, semiconductors or metals. This dependence upon whether or not is a Fermi surface. The existence of a Fermi surface produces metallic behavior, whereas at 0K, if the filled electron levels are separated from vacant ones, we have insulating properties. If the separation is large, say $\geq 5$ $\mathrm{eV}$, the substance remains an insulator at temperature above $0 \mathrm{~K}$, whereas semiconducting properties arise if the filled and empty levels lie within $0-2 \mathrm{eV}$ of one another [25].

\subsection{Insulators}

$\mathrm{LiH}$ crystal is of $\mathrm{NaCl}$ structure type. The simple electronic structure of $\mathrm{Li}^{+}$and $\mathrm{H}^{-}$ions, having $1 \mathrm{~s}^{2}$ configuration, gives $\mathrm{LiH}$ a special place among the binary crystals and in many aspects allows it to serve as an ideal model for ionic compounds. The spectrum of one-electron states of crystal is determined by the solution of Schrodinger equation for the 'extra' particle (hole or electron) moving in the averaged field created by all the remaining electrons and nuclei (see, also [22]):

$$
\left[-\frac{\hbar^{2}}{2 m} \Delta+\mathrm{V}(\vec{r})\right] \Psi_{\vec{k}}(\vec{r})=\mathrm{E}_{n}(\vec{k}) \Psi_{\vec{k}}(\vec{r})
$$

where the notation is conventional, and $\mathrm{V}(\vec{r})$ is the periodic potential. The existing calculation techniques differ in the method of constructing the electron potential $\mathrm{V}(\vec{r})$, the approximation of the wave function $\Psi_{\vec{k}}(\vec{r})$, the ways of ensuring self-consistency, the reliance on empirical parameters, etc. In particular, two factors are especially important in case of ionic crystals: (1) the inclusion of exchange interaction, and (2) the inclusion of polarization of the electron and ion subsystems of crystal by the extra particle [25].

The one-electron potential of any many-electron system is nonlocal because of the exchange interaction between the electrons. It is very difficult to take this interaction into account. Because of this, the exact potential in the band theory is often replaced by the local potential of the form $\mathrm{V}_{\text {exchange }}(\vec{r}) \propto \alpha[\rho(\vec{r})]^{1 / 3}$, where $\rho(\vec{r})$ is the charge density function, and the constant $\alpha$ is selected in the range from 1 (Slater potential [26]) to 2/3 (Cohn-Sham potential) [22].

Two effects are associated with the local exchange. First, the results of calculation depend strongly on the numerical value of $\alpha$, and second, this approximation always underestimates the values of $\mathrm{E}_{g}$ and $\mathrm{E}_{v}$ (the width of the valence band). It is the low values of $\mathrm{E}_{v}$ obtained by many authors in the approximation of local exchange that are responsible for the wrong conclusion concerning the inapplicability of band theory to ionic dielectrics. These problems reflect the fundamental drawback of the one-electron approximation which does not take into account the reciprocal effect of the selected electron (hole) on the rest of the system. This effect consists in the polarization of the crystal by the particle, and is generally made up of two parts: the electron polarization (inertialess), and the lattice polarization (inertial). In the common optical phenomena, related to absorption or scattering of photons, the lattice polarization is not important, because the frequency of optical transitions is much higher than the average frequencies of phonons. The electron polarization is different. The extra particle (electron, hole) is regarded by 
this theory as the slowest particle in the system - in other words, all the remaining electrons adiabatically follow it. Hence it follows that the inertialess polarization definitely must be included in the calculation of energy spectrum. An important feature of ionic crystals is that the polarization energy $\mathrm{E}_{p}$ is of the same order of magnitude as the bandwidth. Such a correction obviously cannot be regarded as small. In the extreme case of particle at rest, the polarization energy can be calculated by methods of classical electrostatics (the Mott - Littleton method [25]), or by the newer and more accurate technique proposed by Fowler [27]. The value of $\mathrm{E}_{p}$ for AHC found by this method is 2-3 eV for each of the quasi-particles ( $\mathrm{E}_{p}>0$ for electrons, and $\mathrm{E}_{p}<0$ for holes). This implies that the inclusion of electron polarization will reduce the magnitude of $\mathrm{E}_{g}$ by $4-5 \mathrm{eV}$ [22]. By assumption, the electron bands are displaced rigidly, without changing the dispersion law $\mathrm{E}(\vec{k})$.

The simple electron structure of lithium hydride (combined with the negligibly small spin-orbital interaction) is very helpful for calculating the band structure: all electron shells can easily be taken into account in the construction of the electron potential. The first calculations of band structure of lithium hydride were carried out as early as 1936 by Ewing and Seitz [28] using the Wigner-Seitz cell method. This method consists essentially in the following. The straight lattice is divided into polyhedra in such a way that the latter fill the entire space; inside each polyhedron is an atom forming the basis of the lattice (Wigner-Seitz cells). The potential inside each cell is assumed to be spherically symmetrical and coinciding with the potential of free ion. This approximation works well for ions with closed shells. The radial Schrodinger equation in the coordinate function $\mathrm{R}_{l}(\vec{r})$ is solved within each selected cell, the energy being regarded as a parameter. Then the Bloch function is constructed in the form of expansion

$$
\Psi_{\vec{k}}(\vec{r})=\sum_{l=0}^{\infty} \sum_{m=-l}^{l} C_{l m}(\vec{k}) \mathrm{Y}_{l m}(\theta, \varphi) \mathrm{R}_{l}(\vec{r}, \mathrm{E})
$$

where $\vec{r}, \theta, \varphi$ are the spherical coordinates (with respect to the center of the cell); $\mathrm{Y}_{l m}$ are spherical functions. The coefficients $\mathrm{C}_{l m}(\vec{k})$ and the energy $\mathrm{E}(\vec{k})$ are found from conditions of periodicity and continuity on the boundaries of the cell. If $\vec{r}_{1}$ and $\vec{r}_{2}$ are the coordinates of two points on the surface of Wigner-Seitz cell, linked by the translation vector $\vec{R}_{l}$, then the boundary conditions are [14]

$$
\begin{aligned}
& \Psi_{\vec{k}}\left(\vec{r}_{2}\right)=\exp \left(i \vec{k} \vec{R}_{l}\right) \Psi_{\vec{k}}\left(\vec{r}_{1}\right) \\
& \nabla_{n} \Psi_{\vec{k}}\left(\vec{r}_{2}\right)=\exp \left(-i \vec{k} \vec{R}_{l}\right) \nabla_{n} \Psi_{\vec{k}}\left(\vec{r}_{1}\right)
\end{aligned}
$$

where $\nabla_{n}$ is the gradient normal to the surface of the cell. We see that the method of cells only differs from the problem of free atom in the boundary conditions. Owing to the complex shape of the cell, however, the construction of boundary conditions is a very complicated task, and this method is rarely used nowadays.

The method of plane associated waves (PAW) was used for calculating the band structure and the equation of state for $\mathrm{LiH}$ was used in Perrot [29]. According to this method, the crystal potential is assumed to be spherically symmetrical within a sphere of radius $\vec{r}_{s}$ described around each atom, and constant between the spheres (the so-called cellular muffin-tin (MT) potential). Inside each sphere, like in the Wigner-Seitz method, the solutions of Schrodinger equation have the form of spherical harmonics; outside the spheres they become plane waves. Accordingly, the basis functions have the form

$$
\Psi_{\vec{k}}(\vec{r})=\exp (i \vec{k} \vec{r}) \theta\left(\vec{r}-\vec{r}_{s}\right)+\sum a_{l m} \mathrm{Y}_{l m}(\theta, \varphi) R_{l}(E, \vec{r}) \theta\left(\vec{r}_{s}-\vec{r}\right),(5)
$$

where $\theta(\mathrm{x})=1$ at $\mathrm{x} \geq 0$, and $\theta(\mathrm{x})=0$ at $\mathrm{x}<0$. The coefficients $\mathrm{a}_{l m}$ can be easily found from condition of sewing on the boundary of the sphere. This is an important advantage of the PAW method over the method of cells. The calculations of Perrot [29] are self-consistent, and the local potential is used in the Cohn-Sham form. The correlation corrections were neglected. The method of Corringi-Cohn-Rostocker (CCR method), or the method of Green's functions, was used for calculating the band structure of LiH in Zavt et al. [30] (only concerned with the valence band) and in Kulikov [31]. Calculation of band structure of LiH in Grosso and Paravicini [32] was based on the wave function used in the method of orthogonalized plane waves (OPW) of the form

$$
\Psi_{\vec{k}}(\vec{r})=\exp (i \vec{k} \vec{r})-\sum_{c}\left\langle\exp (i \vec{k} \vec{r}) \mid \mathrm{X}_{c}\right\rangle X_{c}(\vec{r})
$$

where $\mathrm{X}_{c}$ are the atomic functions of state of the skeleton; $\left\langle\exp (i \vec{k} \vec{r}) \mid \mathrm{X}_{c}\right\rangle$ is the integral of overlapping of plane wave with skeleton function (see also [33]). The method of linear combination of local basis functions was applied to the calculation of band structure of $\mathrm{LiH}$ in Kunz and Mickish [34]. This method is based on constructing the local orbitals for the occupied atom states, based on certain invariant properties of the Fock operator. The main feature of local orbitals is that they are much less extensive than the atom orbitals. Importantly, the correlation correction is taken into account in Kunz and Mickish [34]. Owing to the high polarizability of hydrogen molecules, the correlation effect in lithium hydride is exceptionally strong. Yet another calculation of band structure of LiH was carried out in Zavt et al. [30] using the so-called method of extended elementary cell. This approach is based on the semiempirical techniques of the theory of molecules, and is much similar to the cluster calculations. Let us add that the cluster is selected in such a way that the quasi-molecular wave function transforms in accordance with the group symmetry of certain wave vectors in the Brillouin zone. This methods only yields the energy values at the points of high symmetry. We ought to mention also Hama and Kawakami [35], where, in connection with the study of high pressure effects on the transition $\mathrm{NaCl}$ - CsCI in lithium hydride, the band structure and the equation of state of the latter are analyzed in detail. The calculated band structures of LiH are compared in Fig. 1. We see that the overall picture given by various methods is generally the same, despite the vast spread of the transition energy values (see Table 1). Looking at the structure of the valence band we see that it is very similar 
to the s-band in the method of strong bond (see also [22]). This is surprising, given the strong overlap of the anion s-functions in lithium hydride. The wave functions in this band are almost entirely composed of the Is states of hydrogen ion. Different authors place the ceiling of the band either at point X or at point W of the Brillouin zone. Although in all cases the energies of the states $\mathrm{X}_{1}$ and $\mathrm{W}_{1}$ differ little $(\leq 0.3 \mathrm{eV})$, the question of the actual location of the top of the valence band may be important for the dynamics of the hole. Different calculations also disagree on the width of the valence band. For example, the width of the valence band in LiH without correlation is, according to Kunz and Mickish [34], $\mathrm{E}_{\mathrm{v}}=14.5 \mathrm{eV}$, and the value of $\mathrm{E}_{\mathrm{v}}$ is reduced to one half of this when correlation is taken into account. This shows how much the polarization of crystal by the hole affects the width of the valence band $\mathrm{E}_{\mathrm{v}}$. According to Perrot [29], the width of the valence band in $\mathrm{LiH}$ is $5.6 \mathrm{eV}$. The density of electron states in the valence band of $\mathrm{LiH}$ was measured in Betenekova et al. [36] and Ichikava et al. [37]. In Betenekova et al. the measurements were carried out with a magnetic spectrometer having the resolution of 1.5 $\mathrm{eV}$, whereas the resolution of hemispherical analyzer used in Ichikawa et al. [37] was 1.1 eV. From experimental data, the width of the valence band is $6 \mathrm{eV}$ according to Betenekova et al., and $6.3 \mathrm{eV}$ according to Ichikawa et al. Observe the good agreement with the calculated value of $\mathrm{E}_{\mathrm{v}}$ in this theory. Let us add also that the measured distribution of the electron density of states in the valence band of LiH exhibits asymmetry typical of s-bands (for more details see Betenekova et al. [36] and Ichikawa et al. [37]). The lower part of the conduction band is formed wholly by $\mathrm{p}$-states and displays an absolute minimum at point $\mathrm{X}$ which corresponds to the singlet symmetry state $\mathrm{X}_{4}$. The inversion of order of $\mathrm{s}$ and p-states in the spirit of LCAO method may be understood as the result of the s-nature of valence band. Mixing of s-states of the two bands leads to their hybridization and spreading, which changes the sequence of levels (see also [10] and references there). If we compare the structure of the conduction band with the p-band of the method of strong bond (see, also [39]), we see that the general structure and the sequence of levels are the same except for some minor details (the location of $\mathrm{L}_{3}$ level, and the behavior of $\mathrm{E}(\vec{k})$ in the neighborhood of $\Gamma_{15}$ ). In other words, the lower part of the conduction band in lithium hydride is very close to the valence $\mathrm{p}$-band of $\mathrm{AHC}$. The direct optical gap in $\mathrm{LiH}$ according to all calculations is located at $\mathrm{X}$ point and corresponds to the allowed transition $\mathrm{X}_{1}-\mathrm{X}_{4}$. The indirect transition $\mathrm{W}_{1}-\mathrm{X}_{4}$ ought to have a similar energy. According to the above calculations, the energies of these transitions differ by $0.03-0.3 \mathrm{eV}$. The different values of $\mathrm{E}_{g}$ for $\mathrm{LiH}$ obtained by different authors are apparently due to the various methods used for taking into account the exchange and correlation corrections (see above).

Table 1. Calculated energy values of some direct optical transitions in $\mathrm{LiH}$ reduced to the experimental value of $\mathrm{E}_{g}=5.0 \mathrm{eV}$.

$\begin{array}{lllll}\text { Transition } & 1 & 2 & 3,4 & 5 \\ \mathrm{~K}_{1}-\mathrm{K}_{3} & 6.9 & 7.5 & 6.5 & 6.4 \\ \mathrm{~W}_{1}-\mathrm{W}_{3} & 8.0 & 7.9 & 7.3 & 7.4 \\ \mathrm{~L}_{1}-\mathrm{L}_{2} & 9.2 & 9.6 & 9.0 & 9.1 \\ \mathrm{~W}_{1}-\mathrm{W}_{2}^{\prime} & 12.6 & 14.9 & 12.2 & \\ \mathrm{X}_{1}-\mathrm{X}_{5}^{\prime} & 12.9 & 13.8 & 13.6 & \\ \mathrm{~K}_{1}-\mathrm{K}_{4} & 14.7 & 16.1 & 15.0 & \\ \mathrm{~L}_{1}-\mathrm{L}_{3} & 19.7 & 20.9 & 20.7 & \\ \Gamma_{1}-\Gamma_{15} & 24.5 & 25.3 & 33.3 & \end{array}$

As follows from Table 1, the transitions at critical points in the low-energy region form two groups at 7 - 9 and 13-15 eV. Measurements of reflection spectra in the $4-25 \mathrm{eV}$ range at $5 \mathrm{~K}$ (Kink et al. [38]) and $4-40 \mathrm{eV}$ at 300 $\mathrm{K}$ throw new light on the results of calculations (see also review by Plekhanov [39]). The singularities occurring at 7.9 and $12.7 \mathrm{eV}$ in reflection spectra are associated in the above papers with the interband transitions $\mathrm{W}_{1}-\mathrm{W}_{4}$ and $\mathrm{X}_{1}-\mathrm{X}_{5}$ respectively.


Figure 1. Band structure of LiH crystal as calculated: [34] - 1; [29] - 2; [33] - 3.

From the standpoint of dynamics of quasi-particles, an important consequence of such band structure is the high anisotropy of the tensor of effective mass of electrons and (especially) holes. The estimated mass of electron in the neighborhood of $\mathrm{X}_{4}$ is, according to Kunz and Mickish [34], $\left(m_{e}\right)_{x} \simeq 0.3 \mathrm{~m}_{0}$ in the direction $\mathrm{X}-\Gamma$, and $\left(\mathrm{m}_{e}\right)_{y}=$ 
$\left(\mathrm{m}_{e}\right)_{z} \simeq 0.8 \mathrm{~m}_{0}$ in the direction $\mathrm{X}-\mathrm{W}$. Similarly, the mass of hole in the neighborhood of $\mathrm{X}_{1}$ is $\mathrm{X}-\Gamma$ in the direction $\left(\mathrm{m}_{e}\right)_{x} \simeq 0.55 \mathrm{~m}_{0}$ and about the same in the neighborhood of $\mathrm{W}_{1}$. It is assumed that the transverse components of $\mathrm{m}_{h}$ are greater by several orders of magnitude (Zavt et al. [30]). Note also that, according to Baroni et al. [33], the estimated masses of carriers are: $\mathrm{m}_{e l}=0,121 ; \mathrm{m}_{e t}=0,938 ; \mathrm{m}_{h l}=0,150 ; \mathrm{m}_{h t}=4,304 \mathrm{~m}_{e}$, where the subscripts 1 and $\mathrm{t}$ denote, respectively, the longitudinal (in the direction $\Gamma-\mathrm{X}$ ) and the transverse (in the direction $\mathrm{X}-\mathrm{W}$ ) components. This high anisotropy of masses of electron and hole ought to have resulted in the high anisotropy of the reduced $\left(1 / \mu=\mathrm{I} / \mathrm{m}_{e}+1 / \mathrm{m}_{h}\right)$ and the translation $\left(\mathrm{M}=\mathrm{m}_{e}+\mathrm{m}_{h}\right)$ masses of exciton. This, however, is not the case. Moreover, the study of Plekhanov and Altukhov [40] reveals that with a good degree of confidence one may assume that in the energy range $\mathrm{E} \leq 40 \mathrm{meV}$ the exciton band is isotropic and exhibits parabolic dispersion $\left(\mathrm{m}_{e}=0.04 \mathrm{~m}\right.$ and $\left.\mathrm{m}_{h}=0.15 \mathrm{~m}\right)$. As was shown below, the studies of exciton - phonon luminescence of free excitons and resonance Raman scattering of light in $\mathrm{LiH}$ crystals [22] reveal that the kinetic energy of excitons in these crystals is greater than $\mathrm{E}_{b}$ by an order of magnitude exactly because of the very small masses of electron and hole. The last one may indicate that in the metallic phase of hydrogen at the high pressure [22] we can expect the Dirac character of the electronic excitations [41]. We should add that isotope substitution will be very useful method for renormalization of the band - gap energy in graphene - future semiconducting material $\left[41^{a}\right]$.

\subsection{Semiconductors}

Semiconductors may be pure elements like Si (Ge), but may also be compounds (like LiH), for example $\mathrm{ZnS}, \mathrm{CdS}, \mathrm{SiC}, \mathrm{Cu}_{2} \mathrm{O}$ and GaAs. Their properties are strongly affected by the presence of impurities, defects or departures from exact stoichiometry. The properties of a perfect crystal of a pure element or perfectly stoichiometric compound are called intrinsic properties, whereas the influences of added impurities or defects give rise to extrinsic properties.

The electronic configuration of a Si atom is $1 \mathrm{~s}^{2} 2 \mathrm{~s}^{2} 2 \mathrm{p}^{6} 3 \mathrm{~s}^{2} 3 \mathrm{p}^{2}$ (see, e.g. [42]). When Si atoms form a crystal it can divide their electrons into core electrons and valence electrons as pointed in different textbooks $[43,44,45,46$, 17]. In crystalline Si $1 \mathrm{~s}, 2 \mathrm{~s}$ and $2 \mathrm{p}$ orbitals are completely occupied and form the core shells. The outer $3 \mathrm{~s}$ and 3 p shells are only partially filled. Electrons in these shells are called valence electrons because they are involved in bonding with neighboring Si atoms. The crystal structure of Si at ambient pressure is similar to that of diamond [28]. The tetrahedral arrangement of bonds between a Si atoms (see, e.g. Fig. 4.18 in [46]) and its four nearest neighbors can be understood if one of the electrons in the $3 \mathrm{~s}$ shell is promoted to the $3 \mathrm{p}$ shell so that the four valence electrons form hybridized $\mathrm{sp}^{3}$ orbitals. It is these valence electrons in the outermost shells of a $\mathrm{Si}$ atom that are nearly free. These electrons are not affected by the full nuclear charge as a result of screening of the nucleus by the filled core shells. In the core region the valence electron wave functions must be orthogonal to those of the core.

Consider further the formation of a $\mathrm{Si}$ dimer, i.e. a diatomic molecule. Just as in the formation of the $\mathrm{H}_{2}$ molecule, we expect the electron states to interact and form bonding and antibonding levels (see, Fig. 2). As is well - known, the separation into bonding and antibonding levels is the origin of the energy gap in the band structure of solid Si. According this very simple approximation, the lower four bands are filled and the upper four bands are empty [48]. One should note not worry about the detailed shapes of these bands but accept them as solutions of the Schrödinger equation in the assumed potential. Using the pseudopotential concept [48], the one electron Schrödinger equation have the next form

$$
\left[\frac{\mathrm{p}^{2}}{2 \mathrm{~m}}+\mathrm{V}\left(\overrightarrow{\mathrm{r}_{i}}\right)\right] \Psi_{k}\left(\overrightarrow{\mathrm{r}_{i}}\right)=\mathrm{E}_{k} \Psi_{k}\left(\overrightarrow{\mathrm{r}_{i}}\right)
$$



Figure 2. A schematic picture of bonding and antibonding levels in a silicon dimer

here $\Psi_{k}\left(\overrightarrow{r_{i}}\right)$ is the pseudo - wave - function and $\mathrm{V}\left(\overrightarrow{\mathrm{r}_{i}}\right)$ is pseudopotential. The bringing function is a good approximation to the true wave function outside the core region and therefore can be used to calculate the physical properties of the semiconductors which are dependent on the valence and conduction electrons only. The difference in energy between the highest occupied and lowest unoccupied state is indicated as $1.17 \mathrm{eV}$ at $0 \mathrm{~K}$. It is to be noted, however, that these limiting states lie at different points within the zone, and this minimum excitation of an electron from the valence to the conduction band demands that it be given crystal momentum equivalent to the difference in the $\overrightarrow{\mathrm{k}}$ vectors; this is readily obtained in thermal excitation by the crystal momentum available from the phonons (see, Fig. 3).

The energy gap characterizing an intrinsic semiconductor may be determined by optical absorption. At low temperatures there are very few carriers and the Drude contribution to the absoptivity is absent [46] in an intrinsic 




Figure 3. Electronic band structure of Si calculated by pseudopotential technique. The solid and the dotted lines represent calculations with a nonlocal and a local pseudopotential, respectively (after [48])

semiconductor. Semiconductors are therefore transparent to infrared radiation and become absorbing only when interband transitions are excited. We might therefore expect the band gap to fix the threshold for interband transitions and in this way be readily determined experiment [45]. This would be the case if the band gap were determined by filled and empty states with the same reduced value of $\overrightarrow{\mathrm{k}}$; the absorption of light would then cause a "vertical" transition and give rise to a sharp edge at $\hbar \omega=\mathrm{E}_{g}$. This is the case for many semiconductors such as CdS, GaAs etc.

In Si (and many other semiconductors), we have seen (Fig. 3) that excitation across the minimum separation of filled and empty states demands a large change in wave vector, and such a transition cannot be initiated by a photon unless it has access to a source of crystal momentum. We should repeat that it is the phonons that provide the required momentum (see, also Fig. 4). We write the conservation laws in the form

$$
\begin{aligned}
& \mathrm{E}_{f}-\mathrm{E}_{i}=\mathrm{h} \nu+\hbar \omega_{f}, \\
& \overrightarrow{\mathrm{k}_{f}}-\overrightarrow{\mathrm{k}_{i}}=0+\overrightarrow{\mathrm{q}},
\end{aligned}
$$



Figure 4. Schematic of two - step transition in the case of the indirect bandgap of material

here $\overrightarrow{\mathrm{q}}$ and $\omega_{f}$ apply to the phonon involved in the transition. Now $\mathrm{E}_{f}-\mathrm{E}_{i}=\mathrm{E}_{g}$ and it is clear that the inclusion of phonons produces an absorption edge at a somewhat lower energy, namely $\mathrm{E}_{g}$ - $\hbar \omega_{f}$. These indirect or phonon - assisted transitions produce only weak absorption compared with that associated with direct transitions (see, e.g. [49]).

\section{Excitons}

The properties of the electron and the hole are both described by the band structure within the one - electron approximation. in this section we shall go beyond this approximation and consider the effects of electron - electron interaction on the absorption spectra. To simplify the calculation we shall make the following assumptions. We shall include only the Coulombic part of the electron - electron interaction neglecting both exchange and correlation terms. Furthermore, the interaction between the excited electron in conduction band (see, Fig. 3) and those left behind in the now almost filled valence band will be replaced by an electron - hole interaction. Attraction between the electron and the hole causes their to be correlated and the resultant electron - hole pair is known as an exciton [50].

It has been more than eight decades since the introduction of quasiparticle exciton by Frenkel [50] and the extreme fertility of this idea has been demonstrated most powerfully. According to Frenkel the exciton is an electron excitation of one of the atoms (ions) of the crystal lattice, because of the translation symmetry, moves through the crystal in an electrically neutral formation. Since Frenkel the concept of an exciton has been developed 


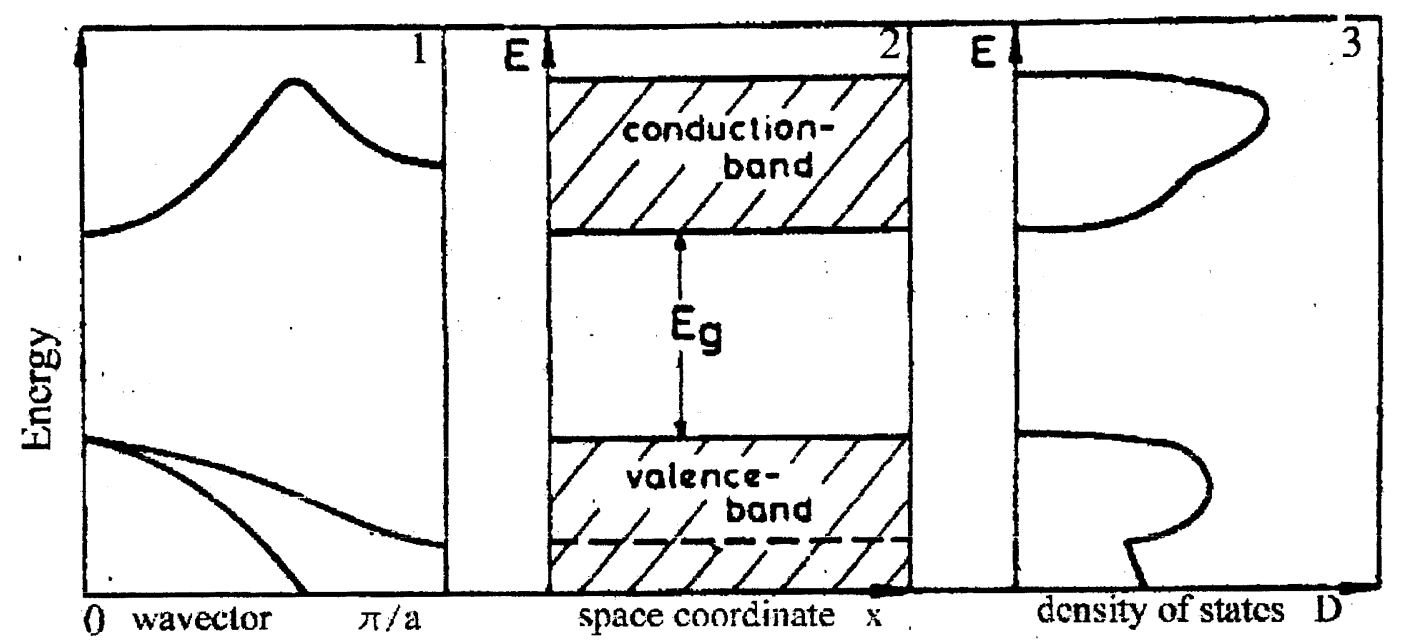

Figure 5. Various possibilities to present the band-structure of homogenous, undoped semiconductor (insulator). 1 - The dispersion relation i.e. the energy $\mathrm{E}$ as a function of the wavector $\vec{k}, 2$-The energy regions of allowed and forbidden states as a function of a space coordinate $\mathrm{x}$ and, 3 - the density of states (all curves are schematic ones)

in the papers of Peierls [51] and Slater and Schokley [52]. Problems concerning light absorption by solid state have been considered somewhat differently Wannier [53] and Mott [54]. According to the Wannier - Mott results the exciton is the state of an electron and hole bonded by the Coulomb force. The electron and hole in exciton state are spatially separated and their charges are screened. In the Frenkel papers the excitations localized on the lattice site were described thus, after the Wannier - Mott papers, the excitons became divided into the excitons on the Frenkel (small radius) excitons (for details see [55]) and the Wannier - Mott (large radius) excitons ( [65]). However, a description of the basic difference between these two models is absent [55 - 57]. The experimental discovery (see e.g. Gross [58]) of the Wannier - Mott exciton (see Fig.6) on the hydrogen-like absorption spectrum in the semiconducting crystals was the basis of a new subject - exciton physics (see also Agekyan [59]; Permogorov [60]). The influence of external perturbation (electrical and magnetic fields, uniaxial and hydrostatic deformation) on the optical spectra of the Wannier - Mott excitons (see e.g., Gross [58]) and their energetic characteristics (see also Cardona [61]) has been demonstrated repeatedly. These investigations permitted high-accuracy measurements not only the exciton binding energy but also of their translational mass, values of effective masses of the electron and hole, their $\mathrm{g}$ - factors etc. Moreover, the detailed account of the photon - exciton interaction has led to the concept of polaritons (Pekar [62). From the time of the experimental discovery of the Wannier - Mott exciton the problem concerning the interaction of excitons and the crystal lattice has persisted for more than four decades (Haken [63]; Haug and Koch [64]).

Below we briefly consider quantittively the effect of Coulomb attraction on the motion of electrons and holes (see, also [65]) in the vicinity of an $\mathrm{M}_{0}$ critical point of a direct bandgap semiconductor in three dimensions [61]. Further we shall assume the conduction band to be spherical with energy

$\mathrm{E}_{e}\left(\overrightarrow{\mathrm{k}_{e}}\right)=\mathrm{E}_{g}+\frac{\hbar^{2} \mathrm{k}_{e}^{2}}{2 \mathrm{~m}_{e}}$

where $\mathrm{E}_{g}$ is the band gap, and the corresponding hole energy to be given by

$\mathrm{E}_{h}\left(\overrightarrow{\mathrm{k}_{h}}\right)=\mathrm{E}_{g}+\frac{\hbar^{2} \mathrm{k}_{\mathrm{h}}^{2}}{2 \mathrm{~m}_{h}} \cdot \sum$

Let the Bloch function and the hole be represented by $\Psi_{\overrightarrow{k_{e}}}\left(\overrightarrow{r_{\mathrm{e}}}\right)$ and $\Psi_{\overrightarrow{k_{h}}}\left(\overrightarrow{r_{\mathrm{h}}}\right)$, respectively. As usually we assume that the Coulomb interaction between electron and hole is weak due to screening by the valence electrons so that the effective mass approximation is valid. We can write the exciton wavefunction $\Psi$ as linear combination of the electron and hole wavefunctions:

$$
\Psi=\sum_{\overrightarrow{\mathrm{k}_{\mathrm{e}}, \mathrm{k}_{\mathrm{h}}}} \mathrm{C}\left(\overrightarrow{\mathrm{k}_{e}}, \overrightarrow{\mathrm{k}_{h}}\right) \Psi_{\overrightarrow{k_{e}}}\left(\overrightarrow{r_{\mathrm{e}}}\right) \Psi_{\overrightarrow{k_{h}}}\left(\overrightarrow{r_{\mathrm{h}}}\right)
$$

Similar of the donor electron (see, e.g. [43, 49]), the electron and hole in an exciton are localized relative to their center of mass, so it is more convenient to express their wavefunctions in terms of Wannier functions rather than Bloch functions. In terms of the Wannier functions $a_{\overrightarrow{R_{e}}}\left(\overrightarrow{r_{e}}\right)$ and $a_{\overrightarrow{R_{h}}}\left(\overrightarrow{r_{h}}\right)$ for electron and hole, respectively, the exciton wavefunction can be written as

$$
\Psi\left(\overrightarrow{\mathrm{r}_{\mathrm{e}}}, \overrightarrow{\mathrm{r}_{\mathrm{h}}}\right)=\mathrm{N}^{-1 / 2} \sum_{\overrightarrow{\mathrm{R}_{\mathrm{e}}}, \overrightarrow{\mathrm{R}_{\mathrm{h}}}} \Phi\left(\overrightarrow{\mathrm{R}_{\mathrm{e}}}, \overrightarrow{\mathrm{R}_{\mathrm{h}}}\right) \mathrm{a}_{\overrightarrow{\mathrm{R}_{\mathrm{e}}}}\left(\overrightarrow{\mathrm{r}_{\mathrm{e}}}\right) \mathrm{a}_{\overrightarrow{\mathrm{R}_{\mathrm{h}}}}\left(\overrightarrow{\mathrm{r}_{\mathrm{h}}}\right)
$$

whhere $\Phi\left(\overrightarrow{R_{e}}, \overrightarrow{R_{h}}\right)$ is the exciton envelope wavefunction. The wave equation for $\Phi\left(\overrightarrow{R_{e}}, \overrightarrow{R_{h}}\right)$ is

$$
\left[-\left(\frac{\hbar^{2}}{2 \mathrm{~m}_{\mathrm{e}}}\right) \nabla_{\overrightarrow{\mathrm{R}_{\mathrm{e}}}}^{2}-\left(\frac{\hbar^{2}}{2 \mathrm{~m}_{\mathrm{h}}}\right) \nabla_{\overrightarrow{\mathrm{R}_{\mathrm{h}}}}^{2}-\frac{\mathrm{e}^{2}}{\varepsilon_{0}\left|\overrightarrow{\mathrm{R}_{\mathrm{e}}}-\overrightarrow{\mathrm{R}_{\mathrm{h}}}\right|}\right] \Phi\left(\overrightarrow{\mathrm{R}_{\mathrm{e}}}, \overrightarrow{\mathrm{R}_{\mathrm{h}}}\right)=\mathrm{E} \Phi\left(\overrightarrow{\mathrm{R}_{\mathrm{e}}}, \overrightarrow{\mathrm{R}_{\mathrm{h}}}\right)
$$

where $\varepsilon_{0}$ is the zero - frequency dielectric constant of material (insulators or semiconductors). Equation (13) can be solved in the same way as in the case of the hydrogen atom [66]. 




Figure 6. Discrete and continuous (hatched area) Wannier - Mott exciton energy spectrum taking into account its kinetic energy. The broken line connects to the dispersion of light in the medium

One expresses $\overrightarrow{R_{e}}$ and $\overrightarrow{R_{h}}$ in terms of two new coordinates: a center of mass coordinate $\vec{R}$ and a relative coordinate $\vec{r}$ defined by

$\overrightarrow{\mathrm{R}}=\frac{\overrightarrow{\mathrm{R}_{e}} m_{\mathrm{e}}+\overrightarrow{\mathrm{R}_{\mathrm{h}}} \mathrm{m}_{\mathrm{h}}}{\mathrm{m}_{\mathrm{e}}+\mathrm{m}_{\mathrm{h}}}$ and $\vec{r}=\overrightarrow{\mathrm{R}_{\mathrm{e}}}-\overrightarrow{\mathrm{R}_{\mathrm{h}}}$.

The equation of motion for the center of mass is now decoupled from that for the relative motion because the Coulomb interaction term does not involve $\overrightarrow{\mathrm{R}}$ (see, also [49, 65]). The two resultant equations are

$\left(-\frac{\hbar^{2}}{2 \mathrm{M}}\right) \nabla_{\overrightarrow{\mathrm{R}}}^{2} \Psi(\overrightarrow{\mathrm{R}})=\mathrm{E}_{R} \Psi(\overrightarrow{\mathrm{R}})$

$\left(-\frac{\hbar^{2}}{2 \mu} \nabla_{\overrightarrow{\mathrm{r}}}^{2}-\frac{\mathrm{e}^{2}}{\varepsilon_{0} r}\right) \phi(\overrightarrow{\mathrm{r}})=\mathrm{E}_{R} \phi(\overrightarrow{\mathrm{r}})$,

where $\mu$, the reduced mass of the exciton, is defined by

$\frac{1}{\mu}=\frac{1}{\mathrm{~m}_{\mathrm{e}}}+\frac{1}{\mathrm{~m}_{\mathrm{h}}}$.

The total energy of the exciton $\mathrm{E}$ is simply the sum of $\mathrm{E}_{\mathrm{R}}$ and $\mathrm{E}_{\mathrm{r}}$. The solution of (15) and (16) can be obtained readely. Equation (15) describes a free particle whose eigenfunction and energy are given by

$\Psi_{\mathrm{K}}(\overrightarrow{\mathrm{R}})=\mathrm{N}^{-1 / 2} \exp (\overrightarrow{\mathrm{K}} \cdot \overrightarrow{\mathrm{R}})$ and $\mathrm{E}_{R}=\frac{\hbar^{2} \mathrm{~K}^{2}}{2 \mathrm{M}}$.

$\mathrm{E}_{\mathrm{R}}$ represents the kinetic energy of the center of mass motion [65].

Equation (16) is similar to the equation describing the motion of the donor electron [49]. As in the hydrogen atom, its wavefunctions and energies can be described by three quantum numbers: a principal quantum number $\mathrm{n}$, the angular momentum quantum number $l$ and the magnetic quantum number $\mathrm{m}$. The wavefunction $\phi$ can be expressed in polar coordinates $(\mathrm{r}, \theta, \varphi)$ as

$\phi_{\mathrm{nlm}}(\overrightarrow{\mathrm{r}})=\mathrm{R}_{\mathrm{nl}}(\overrightarrow{\mathrm{r}}) \mathrm{Y}_{\operatorname{lm}}(\theta, \varphi)$,

where $\mathrm{R}_{\mathrm{nl}}(\overrightarrow{\mathrm{r}})$ and $\mathrm{Y}_{\operatorname{lm}}(\theta, \varphi)$ are, respectively, the associated Laguerre polynomials and the spherical harmonic functions. These functions are tabulated in many quantum mechanics textbooks and therefore will not reproduce here (see, e.g. [ 67]). For isotropic effective masses $\mathrm{E}_{\mathrm{r}}$ depends on $\mathrm{n}$ only and is given by

$\mathrm{E}_{\mathrm{r}}(n)=\mathrm{E}_{\mathrm{r}}(\infty)-\frac{\mathrm{R}^{*}}{\mathrm{n}^{2}}$,

where $E_{r}(\infty)$ is the minimum energy of the continuum states (see, Fig. 6), i.e. the energy gap $E_{g}$ and $R^{*}$ is the Rydberg constant for the exciton defined as [66]

$$
\mathrm{R}^{*}=\frac{\mu \mathrm{e}^{4}}{2 \hbar^{2} \varepsilon_{0}^{2}}=13.6 \cdot\left(\frac{\mu}{\mathrm{m} \varepsilon_{0}^{2}}\right) \mathrm{eV} .
$$

Combining the above result for the relative motion and the center of mass motion of the exciton, we obtain the following envelope functions and energies for the excitons: 


$$
\begin{aligned}
& \Phi_{\mathrm{nlm}}(\overrightarrow{\mathrm{R}}, \overrightarrow{\mathrm{r}})=(1 / \sqrt{\mathrm{N}}) \exp (\mathrm{i} \overrightarrow{\mathrm{K}} \cdot \overrightarrow{\mathrm{R}}) \mathrm{R}_{\mathrm{nl}}(\overrightarrow{\mathrm{r}}) \mathrm{Y}_{\operatorname{lm}}(\theta, \varphi), \\
& \mathrm{E}_{\mathrm{nlm}}=\mathrm{E}_{\mathrm{g}}+\frac{\hbar^{2} \mathrm{~K}^{2}}{2 \mathrm{M}}-\frac{\mathrm{R}^{*}}{\mathrm{n}^{2}}
\end{aligned}
$$

The energy spectrum of a Wannier - Mott excitons is shown in greater detail in Fig. 6.

The above model of excitons based on electrons and holes with spherically symmetric parabolic dispersion is useful for understanding exciton effects on optical spectra. However, it is not accurate enough for quantitative interpretation of experimental spectra in diamond and zinc - blende - type semiconductors. Of the various attempts to calculate excitonic effects based on realistic band structures, we shall mention the paper [68] (see, also [69]).

\section{Exciton - phonon interaction}

\subsection{Interaction between excitons and nonpolar optical phonons}

Foundations of the theory of exciton-phonon interaction were laid in the $1950 \mathrm{~s}$. The interaction between an electron and a nonpolar optical phonon in a crystal can be described simply in terms of a deformation potential (see e.g. [70]). The net effect of the lattice displacement on the electron is assumed to be a small shift in the electronic energy band of the crystal. The constant of proportionally between this energy shift and the lattice displacement is defined as the deformation potential. The exciton-phonon (deformational potential) Hamiltonian can therefore be expressed as [71]

$$
\mathrm{H}_{E P}=\sqrt{\frac{\hbar}{2 \mu \mathrm{N} \omega_{0}}} \frac{\left(W_{e}-\mathrm{W}_{h}\right)}{\mathrm{a}} \vec{a}_{k^{+}+\mathrm{q}}^{+} a_{k}\left[b_{q}+\mathrm{b}_{-q}^{+}\right]
$$

where $\mathrm{W}_{e}, W_{h}$ denote the deformation potentials of the electron and hole, respectively; $\mathrm{a}_{\vec{k}}^{+}$, $\mathrm{a}_{\vec{k}}-$ creation and annihilation operators of an exciton with wave vector $\vec{k} ; \mathrm{b}_{-\vec{q}}^{+}, b_{\vec{q}}$ - creation and annihilation operators of an optical phonon with momentum $\hbar \vec{q} ; \mu$ - reduced mass of the atoms in the unit cell; N - number of unit cells in the crystal; a - lattice constant of the crystal; and $\hbar \omega_{0}$ is the energy of the optical phonon (see also [61]).

\subsection{Polarization Interaction of Free Excitons with Phonon}

Apart from pioneering study in [34], interaction of excitons with longitudinal optical phonons was considered by many authors $[65,63,60,72,73]$. In ionic crystals there two main mechanisms of interaction of excitons with lattice vibrations. One - mechanism the short-range deformation interaction - is caused by modulation of the wave function of exciton by longitudinal acoustic vibrations. The magnitude of this interaction is characterized by the deformation potential (see above) The deformation interaction strongly affects the energy spectrum and dynamics of excitons of relatively small radius (e.g., the ground state of excitons in AHC and crystals of inert gases [65]. As the radius of exciton increases, this interaction becomes less important, since the wave vector of actual phonons is $\mathrm{q} \propto \mathrm{r}_{e x}^{-1}[74]$ where $\mathrm{r}_{e x}$ is the exciton radius, and the number of such phonons is proportional to $\mathrm{q}^{3}$.

The second mechanism - the polarization or Frohlich interaction [75] - is caused by the Coulombian interaction of the charge carriers forming the exciton with macroscopic field created by longitudinal optical oscillations (see e.g. $[76,73] ;)$. If the exciton radius is much greater than the lattice constant, then the exciton - phonon interaction may be regarded as the sum of independent interactions of electrons and holes with phonons (see [77]). The interaction operator of charge and mass $\mathrm{m}\left(\mathrm{m}_{e}\right.$ or $\left.\mathrm{m}_{h}\right)$ neglecting the dispersion of the latter is [75]:

$$
\mathrm{H}_{e l}=\sum_{\mathrm{q}} \mathrm{W}_{\vec{q}} \exp (\mathrm{iqr})\left(\mathrm{b}_{-\vec{q}}^{+}+\mathrm{b}_{\vec{q}}\right)
$$

where

$\mathrm{W}_{\vec{q}}=\frac{\hbar \omega_{L O}}{|\vec{q}|}\left[\frac{4 \pi q_{e, h}^{2}}{V}\right]^{1 / 2}$

$\mathrm{V}$ is the volume of the system and the $\vec{r}$ are the coordinates of the particles. In this expression we introduce the main parameters which determine the interaction of the electron (hole) with optical vibrations: the polaron "radius"

$$
\mathrm{r}_{e, h,}^{*}=\left(\frac{\hbar}{2} m_{e, h} \omega_{L O}\right)^{1 / 2}
$$

and the dimensionless Frohlich constant of interaction

$\mathrm{g}_{e, h}^{2}=\frac{1}{\alpha}\left(\frac{1}{\varepsilon_{\infty}}-\frac{1}{\varepsilon_{0}}\right) \frac{e^{2}}{\hbar \omega_{L O} r_{e, h}}$

The first of these quantities characterizes the size of the polarization region of the lattice by the extra charge, and the second describes the strength of the electron - phonon interaction (see also [65, 63]).

As follows from Eq. (26), the interaction operator $\mathrm{W}_{\vec{q}}$ does not depend on the mass of quasiparticle, and is the same for electrons and holes. Accordingly, the interaction Hamiltonian of Wannier - Mott excitons with optical phonons has the same form (25), the only difference that exp (iqr) is replaced with $\exp \left(\mathrm{iq}_{e} \mathrm{r}\right)$ - $\exp _{(\mathrm{iq}} \mathrm{r}$ ), where $\vec{r}_{e, h}$ are the coordinates of the electron (hole). In the center-of -mass system, the interaction operator becomes [71]:

$$
\mathrm{H}_{E X L}=\sum_{q} W_{\vec{q}}\left[\exp \left(i \mathrm{q}_{e} \mathrm{r}\right)-\exp \left(\mathrm{iq}_{h} \mathrm{r}\right)\right]\left(b_{-\vec{q}}^{+}+\mathrm{b}_{\vec{q}}\right) \text {. }
$$


Replacing $\mathrm{m}_{e, h}$ by the reduced mass $\mu$ by analogy with Eq. (25), we can define the characteristic size of the polarization region $\mathrm{r}_{e x}=\left[\left(r_{e}^{*}\right)^{2}+\left(r_{h}^{*}\right)^{2}\right]^{1 / 2}$ and the interaction constant $\mathrm{g}_{e x}^{2}$. Making use of the characteristics of the Wannier - Mott exciton $\left(\mathrm{r}_{e x}, \mathrm{E}_{b}\right)$, one can express the latter as

$$
\mathrm{g}_{e x}^{2}=\left(\frac{E_{b}}{\hbar \omega_{L O}}\right)^{1 / 2} \frac{\varepsilon_{0}}{\varepsilon_{\infty}}-1=\frac{r_{e x}^{*}}{r_{e x}}\left(\frac{\varepsilon_{0}}{\varepsilon_{\infty}}-1\right) \text {, }
$$

where $\mathrm{r}_{e x}=\mathrm{a}_{0}\left(\varepsilon \mathrm{m}_{0} / \mu\right)$, and $\mathrm{a}_{0}=\hbar^{2} / \mathrm{m}_{0} \mathrm{e}^{2}=0.53 \AA$ is the Bohr radius of hydrogen atom.

The scattering of excitons by LO phonons is determined by the magnitude and the wavevector dependence of the matrix element

$$
\mathrm{H}_{E X L}^{\lambda_{1} \lambda_{2}}=\left\langle\Psi_{\lambda_{1}}\left|H_{E X L}\right| \Psi_{\lambda_{2}}\right\rangle
$$

where $\Psi_{\lambda_{1}}$ and $\Psi_{\lambda_{2}}$ are the wavefunctions of the initial and final states of the exciton with the wavevectors $\mathrm{k}_{1}$ and $\mathrm{k}_{2}=\mathrm{k}_{1}+\vec{q}$. The properties of the matrix element of exciton - phonon scattering, as first noted in [78], depend crucially on the parities of the initial $\lambda_{1}$ and final $\lambda_{2}$ states. If the parity is the same (scattering occurs within the same band, like $1 \mathrm{~s}-1 \mathrm{~s}$ or $2 \mathrm{~s}-2 \mathrm{~s}$, etc., or in the case of interband scattering $1 \mathrm{~s}-2 \mathrm{~s}, 1 \mathrm{~s}-3 \mathrm{~s}$, etc.), this mechanism of exciton - phonon scattering is forbidden, because $\mathrm{H}_{E X L}^{\lambda_{1} \lambda_{2}} \rightarrow 0$ when $\mathrm{q} \rightarrow 0$. When excitons are scattered in a ground state band (1s - 1s) as shown in the paper of Ansel'm and Firsov, the matrix element has the form

$$
\mathrm{H}_{E X L}^{\lambda_{1} \lambda_{2}} \propto\left(\frac{g_{e x} \omega_{L O}}{r_{e x}}|q|\right)\left\{\left[1+\left(\frac{q_{e} r_{e x}}{2}\right)^{2}\right]^{-2}-\left[1+\left(\frac{q_{h} r_{e x}}{2}\right)^{2}\right]^{-2}\right\}
$$

When $\mathrm{q}$ is small (it is the small values that are of special importance; see below), the matrix element is proportional to

$$
\mathrm{H}_{E X L}^{\lambda_{1} \lambda_{2}} \propto\left(\frac{m_{h}-\mathrm{m}_{e}}{\mathrm{~m}_{h}+\mathrm{m}_{e}}\right) q r_{e x}
$$

As follows from Eq. (33), the Fröhlich mechanism of intraband scattering is absolutely forbidden when the effective masses are equal. This is because the centers of the distribution of masses and charges coincide at $\mathrm{m}_{e}=$ $\mathrm{m}_{h}$, so the polarization interactions of electrons and holes cancel out completely.

In the general case, the matrix element (32) arrives at maximum near $\mathrm{qr}_{e x} \sim 1$, and then falls off rapidly (Fig. 7). Similar behavior is displayed by the matrix elements of the scattering processes between of the same symmetry (is - js, ip - jp, etc.). In such cases, the matrix element attains its maximum at the value of the inverse radius $\left(\mathrm{r}_{n}\right)$ of the corresponding exciton state, that is,

$$
\mathrm{q}_{\max \sim} \frac{1}{n r_{n}}, r_{n}=\mathrm{nr}_{1} \text {. }
$$

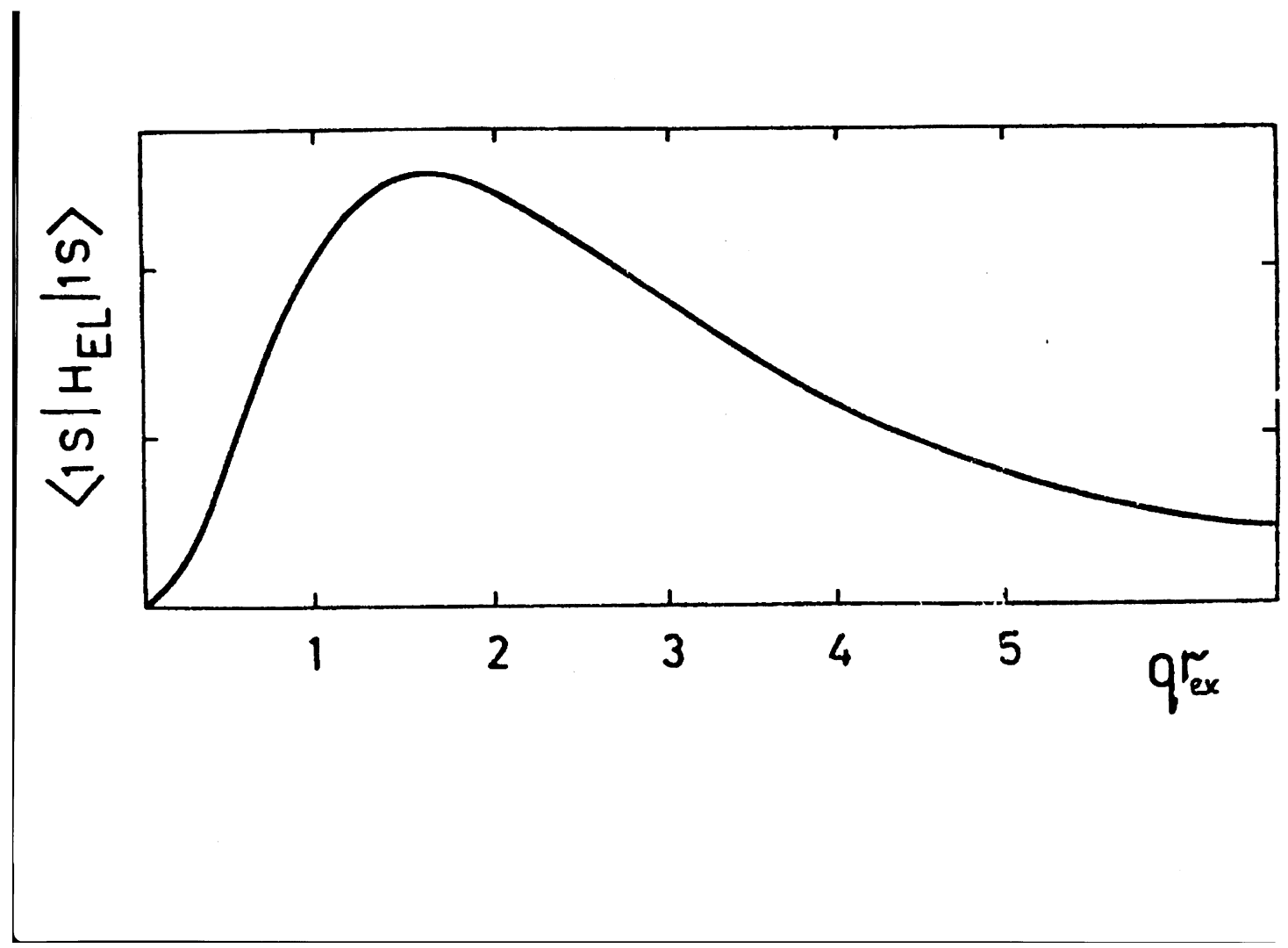

Figure 7. Dependence of matrix element of intraband scattering on $\mathrm{qr}_{e x}$ for the mass ratio $\mathrm{m}_{h} / \mathrm{m}_{c}=3.5$ (After [1])

According to the results of the paper by Bulyanitza, when scattering occurs between the bands of different symmetry (is - $\mathrm{kp}$ ) when $\mathrm{q} \rightarrow 0$ the matrix element tends to a constant (nonzero) value, and such processes are therefore allowed. As in our first case, however, the matrix element $\mathrm{H}_{E X L}^{\lambda_{1} \lambda_{2}}$ falls off rapidly as $\mathrm{q} \succ r_{e x}^{-1}$ increases. The dependence of matrix elements on q for some cases of allowed and forbidden scattering for CdS is discussed by Permogorov [60]. 
The behavior of matrix elements (32) is definitive for the structure and properties of luminescence spectra of free excitons and Raman scattering in the resonance region. The existing theory of exciton - phonon interaction describes the case of isotropic band with parabolic dispersion of exciton states. Its successful application to LiH (with the high anisotropy of the valence band, see Fig.1, once again testifies to the fact that the dispersion of the exciton band is indeed parabolic [79] in the range of low kinetic energies of exciton see Fig. 7 in [80]. Let us add that, according to Permogorov [60], the exciton band exhibits parabolic dispersion even for such anisotropic crystal as CdS, which is supported by the successful application of the theory of exciton-phonon interaction to the analysis of luminescence spectra and RRLS of free excitons in CdS crystals

Very recently Zheng and Matsuura [81] have published the paper devoted to electron - phonon interaction in a mixed crystals. They results we are briefly discussed. The total Hamiltonian of an electron - LO - phonon interaction system in a mixed crystal can be written as a sum of a free - electron Hamiltonian, the two - mode LO - phonon Hamiltonian, and the electron - phonon interaction Hamiltonian; it is given by

$\mathrm{H}=\frac{\hbar^{2}}{2 m} \nabla^{2}+\sum_{j=a, b} \hbar \omega_{L j} \hat{a}_{\vec{k} j}^{+} \hat{a}_{\vec{k} j}+\sum_{j=\mathrm{a}, \mathrm{b}} \sum_{\vec{k}} i \sqrt{\frac{e^{2} \hbar \omega_{L j}}{2 V \bar{\varepsilon}_{j} \varepsilon_{o} k^{2}}}\left(\hat{a}_{-\vec{k} j}^{+}+\hat{\mathrm{a}}_{\vec{k} j}\right) \mathrm{e}^{i \vec{k} \vec{r}}$,

where $\mathrm{m}$ is the conduction band mass of the electron. This problem is analogous to that of the polaron in polyatomic crystals [82]. Here they considered the first terms in Eq. (35) as the unperturbed Hamiltonian $\mathrm{H}_{0}$, and the last term as a perturbation $\mathrm{H}_{I}$. The energy of the system is calculated by the perturbation method. Let $\mid \vec{q}, 0>$ denote the zero - order wave function. The corresponding zero - order energy is $\mathrm{E}^{0}(\vec{q})=\hbar^{2} q^{2} / 2 \mathrm{~m}$. The first - order correction is obviously zero, that is, $\left\langle\vec{q}, 0\left|H_{I}\right| \vec{q}, 0\right\rangle=0$. The second - order correction can be written as

$\mathrm{E}^{\prime \prime}(\vec{q})=\sum_{j=\mathrm{a}, b} \sum_{\vec{k}} \frac{\left|\left\langle\vec{q}-\vec{k}, 1_{j \vec{k}}\left|H_{I}\right| \vec{q}, 0\right\rangle\right|^{2}}{E^{0}(\vec{q})-\mathrm{E}^{0}(\vec{q}-\vec{k})-\hbar \omega_{L j}}$

with

$\left|\left\langle\vec{q}-\vec{k}, l_{j \vec{k}}\left|H_{I}\right| \vec{q}, 0\right\rangle\right|^{2}=\frac{e^{2} \hbar \omega_{L j}}{2 V \bar{\varepsilon}_{j} \varepsilon_{o} k^{2}}$

Following the same procedure as Wang and Liang [83], Zheng and Matsuura replaced the sum over $\vec{k}$ by an integral in $\vec{k}$ space, then they obtain

$\mathrm{E}(\vec{q})=\frac{\hbar^{2} q^{2}}{2 m}+\sum_{j=\mathrm{a}, \mathrm{b}} \frac{e^{2} \hbar \omega_{L j}}{8 \pi^{2} \bar{\varepsilon}_{j} \varepsilon_{o}} \int_{o}^{\infty} \frac{d k \sin \theta d \theta}{\left(\hbar^{2} / 2 \mathrm{~m}\right)\left(2 k q \cos \theta-\mathrm{k}^{2}\right)-\hbar \omega_{L j}}$.

We restrict ourselves to the energy range $\hbar^{2} q^{2} / 2 \mathrm{~m}<\hbar \omega_{L j}$ and expand the integrand in Eq. (38) for small q. The polaron energy is given by

$$
\begin{aligned}
& \mathrm{E}(\vec{q}) \approx \frac{\hbar^{2} q^{2}}{2 m}-\sum_{j=\mathrm{a}, \mathrm{b}} \alpha_{j}\left[\hbar \omega_{L j}+\frac{\hbar^{2} q^{2}}{12 m}\right]=-\sum_{j=\mathrm{a}, \mathrm{b}} \alpha_{j} \hbar \omega_{L j}+\frac{\hbar^{2} q^{2}}{2 m^{*}} \\
& \text { with } \\
& \alpha_{j}=\frac{e^{2}}{8 \pi^{2} \bar{\varepsilon}_{j} \varepsilon_{o}}\left[\frac{2 m}{\hbar^{3} \omega_{L j}}\right], \mathrm{j}=\mathrm{a}, \mathrm{b} \\
& \mathrm{m}^{*}=\mathrm{m}\left[1-\frac{\alpha_{a}+\alpha_{b}}{6}\right]^{-1},
\end{aligned}
$$


Figure 8. Concentration dependence of the Fröhlich constants $\alpha_{a}$ (dotted line), $\alpha_{b}$ (dashed line) and $\alpha_{a}+\alpha_{b}$ (full line) of several mixed crystals (After [81])

where $\alpha_{a}$ and $\alpha_{b}$ are the Frohlich electron - phonon coupling constant of two LO - phonon modes in the mixed crystal, respectively; $\mathrm{m}^{*}$ is the polaron mass. The Frohlich coupling constant $\alpha_{a}$ and $\alpha_{b}$ and the total Frohlich coupling constant $\left(\alpha_{a}+\alpha_{b}\right)$ as functions of the composition $\mathrm{x}$ are shown in Fig. 8. The polaron energy shift $\alpha_{a} \hbar \omega_{L a}$ and $\alpha_{b} \hbar \omega_{L b}$ and the total polaron energy shift $\left(\alpha_{a} \hbar \omega_{L a}+\alpha_{b} \hbar \omega_{L b}\right)$ as function of the composition $\mathrm{x}$ are plotted in Fig. 9.

It is seen that although the concentration dependences of $\alpha_{a}$ and $\alpha_{b}$ display a strong nonlinear property, the total Frohlich coupling constant $\left(\alpha_{a}+\alpha_{b}\right)$ is almost exactly a straight line in figures. The polaron energy shifts of 

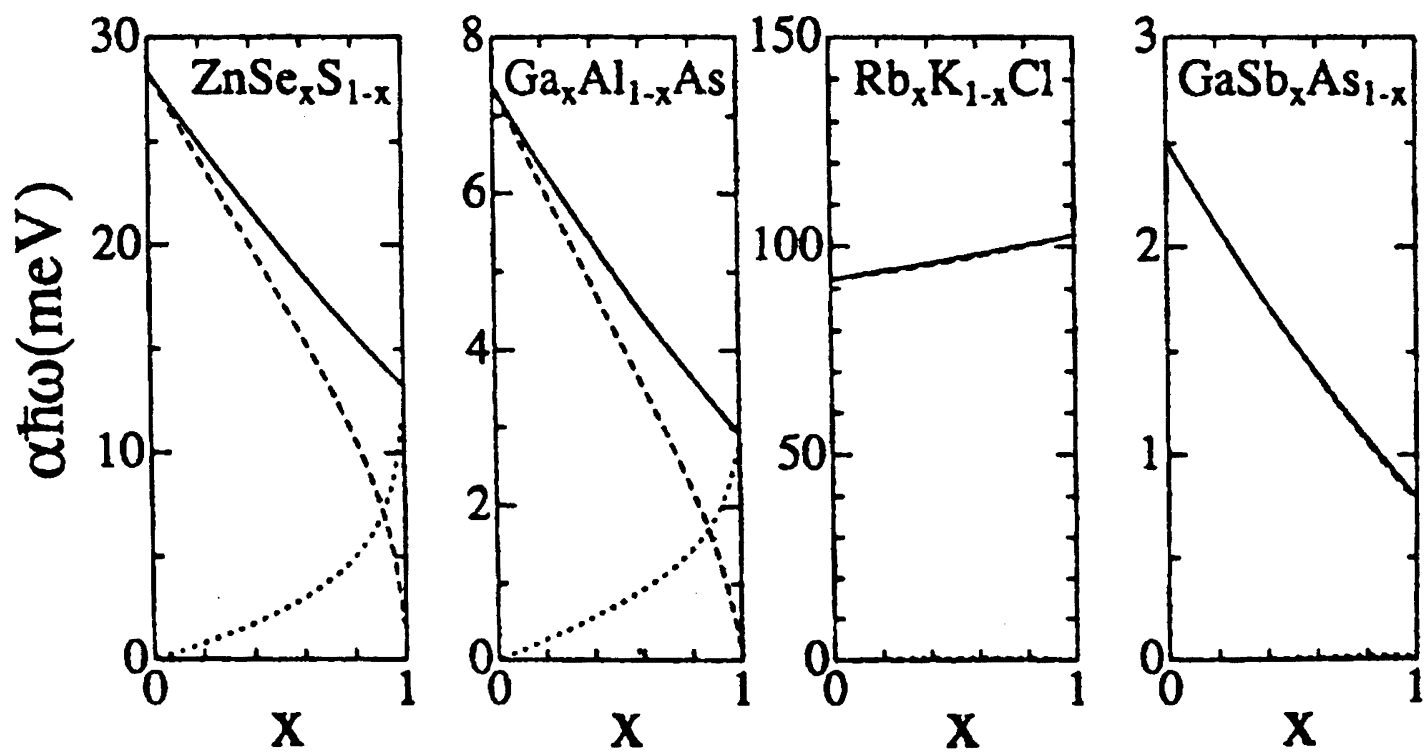

Figure 9. Concentration dependence of the polaron energy shift $\alpha_{a} \hbar \omega_{L a}$ (dotted line), $\alpha_{b} \hbar \omega_{L b}$ (dashed line), and the total polaron energy shift $\alpha_{a} \hbar \omega_{L a}+\alpha_{b} \hbar \omega_{L b}$ (full line) of several mixed crystals (After [81])

$\alpha_{a} \hbar \omega_{L a}$ and $\alpha_{b} \hbar \omega_{L b}$ also show a nonlinear feature, but the total polaron energy shift shows approximately a linear relation with the concentration $\mathrm{x}$. These theoretical results strongly support the linear interpolation approximation used by many experimenters to estimate the Frohlich constants and the polaron energy shifts of weak - coupling mixed crystals (see, e.g. [84, 85]). The results of Zheng and Matsuura are very different from theoretical results of Wang and Liang [83], where the expressions of the displacements of the two LO - phonon modes and the electron - phonon interaction Hamiltonian are not entirely correct.

In fact the sentence "the total Frohlich coupling constant $\left(\alpha_{a}+\alpha_{b}\right)$ is almost exactly linear function of the concentration $\mathrm{x}$ " implies that $\alpha_{a}(x)+\alpha_{b}(x) \simeq \mathrm{x} \alpha_{1}+(1-\mathrm{x}) \alpha_{2}$, where $\alpha_{1}$ and $\alpha_{2}$ are the Frohlich coupling constants of the two end members of the mixed crystal, respectively (see, however [39]). Theoretical calculations are too complex to see this result transparently. At the end we should noted that the theoretical results of Zheng and Matsuura are correct in the case of a weak - polar mixed crystal $\left(\alpha_{a}+\alpha_{b}\right) \leq 1$.

\section{Isotope affects on the electronic excitation}

\subsection{Semiconductors - small isotope effect}

In this section we will briefly discuss of the variation of the electronic gap $\left(\mathrm{E}_{g}\right)$ of semiconducting crystals with its isotopic composition. In the last time the whole raw of semiconducting crystals were grown. These crystals are diamond [86, 87], copper halides [88, 89], germanium [90, 91], silicon [92, 108 - 110], CdS [ 93, 93 ${ }^{a}$ and GaAs [88]. All numerated crystals show the dependence of the electronic gap on the isotope masses.

Before we complete the analysis of these results we should note that before these investigations, studies were carried out on the isotopic effect on exciton states for a whole range of crystals by Kreingol'd and coworkers (see, also [1]). First, the following are the classic crystals $\mathrm{Cu}_{2} \mathrm{O}[94]$ with the substitution ${ }^{16} \mathrm{O} \rightarrow{ }^{18} \mathrm{O}$ and ${ }^{63} \mathrm{Cu} \rightarrow{ }^{65} \mathrm{Cu}$. Moreover, there have been some detailed investigations of the isotopic effect on $\mathrm{ZnO}$ crystals (Fig. 10), where $\mathrm{E}_{g}$ was seen to increase by $55 \mathrm{~cm}^{-1}\left({ }^{16} \mathrm{O} \rightarrow{ }^{18} \mathrm{O}\right)$ and $12 \mathrm{~cm}^{-1}$ ( at $\left.{ }^{64} \mathrm{Zn} \rightarrow{ }^{68} \mathrm{Zn}\right)[95,97,98]$. In [96] it was shown that the substitution of a heavy ${ }^{34} \mathrm{~S}$ isotope for a light ${ }^{32} \mathrm{~S}$ isotope in CdS crystals resulted in a decrease in the exciton Rydberg constant $\left(\mathrm{E}_{b}\right)$, which was explained tentatively [99] by the contribution from the nearest electron energy bands, which however are absent in LiH crystals (see also [39]).

More detailed investigations of the exciton reflectance spectrum in CdS crystals were done by Zhang et al. [93]. Zhang et al. studied only the effects of Cd substitutions, and were able to explain the observed shifts in the band gap energies, together with the overall temperature dependence of the band gap energies in terms of a two-oscillator model provided that they interpreted the energy shifts of the bound excitons and $n=1$ polaritons as a function of average S mass reported as was noted above, earlier by Kreingol'd et al. [96] as shifts in the band gap energies. However, Kreingol'd et al. [99] had interpreted these shifts as resulting from isotopic shifts of the free exciton binding energies, and not the band gap energies, based on their observation of different energy shifts of features which they identified as the $\mathrm{n}=2$ free exciton states. The observations and interpretations, according Meyer at al. $\left[93^{a}\right]$, presented by Kreingol'd et al. [96] are difficult to understand, since on the one hand a significant band gap shift as a function of the S mass is expected [96], whereas it is difficult to understand the origin of the relatively huge change in the free exciton binding energies which they claimed. Very recently Meyer et al. [93 $\left.{ }^{a}\right]$ reexamine the optical spectra of CdS as function of average S mass, using samples grown with natural Cd and either natural S $\left(\sim 95 \%{ }^{32} \mathrm{~S}\right)$, or highly enriched $\left(99 \%{ }^{34} \mathrm{~S}\right)$. These author observed shifts of the bound excitons and the $\mathrm{n}=1$ free 


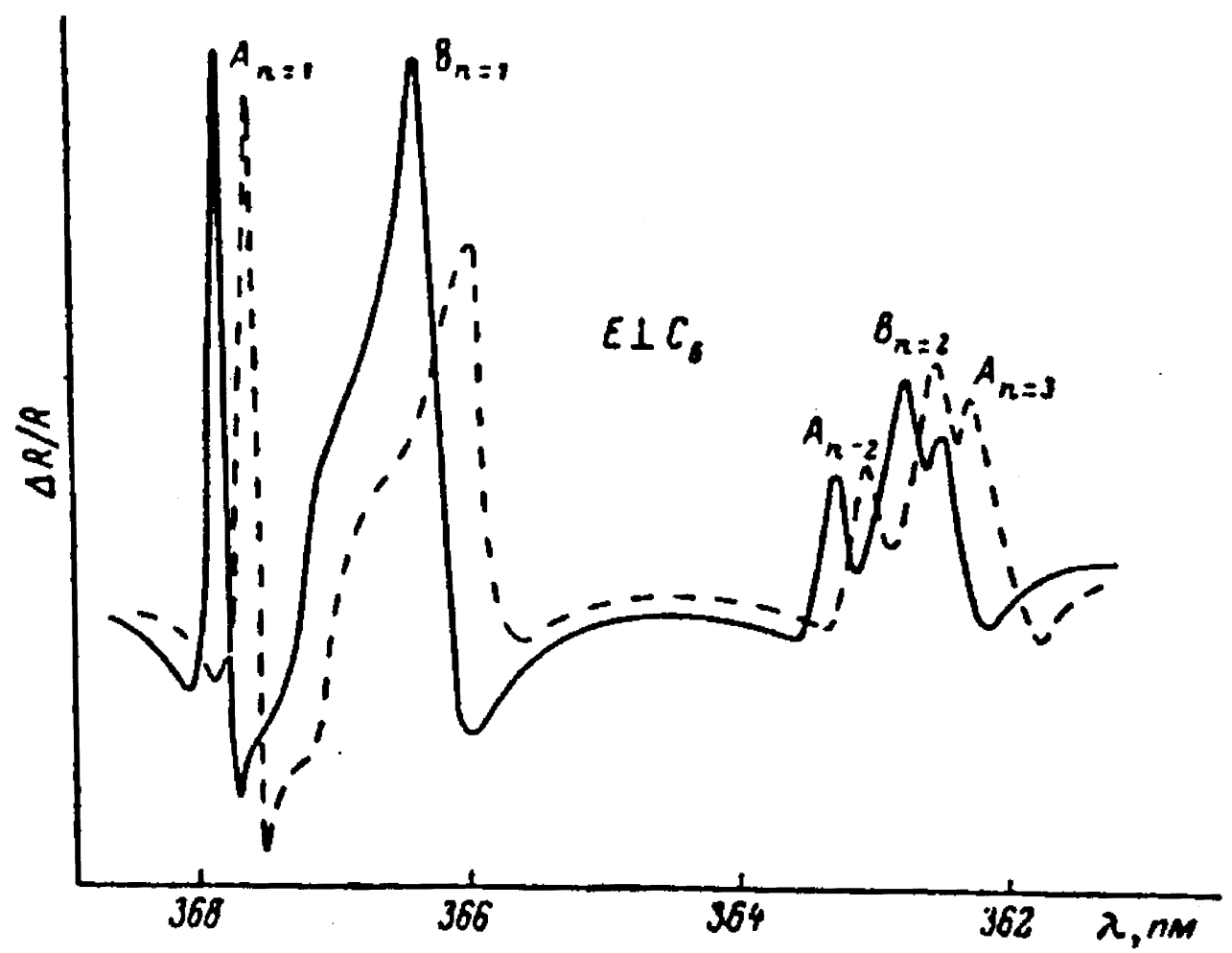

Figure 10. The reflection spectrum of ${ }^{65.4} \mathrm{Zn}^{16} \mathrm{O}$ crystal (solid line) and ${ }^{65.4} \mathrm{ZnO}_{0.67}^{16}{ }^{18} \mathrm{O}_{0.33}$ crystal dashed line)(After [98])

exciton edges consistent with those reported by Kreingol'd et al. [96], but, contrary to their results, Meyer et al. observed essentially identical shifts of the free exciton excited states, as seen in both reflection and luminescence spectroscopy. The reflectivity and photoluminescence spectra i polarized light $(\vec{E} \perp \vec{C})$ over the A and B exciton energy regions for the two samples depicted on the Fig. 10. For the $\vec{E} \perp \vec{C}$ polarization used in Fig.10 both A and $\mathrm{B}$ excitons have allowed transitions, and therefore reflectivity signatures. Fig. 11 also reveals both reflectivity signatures of the $n=2$ and 3 states of the A exciton as well that of the $n=2$ state of the B exciton (see, Fig. 12).

Meyer et al. summarized the energy differences $\Delta \mathrm{E}=\mathrm{E}\left(\mathrm{Cd}^{34} \mathrm{~S}\right)-\mathrm{E}\left(\mathrm{Cd}^{n a t} \mathrm{~S}\right)$, of a large number of bound exciton and free exciton transitions, measured using photoluminescence, absorption, and reflectivity spectroscopy, in $\mathrm{CdS}$ made from natural $\mathrm{S}\left(\mathrm{Cd}^{n a t} \mathrm{~S}, 95 \%{ }^{32} \mathrm{~S}\right)$ and from highly isotopically enriched ${ }^{34} \mathrm{~S}\left(\mathrm{Cd}^{34} \mathrm{~S}, 99 \%{ }^{34} \mathrm{~S}\right)(\mathrm{see}$ Table 2).

Table 2. The energy shifts of all of the transitions studied in $\left[93^{a}\right]$ are given in terms of the $\mathrm{Cd}^{34} \mathrm{~S}$ minus the $\mathrm{Cd}^{\text {nat }} \mathrm{S}$ energy, $\Delta \mathrm{E}$.

$\begin{array}{lll}\text { Transition } & \text { Method } & \Delta \mathrm{E}\left(\mathrm{cm}^{-1}\right) \\ \mathrm{I}_{2} & \mathrm{PL} & 10.6 \pm 0.1 \\ \mathrm{I}_{2}^{z} & \mathrm{PL} & 11.1 \pm 0.1 \\ \mathrm{I}_{2}^{a} & \mathrm{PL} & 10.6 \pm 0.1 \\ \mathrm{~A}_{n=1}\left(\Gamma_{6}\right) & \mathrm{A} \| & 10.8 \pm 0.2 \\ \mathrm{~A}_{n=1}\left(\Gamma_{5}^{L}\right) & \mathrm{PL} & 11.0 \pm 0.2 \\ \mathrm{~A}_{n=1}\left(\Gamma_{5}^{L}\right) & \mathrm{R} \perp & 10.9 \pm 0.2 \\ \mathrm{~A}_{n=2} & \mathrm{PL} \| & 11.3 \pm 0.4 \\ \mathrm{~A}_{n=2} & \mathrm{PL} \perp & 11.1 \pm 0.4 \\ \mathrm{~A}_{n=2} & \mathrm{R} \perp & 10.2 \pm 0.5 \\ \mathrm{~A}_{n=3} & \mathrm{PL} \| & 11.8 \pm 1.1 \\ \mathrm{~A}_{n=3} & \mathrm{PL} \perp & 10.9 \pm 0.6 \\ \mathrm{~A}_{n=3} & \mathrm{R} \perp & 10.7 \pm 0.6 \\ \mathrm{~B}_{n=1}\left(\Gamma_{1}\right) & \mathrm{R} \| & 10.9 \pm 0.3 \\ \mathrm{~B}_{n=1}\left(\Gamma_{5}^{L}+\Gamma_{5}^{T}\right) & \mathrm{R} \perp & 10.6 \pm 0.4 \\ \mathrm{~B}_{n=2} & \mathrm{R} \| & 9.4 \pm 1.2 \\ \mathrm{~B}_{n=2} & \mathrm{R} \perp & 9.8 \pm 1.2 \\ \mathrm{C}_{n=1}\left(\Gamma_{1}\right) & \mathrm{R} \| & 15 \pm 6 \\ \mathrm{C}_{n=1}\left(\Gamma_{5}\right) & \mathrm{R} \perp & 14 \pm 5\end{array}$

The methods used were photoluminescence spectroscopy $\left({ }^{*} \mathrm{PL}\right)$ and reflection spectroscopy (R). For measurements made using polarized light, the $\|$ or $\perp$ specifies the orientation of the $\mathbf{E}$ vector vs the $\mathrm{c}$ axis.

As we can see, all of the observed shifts are consistent with a single value, $10.8 \pm 0.2 \mathrm{~cm}^{-1}$. Several of the donor bound exciton photoluminescence transitions, which in paper [68] can be measured with high accuracy, reveal shifts 

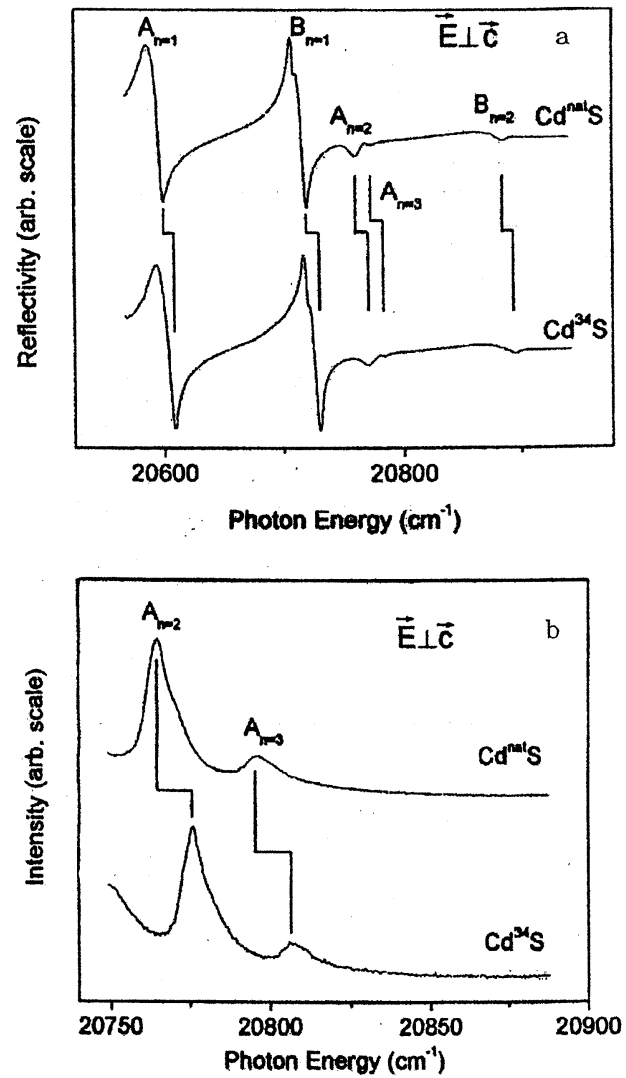

Figure 11. Photoreflectance of (a) ${ }^{112} \mathrm{CdS}$ and (b) ${ }^{n a t} \mathrm{CdS}$ at $6 \mathrm{~K}$. The assignment of the spectral features to various components of the series of A and B excitons is indicated. (After Zhang et al $\left[93^{a}\right]$ )


Figure 12. Dependence of the ground-state energy of the $\Gamma_{5}^{L}(\mathrm{~A})(\mathrm{a})$ and $\Gamma_{5}^{L}(\mathrm{~B})$ excitons (b) on the isotopic mass of Cd obtained from photoreflectance measurements at $6 \mathrm{~K}$. The solid lines represent the best fit with a straight line (After [93]) 
which differ from each other by more than the relevant uncertainties, although all agree with the $10.8 \pm 0.2 \mathrm{~cm}^{-1}$ average shift. These small differences in the shift energies for donor bound exciton transitions may reflect a small isotopic dependence of the donor binding energy in CdS. This value of $10.8 \pm 0.2 \mathrm{~cm}^{-1}$ shift agrees well with the value of $11.8 \mathrm{~cm}^{-1}$ reported early by Kreingol'd et al. [96] for the $\mathrm{B}_{n=1}$ transition, particularly when one takes into account the fact that enriched ${ }^{32} \mathrm{~S}$ was used in that earlier study, whereas Meyer et al. have used natural $\mathrm{S}$ in place of an isotopically enriched $\mathrm{Cd}^{32} \mathrm{~S}$.

Authors $\left[93^{a}\right]$ conclude that all of the observed shifts (see Table 2) arise predominantly from an isotopic dependence of the band gap energies, and that the contribution from any isotopic dependence of the free exciton binding energies is much smaller. On the basis of the observed temperature dependencies of the excitonic transitions energies, together with a simple two-oscillator model, Zhang et al. $\left[93^{a}\right]$ earlier calculated such a difference, predicting a shift with the $\mathrm{S}$ isotopic mass of $950 \mu \mathrm{eV} / \mathrm{amu}$ for the A exciton and $724 \mu \mathrm{eV} / \mathrm{amu}$ for the B exciton. Reflectivity and photoluminescence study of ${ }^{n a t} \mathrm{Cd}^{32} \mathrm{~S}$ and ${ }^{n a t} \mathrm{Cd}^{34} \mathrm{~S}$ performed by Kreingol'd et al. [99] shows that for anion isotope substitution the ground state $(\mathrm{n}=1)$ energies of both $\mathrm{A}$ and $\mathrm{B}$ excitons have a positive energy shifts with rate of $\partial \mathrm{E} / \partial \mathrm{M}_{S}=740 \mu \mathrm{eV} / \mathrm{amu}$. Results of Meyer et al. $\left[93^{a}\right]$ are consistent with a shift of $\sim 710$ $\mu \mathrm{eV} / \mathrm{amu}$ for both A and B excitons. Finally, it is interesting to note that the shift of the exciton energies with $\mathrm{Cd}$ mass is $56 \mu \mathrm{eV} / \mathrm{amu}$ [96], an order of magnitude less than found for the $\mathrm{S}$ mass.

The electronic band structures of semiconductors with a diamond or zinc-blende crystal lattice have a degenerate valence-band maximum for light and heavy holes at the center of the Brillouin zone $(\vec{k}=0, \Gamma$ point $)$ as well as a split-off valence band with its maximum at the same location in $\mathrm{k}$ space. Three conduction-band minima are observed at the high-symmetry points $\Gamma$, L, and X. The lowest energy conduction-band minimum occurs in Ge at the $\mathrm{L}$ point and in $\mathrm{Si}$ near the $\mathrm{X}$ point, forming indirect band gaps in these two elemental semiconductors. The width and the character (direct or indirect) of this lowest energy gap is of paramount importance for a large number of semiconductors properties and in turn for all semiconductor devices. Because of this great significance there exists a strong interest in all effects which influence the band structure.



Figure 13. Transmission spectra of ${ }^{76} \mathrm{Ge}(1)$ and ${ }^{72} \mathrm{Ge}(2)$ in the vicinity of the indirect excitons transitions at $1.7 \mathrm{~K}$. (After [90])

The isotopic composition affects the band gaps through the electron-phonon coupling and through the change of volume with isotopic mass. Several groups have conducted low-temperature studies of the direct and indirect band gaps of natural and isotopically controlled Ge and Si single crystals. For the first time Agekyan et al. [90] used photoluminescence, infrared absorption, and Raman spectroscopy with a Ge crystals of natural composition and a crystals with $85 \%{ }^{76} \mathrm{Ge}$ and $15 \%{ }^{74} \mathrm{Ge}$. They found an indirect (see Fig. 13) band-gap change $\Delta \mathrm{E}_{g}=0.9$ $\mathrm{meV}$ and a direct band-gap change $\Delta \mathrm{E}_{g}=1.25 \mathrm{meV}$ with an error of $\pm 0.05 \mathrm{meV}$. Etchegoin et al. [100] and Davies et al. [101] reported photoluminescence studies of natural and several highly enriched, high quality single crystals of Ge. Measurement of the energies of impurity-bound excitons permits by Davies et al. the direct determination of band-gap shifts with the crystal isotope mass because the radiative recombination does not require phonon participation. Fig. 14 shows the no-phonon energies of excitons $\mathrm{E}_{N P}$ bound to $\mathrm{P}$ and $\mathrm{Cu}$ in several isotopically controlled crystals. As may be expected from the very large Bohr orbit of the excitons (see [101] and reference therein), their binding energy only depends on the average isotope mass and not on the isotopic disorder (see, 
however [102]). The rate of band-gap energy change with isotope mass as determined by Davies et al. [101] is $\mathrm{dE}_{I G} / \mathrm{dA}=\mathrm{dE}_{N P} / \mathrm{dA}=0.35 \pm 0.02 \mathrm{meV} / \mathrm{amu}$.

Etchegoin et al. [100] obtained a very similar value.

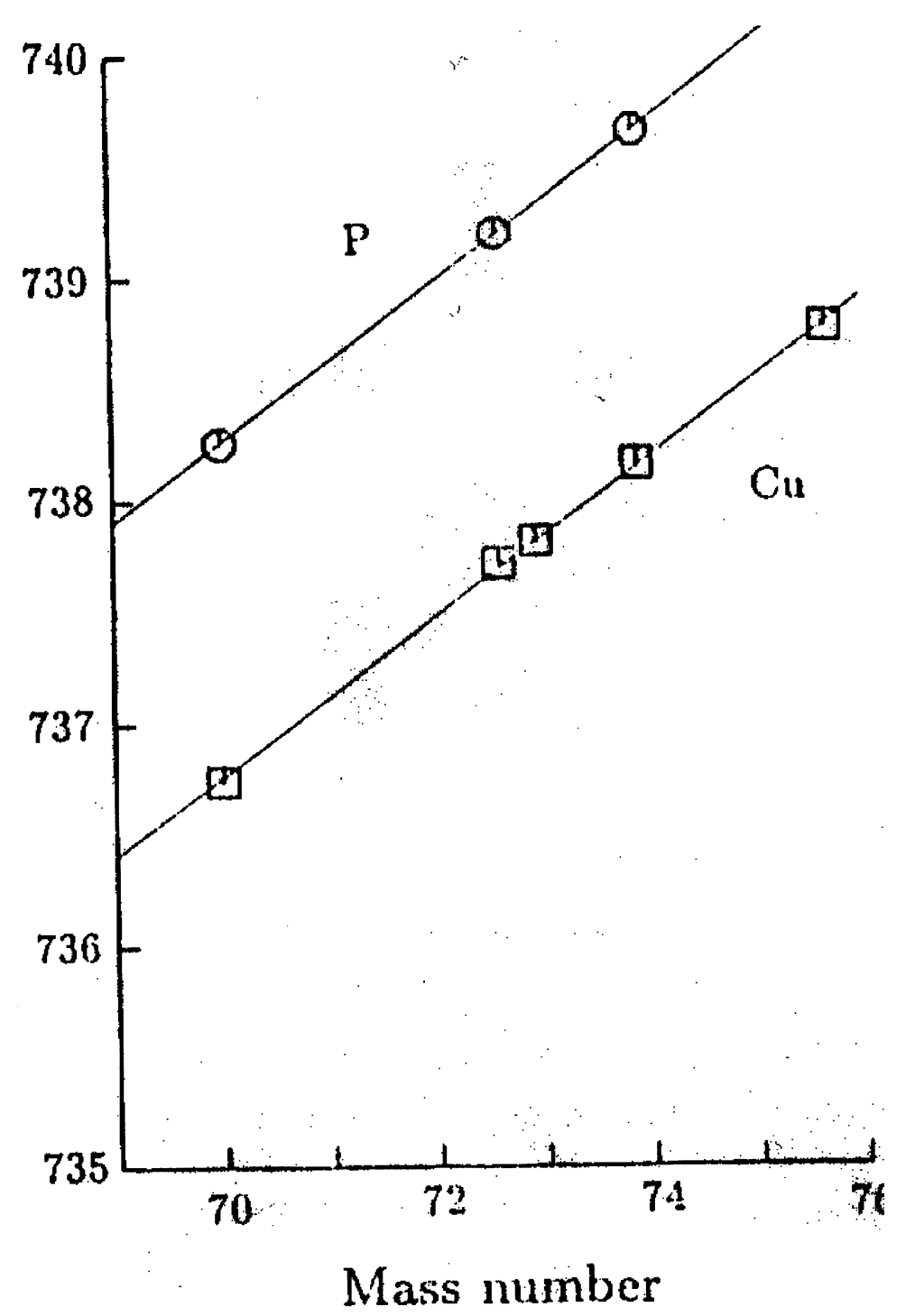

Figure 14. Energies of the no-phonon lines of excitons bound to $\mathrm{Cu}$ acceptors (squares) and P donors (circles) (After [101])

The contribution to the band-gap shift originating in the volume change can be estimated using the results of Buschert et al. [103] for lattice-constant change with isotope mass and the published dependence of $\mathrm{E}_{I G}$ with volume [104]. They found

$\left(\mathrm{dE}_{I G} / \mathrm{dA}\right)_{v o l}=0.132 \mathrm{meV} / \mathrm{amu}$.

This is the smaller contribution to the experimentally determined energy-gap change with isotope mass. It is in reasonable agreement with the earlier estimates of Agekyan et al.

The main contribution to $\mathrm{dE} / \mathrm{dA}$ can be directly related to the change of the energy gap with temperature. This change is described by structure factors which contain electron-phonon interaction terms (Debye-Waller factors) and self-energy terms. For practical calculations these terms are expanded in a power series of the atomic displacements. The leading terms are proportional to the mean-square displacements $\left\langle u^{2}\right\rangle$ of each atom. Describing the lattice atoms in terms of harmonic oscillators, one find

$\left\langle\mathrm{u}^{2}\right\rangle=\hbar\left(\frac{1}{2}+\mathrm{n}\right) / M \omega$.

With increasing temperature both $\mathrm{n}$ and $\left\langle u^{2}\right\rangle$ increase, leading to the observed reduction of the energy gap. At low temperature $\mathrm{n}=0$ and we only deal with the zero-point oscillation. Combining the dependence of $\omega$ on $\mathrm{M}$ with the above equation one find

$\left\langle\mathrm{u}^{2}\right\rangle \propto 1 / \sqrt{M}$

Zollner et al. [105] have performed a numerical calculation of the electronic bands using an empirical pseudopotential method including the necessary lattice dynamics. They found for $\mathrm{Ge}\left(\mathrm{dE}_{I G} / \mathrm{dA}\right)_{e-p}=0.41 \mathrm{meV}$. The total calculated shift of the indirect band-gap energy with isotope mass adds up to $\left(\mathrm{dE}_{I G} / \mathrm{dA}\right)_{\text {total }}=0.48 \mathrm{meV}$. This result compares favorably with the experimental values stated above by Davies et al. and by Etchegoin et al. who reported $\left(\mathrm{dE}_{I G} / \mathrm{dA}\right)_{\text {total }}=0.37 \pm 0.01 \mathrm{meV} / \mathrm{amu}$ (see also Fig 15). 




Figure 15. Atomic mass dependence of the indirect gap $\mathrm{E}_{g}$ of $\mathrm{Ge}$ at $\mathrm{T}=6 \mathrm{~K}$ (After [107])

Measurements of the direct band gap at the $\Gamma$ point $(\vec{k}=0)$ in the Brillouin zone have also been performed. Though the direct band gap is technologically less important than the minimum indirect band gap, determining the dependence of this gap on isotope mass is of the same fundamental significance as the indirect band-gap studies. Davies et al. [101] used low-temperature optical-absorption measurements of very thin samples of Ge single crystals with natural composition and three different, highly enriched isotopes. They found

$\mathrm{dE} / \mathrm{dA}=0.49 \pm 0.03 \mathrm{meV} / \mathrm{amu}$

for the temperature extrapolated to zero. Parks et al. [107] have used piezo- and photomodulated reflectivity spectra of four monoisotopic and one natural Ge crystals. These techniques do not require the extreme sample thinning which is necessary for optical-absorption measurements and the derivative nature of the spectra emphasizes the small changes. The excellent signal-to-noise ratio and the superb spectral resolution allowed a very accurate determination of the dependence of $\mathrm{E}_{D G}$ on isotopic mass (Fig. 16). At very low temperatures an inverse squareroot dependence accurately describes band-gap dependence:

$\mathrm{E}_{D G}=\mathrm{E}_{D G}^{\infty}+\frac{C}{\sqrt{M}}(47)$.

A fit through five data points yields $\mathrm{E}_{D G}^{\infty}=959 \mathrm{meV}$ and $\mathrm{C}=-606 \mathrm{meV} / \mathrm{amu}^{1 / 2}$. Written as a linear dependence for the small range of isotopic masses, Parks et al. find $\mathrm{dE}_{D G} / \mathrm{dA}=0.49 \mathrm{meV} / \mathrm{amu}$, in perfect agreement with the results of Davies et al. [101]. Parks et al. also determined the isotope mass dependence of the sum of the direct gap and the split-off valence band $\left(\Delta_{0}\right)$ and find $\mathrm{d}\left(\mathrm{E}_{D G}+\Delta_{0}\right) / \mathrm{dA}=0.74 \mathrm{meV} / \mathrm{amu}$. The experimental results can be compared to the Zollner et al. [105] calculations which are found to be of the correct order of magnitude. The theoretical estimates for the contributions of the linear isotope shifts of the minimum, indirect gaps which are caused by electron-phonon interaction, are too large by a factor of $\sim 1.7$ and for the smallest direct gap they are too large by a factor $\sim 3.2$.

Substitution of ${ }^{70} \mathrm{Ga}$ on the ${ }^{76} \mathrm{Ga}$ increases the band gap in GaAs [88] on $10.5 \mathrm{~cm}^{-1}$ (see, also Table 3). The interested results were communicated in papers of Cardona and coworkers [89], where it was studied the dependence of $\mathrm{E}_{g}$ on the isotope effect in $\mathrm{CuCl}$ crystals. When the ${ }^{64} \mathrm{Cu}$ on the ${ }^{65} \mathrm{Cu}$ is substituted the value of $\mathrm{E}_{g}$ in $\mathrm{CuCl}$ crystals decreased on $1.24 \mathrm{~cm}^{-1}$, e.g. the isotope effect on the electronic excitation has an opposite sign.

The present knowledge of the electronic band structure of Si stems from experimental observation of electronic transitions in transmission, reflectivity, or cyclotron resonance, on the one hand, and theoretical calculations, e.g. those based on pseudopotential or $\vec{k} \cdot \vec{p}$ methods (for details see [17] and references therein). In this manner it has been established that the fundamental, indirect band gap of $\mathrm{Si}$ occurs between the $\Gamma_{8}^{+}$valence band maximum and the $\Delta_{0}$ conduction band minima along (100).

Recently, Lastras-Martinez et al. [92] performed ellipsometric measurements on isotopically enriched ${ }^{28} \mathrm{Si}$ and ${ }^{30} \mathrm{Si}$ and deduced the isotopic dependence of $\mathrm{E}_{1}$ from the analysis of the data in reciprocal (Fourier inverse) space. However, these measurements did not resolve the nearly degenerate $\mathrm{E}_{0}{ }_{0}$ and $\mathrm{E}_{1}$ transitions and the isotopic shift was assigned solely to the stronger $\mathrm{E}_{1}$ transitions (see, however, Fig. 17). We should add that in papers [108] very recently was studied the dependence of indirect band gap in $\mathrm{Si}$ on the isotopic mass. Photoluminescence and 



Figure 16. Isotopic mass (in amu) dependence of the (a) $\mathrm{E}_{0}$ and (b) $\mathrm{E}_{0}+\Delta_{0}$ direct energy gaps obtained from photomodulated reflectivity measurements at $\mathrm{T}=6 \mathrm{~K}$. The curves are the best to relation $\mathrm{E}_{0}=\mathrm{E}_{0}^{\infty}+\frac{C}{\sqrt{M}}$, where $\mathrm{M}$ is the atomic mass and $\mathrm{E}_{0}^{\infty}$ is the energy gap at $\mathrm{M}=\infty$. The fitting yields $\mathrm{E}_{0}^{\infty}=959 \mathrm{meV}$ and $\mathrm{C}=-606 \mathrm{meV} / \mathrm{amu}$ (After Parks et al [107])

wavelength-modulated transmission spectra displaying phonon assisted indirect excitonic transitions in isotopically enriched ${ }^{28} \mathrm{Si},{ }^{29} \mathrm{Si},{ }^{30} \mathrm{Si}$ as well as in natural $\mathrm{Si}$ have yielded the isotopic gap $\mathrm{E}_{g x}$ which equals $1213.8 \pm 1.2 \mathrm{meV}$. This is purely electronic value in the absence of electron-phonon interaction and volume changes associated with anharmonicity (for details see [92] and below).

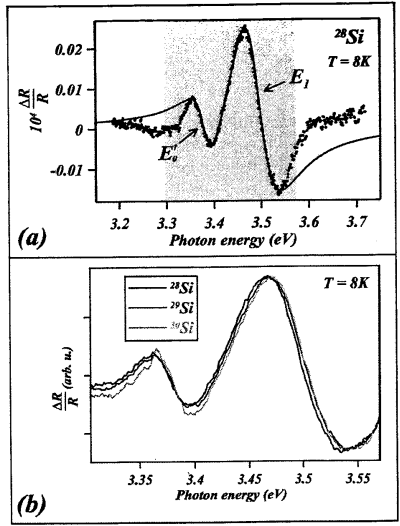

Figure 17. a -Signatures of the $\mathrm{E}_{0}$ ' and $\mathrm{E}_{1}$ excitonic band gaps of ${ }^{28} \mathrm{Si}$ observed (dots) in photomodulated reflectivity. The solid line is a theoretical fit using the excitonic line shape. b - Photomodulated reflectivity spectra of isotopically enriched Si exhibiting isotopic shifts of the $\mathrm{E}_{0}$ ' and $\mathrm{E}_{1}$ gaps (after [108])

Returning to Fig. 17, we can see that the spectrum contains two characteric signatures, attributed to the excitonic transitions across the $\mathrm{E}_{0}{ }_{0}$ and $\mathrm{E}_{1}$ gaps. Isotopic dependence of the $\mathrm{E}_{0}{ }_{0}$ and $\mathrm{E}_{1}$ is displayed in Fig. 17, where the photomodulated reflectivity spectra of ${ }^{28} \mathrm{Si},{ }^{29} \mathrm{Si}$, and ${ }^{30} \mathrm{Si}$ are shown for the spectral range $3.3 \leq \mathrm{E}$ $\leq 3.58 \mathrm{eV}$. The $\mathrm{E}_{0}^{\prime}$ and $\mathrm{E}_{1}$ excitonic band gaps determined in paper [108] from the line-shape analysis. Linear least-squares fit yielded the corresponding isotopic dependences $\mathrm{E}_{0}^{\prime}=\left(3.4468-0.3378 \mathrm{M}^{-1 / 2}\right) \mathrm{eV}$ and $\mathrm{E}_{1}=(3.6120$ - $\left.0.6821 \mathrm{M}^{-1 / 2}\right) \mathrm{eV}$. In concluding, we should note that the spin-orbit interaction depends in Ge in contrast to that in $\mathrm{Si}[92]$.

As is well known ago, the fundamental energy gap in silicon, germanium, and diamond is indirect (see, e.g. Fig. 3). While the conduction band minima in Si and diamond are located at the $\Delta$ point along $<100>$, with $\Delta_{6}$ symmetry, those of germanium with $\mathrm{L}_{6}^{+}$symmetry occur at the $<111>$ zone boundaries [92]. The onset of the absorption edge corresponds to optical transition from the $\Gamma_{8}^{+}$valence band maximum to the $\mathrm{L}_{6}^{+}$conduction band minima in Ge, and the $\Delta_{6}$ in $\mathrm{Si}$ and diamond; for wavector conservation, these indirect transitions require the emission or absorption of the relevant phonons. In $\mathrm{Si}$ and $\mathrm{C}$, transverse acoustic (TA), longitudinal acoustic (LA), transverse optic (TO), or longitudinal optic (LO) phonons of $\Delta$ symmetry must be simultaneously emitted or absorbed. In Ge (see, also above), the wavector conserving phonons are TA, LA, TO or LO phonons with L symmetry. At low temperatures, these indirect transitions are assisted by phonon emission. In this case we should 
expect at low temperatures four excitonic derivative signatures at photon energies $\mathrm{E}_{g x}+\hbar \omega_{\vec{q}},{ }_{j}$ in modulated transmission experiments and in photoluminescence at the photon energies $\mathrm{E}_{g x}-\hbar \omega \vec{q},{ }_{j}$. Here $\mathrm{E}_{g x}$ is the excitonic band gap and $\mathrm{j}$ corresponds to a wave vector preserving phonon (see, also formulae (8)). In Fig. $18^{a}$ the photoluminescence and wavelength - modulated spectra of ${ }^{30} \mathrm{Si} \mathrm{M}=2.81 \mathrm{amu}$ ) are displayed; the labels $\mathrm{n}=1$ and 2 designate the ground and the first excited states of the indirect TA and and TO excitons. From the energies of the photoluminescence and wavelength-modulated excitonic signatures in all isotopic specimens (see [92]) cited authors deduce $\mathrm{E}_{g x}$ as well as the energies of the participating TO, LO and TA phonons, shown in Fig. 12 - B as function of $\mathrm{M}^{-1 / 2}$. The excitonic band gap data are fitted well with expression $\mathrm{E}_{g x}(\mathrm{M})=\mathrm{E}_{g x}(\infty)-\mathrm{CM}^{-1 / 2}$, yielding $\mathrm{E}_{g x}(\infty)=(1213.8 \pm 1.2) \mathrm{meV}$ and $\mathrm{C}=(313.7 \pm 5.3) \mathrm{meV} / \mathrm{amu}$. A linear fit in $\mathrm{M}$ can be made over small range of available masses (see, Fig. $\left.18^{b}\right)$ with a slope $\left(\partial \mathrm{E}_{g x} / \partial \mathrm{M}\right)_{P, T} 1.01 \pm 0.04 \mathrm{meV} / \mathrm{amu}$, which agrees with the results of bound exciton photoluminescence of Karaiskaj et al. [113]. The experiments in papers [108 - 110] also indicate that separation of the $n=2$ and $n=1$ excitons is isotope mass independent, implying, according these authors, the excitonic binding energy is independent on isotope mass within experimental error. In concluding this part we should note that recent high - resolution spectroscopic studies of excitonic and impurity transition in high - quality samples of isotopically enriched Si have discovered the broadening of bound exciton emission (absorption) lines connected with isotope - induced disorder as well as the depend of their binding energy on the isotope mass [72, 108 - 110]. The last effect was early observed on the bound excitons in diamond [112, 17], and earlier on the free excitons [114] in $\mathrm{LiH}_{x} \mathrm{D}_{1-x}$ mixed crystals (see, e.g. [115] and references therein).
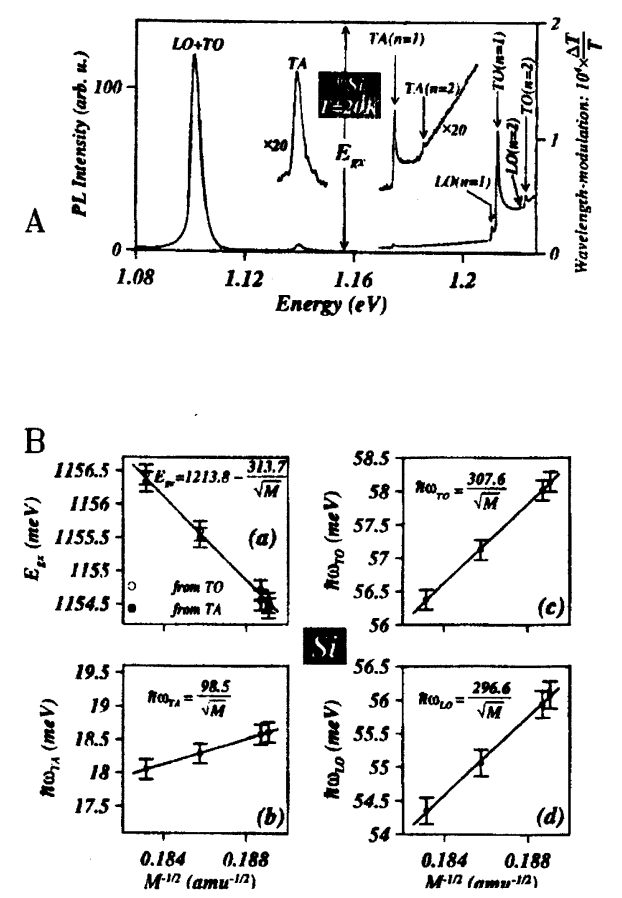

Figure 18. A - Photoluminescence (PL) and wavelength - modulated transmission (WMT) spectra of isotopically enriched ${ }^{30}$ Si recorded at 20K ; B - The excitonic indirect band gap and the associated phonon energies as a function of $\mathrm{M}$ (after [109])

Considering the series of Ge, $\mathrm{Si}$, GaAs, $\mathrm{ZnSe}, \mathrm{CuBr}$, for example, the 3d states of the first constituent play an increasing role in determining the band structure. In Ge these states can be considered as localized core states (atomic energy level $\approx-30 \mathrm{eV}$ ). Already, however, in GaAs they have moved up in energy by $10 \mathrm{eV}$, and their hybridization with the top of the valence band affects the gap (see e.g. [1] and reference therein). Proceeding further in the series, this effect becomes more important, and in $\mathrm{CuBr}$ and $\mathrm{Cu} 3 \mathrm{~d}$ states even overlap in energy with halogen p-states, with which they strongly hybridize. Therefore, we cannot exclude that the main reason for the opposite sign of the isotopic effect in these compounds may be connected to the different character of the d-electron-phonon interactions in these semiconductors [22].

The change of the indirect gap of diamond between pure ${ }^{12} \mathrm{C}$ and ${ }^{13} \mathrm{C}$ has been determined by Collins et al. [86], using for this purpose the luminescence spectra of diamond. The luminescence spectra of the natural $\left({ }^{12} \mathrm{C}\right)$ and synthetic $\left({ }^{13} \mathrm{C}\right)$ diamond were investigated by Collins et al. [86], Ruf et al [118]. Fig. 19 compares the edge luminescence for a natural diamond with that for a synthetic diamond. The peaks labelled A, B and C are due, respectively, to the recombination of a free exciton with the emission of transvers-acoustic, transverse-optic and longitudinal-optic phonons having wavevector $\pm \mathrm{k}_{\min }$ and quanta (in ${ }^{12} \mathrm{C}$ diamond) $[116,117]$

$\hbar \omega_{T A}=87 \pm 2, \hbar \omega_{T O}=141 \pm 2, \hbar \omega_{L O}=163 \pm 1 \mathrm{meV}$

Features $\mathrm{B}_{2}$ and $\mathrm{B}_{3}$ are further free-exciton processes involving the above TO phonon with one and two zonecentre optic phonons, respectively.

Boron forms an effective-mass-like acceptor in diamond, and both specimens used in Fig. 19 are slightly semiconducting with uncompensated boron concentrations around $5 \cdot 10^{16} \mathrm{~cm}^{-3}$ in the natural diamond and 3 $\cdot 10^{16} \mathrm{~cm}^{-3}$ in the synthetic diamond. Peaks labelled D are associated with the decay of excitons bound to the 


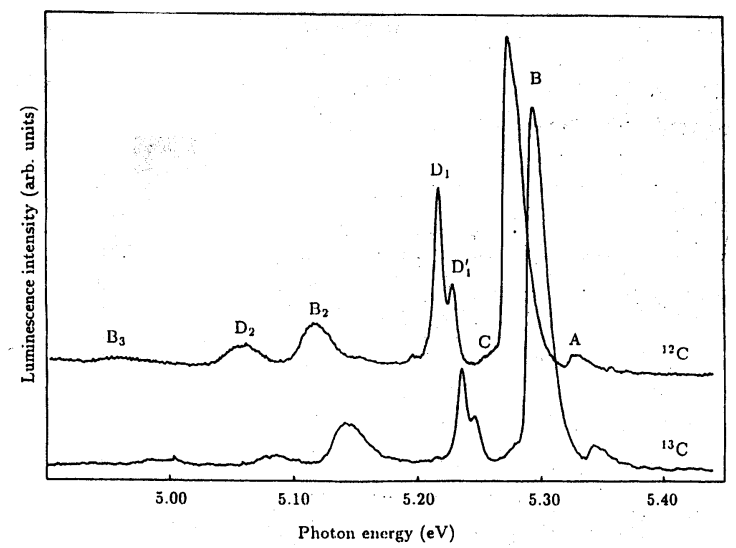

Figure 19. Spectra measured at $77 \mathrm{~K}$ of the phonon - assisted free exciton cathodoluminescence feature (A, B and C) and the phonon-assisted bound - exciton features (D) from a natural semiconducting ${ }^{12} \mathrm{C}$ diamond and a ${ }^{13} \mathrm{C}$ synthetic diamond (After [86])

boron acceptors (for details see [86]). Comparison of the data from the two diamonds shows that the zero-phonon lines $\mathrm{D}_{0}$ and $\mathrm{D}_{0}^{\imath}$ are $14 \pm 0.7 \mathrm{meV}$ higher for ${ }^{13} \mathrm{C}$ than for ${ }^{12} \mathrm{C}$ diamond, and that the LO and TO phonon energies are lower by a factor of 0.96 , equal within experimental error to the factor $(12 / 13)^{1 / 2}$ expected to first order when the lattice is changed from ${ }^{12} \mathrm{C}$ to ${ }^{13} \mathrm{C}$. The low-energy thresholds of the free-exciton peaks $\mathrm{A}, \mathrm{B}$ and $\mathrm{C}$ are given by $[116]$ :

$$
\mathrm{E}_{t h}(\mathrm{~A})=\mathrm{E}_{g x}-\hbar \omega_{T A} ; \mathrm{E}_{t h}(B)=\mathrm{E}_{g x}-\hbar \omega_{T O} \text { and } \mathrm{E}_{t h}(\mathrm{C})=\mathrm{E}_{g x}-\hbar \omega_{L O}
$$

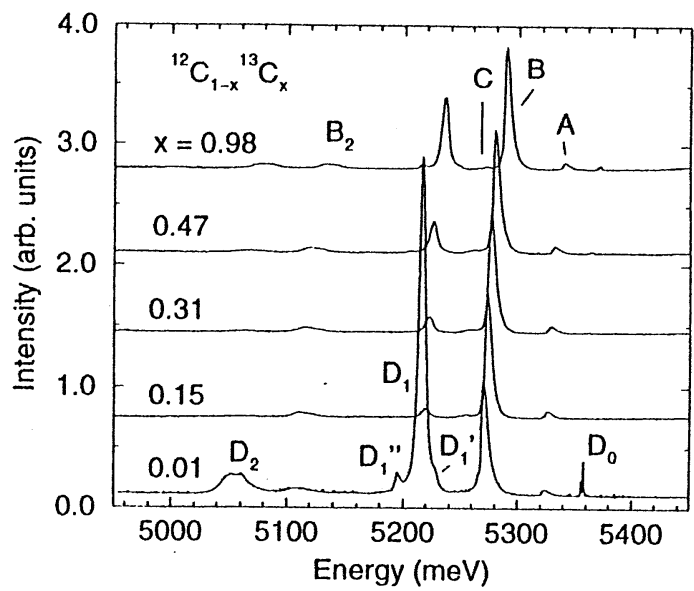

Figure 20. Cathode-luminescence spectra of isotopically modified diamond at 36 K. Intrinsic phonon-assisted recombination peaks are labelled in the top spectrum, those from boron-bound excitons in that at the bottom. The spectra are normalized to the intensity of the B peak and vertically offset for clarity (After [118])

As was shown by Collins et al. the predicted thresholds are entirely consistent with the experimental data. From the results of Collins et al. it was concluded that the dominant contribution arises from electron-phonon coupling, and that there is a smaller contribution due to a change in volume of the unit cell produced by changing the isotope. These two terms were calculated as $13.5 \pm 2.0$ and $3.0 \pm 1.3 \mathrm{meV}$ respectively. The more detailed and quantitative investigations of $\mathrm{E}_{g} \sim \mathrm{f}(x)$, where $\mathrm{x}$ is the isotope concentration, were done by Ruf et al. [118], where was studied five samples of diamond with different concentrations x (Fig. 20). From these data Ruf et al determined the linear variation of $\mathrm{E}_{g} \sim \mathrm{f}(x)$ for diamond (Fig. 21). Linear fits the experimental data of Ruf et al. , (solid line in Fig. 21) yield a slope of $14.6 \pm 0.5 \mathrm{meV} / \mathrm{amu}$, close to the theoretical predictions.

\subsection{Insulators - giant isotope effect}

\subsubsection{Renormalization of energy of band-to-band transitions}

Isotopic substitution only affects the wavefunction of phonons; therefore, the energy values of electron levels in the Schrődinger equation ought to have remained the same. This, however, is not so, since isotopic substitution modifies not only the phonon spectrum, but also the constant of electron-phonon interaction (see above). It is for this reason that the energy values of purely electron transition in molecules of hydride and deuteride are found to be different [119]. This effect is even more prominent when we are dealing with a solid [120]. Intercomparison of absorption spectra for thin films of $\mathrm{LiH}$ and $\mathrm{LiD}$ at room temperature revealed that the longwave maximum (as we 


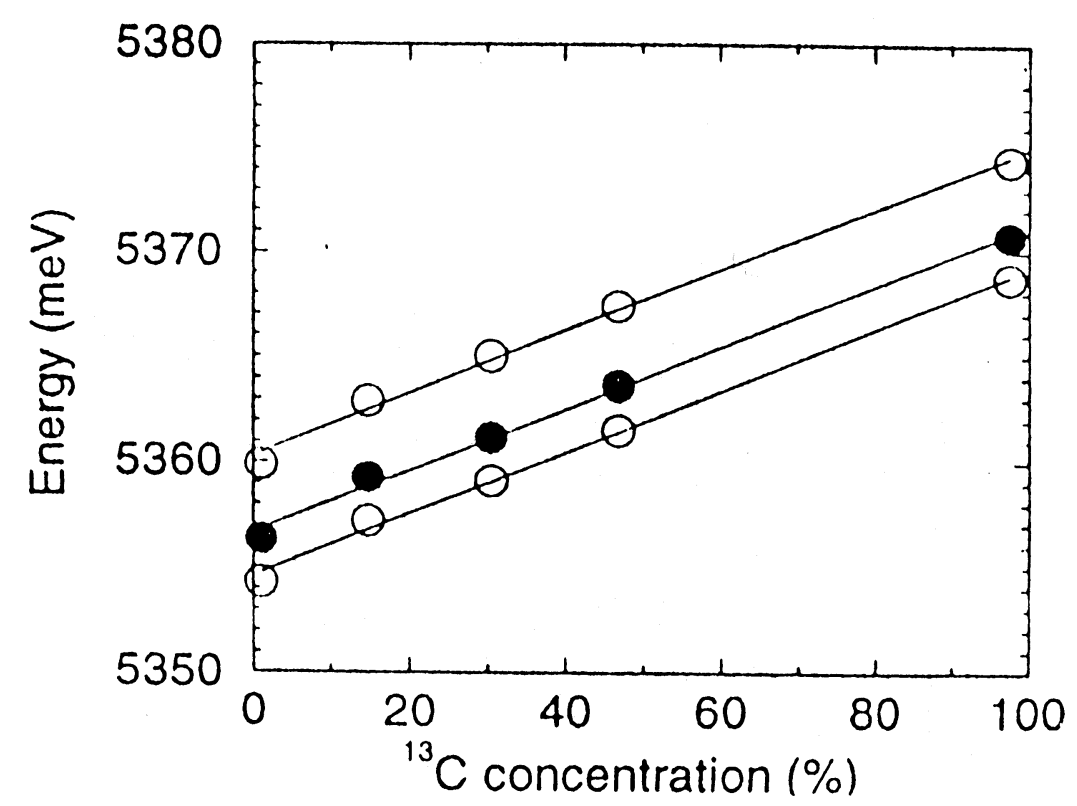

Figure 21. Energy of the $D_{0}$ multiplet in isotopic diamond at $36 \mathrm{~K}$. The filled symbols are for the main component, the open ones for weaker side peaks. The solid lines are linear fits to the data (After [118])

know now, the exciton peak [121]) moves $64.5 \mathrm{meV}$ towards the shorter wavelengths when H is replaced with D. For obvious reasons this fundamental result could not then receive consistent and comprehensive interpretation, which does not be little its importance even today. As will be shown below, this effect becomes even more pronounced at low temperatures (see, also [122]).

The mirror reflection spectra of mixed and pure LiD crystals cleaved in liquid helium are presented in Fig. 22. For comparison, on the same diagram we have also plotted the reflection spectrum of LiH crystals with clean surface. All spectra have been measured with the same apparatus under the same conditions. As the deuterium concentration increases, the long-wave maximum broadens and shifts towards the shorter wavelengths. As can clearly be seen in Fig. 22, all spectra exhibit a similar long-wave structure. This circumstance allows us to attribute this structure to the excitation of the ground (Is) and the first excited (2s) exciton states. The energy values of exciton maxima for pure and mixed crystals at $2 \mathrm{~K}$ are presented in Table 4 . The binding energies of excitons $\mathrm{E}_{b}$, calculated by the hydrogen-like formula, and the energies of interband transitions $\mathrm{E}_{g}$ are also given in Table 4 .

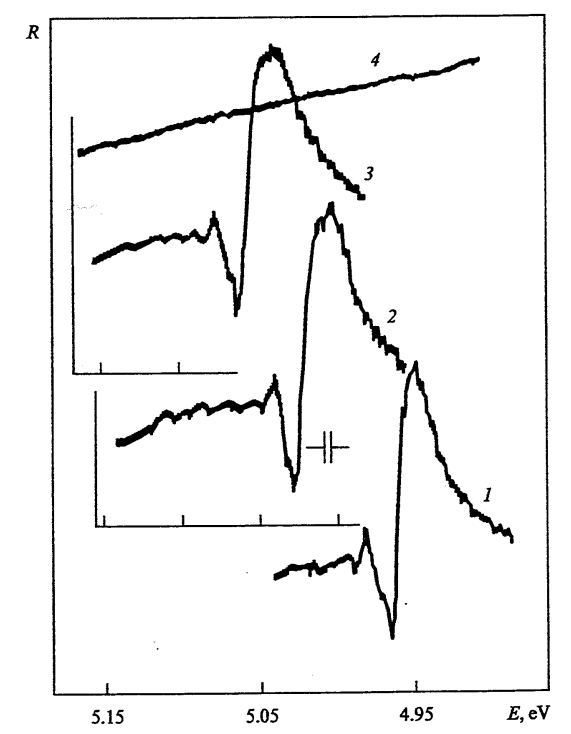

Figure 22. Mirror reflection spectra of crystals: $\mathrm{LiH}$, curve $1 ; \mathrm{LiH}_{x} \mathrm{D}_{1-x}$ curve 2 ; and $\mathrm{LiD}$, curve 3 at $4.2 \mathrm{~K}$. Light source without crystals, curve 4 (After [123])

The ionization energy, found from the temperature quenching of the peak of reflection spectrum of the $2 \mathrm{~s}$ state in $\mathrm{LiD}$ is $12 \mathrm{meV}$. This value agrees fairly well with the value of $\Delta E_{2 s}$ calculated by the hydrogen-like formula. Moreover, $\mathrm{E}_{b}=52 \mathrm{meV}$ for LiD agrees well with the energy of activation for thermal quenching of free-exciton 
luminescence in these crystals [124].

Going back to Fig. 22, it is hard to miss the growth of $\Delta_{12}$, [123], which in the hydrogen-like model causes an increase of the exciton Rydberg with the replacement of isotopes (see below Fig. 24) [124]. When hydrogen is completely replaced with deuterium, the exciton Rydberg (in the Wannier-Mott model) increases by $20 \%$ from 40 to $50 \mathrm{meV}$, whereas $\mathrm{E}_{g}$ exhibits a $2 \%$ increase, and at $2 \div 4.2 \mathrm{~K}$ is $\Delta \mathrm{E}_{g}=103 \mathrm{meV}$. This quantity depends on the temperature, and at room temperature is $73 \mathrm{meV}$, which agrees well enough with $\Delta \mathrm{E}_{g}=64.5 \mathrm{meV}$ as found in the paper of Kapustinsky et al. The continuous change of the exciton Rydberg was earlier observed in the crystals of solid solutions $\mathrm{A}_{3} \mathrm{~B}_{5}[36,125-127]$ and $\mathrm{A}_{2} \mathrm{~B}_{6}[128,129]$. Isotopic substitution of the light isotope $\left({ }^{32} \mathrm{~S}\right)$ by the heavy one $\left({ }^{34} \mathrm{~S}\right)$ in $\mathrm{CdS}$ crystals [96]) reduces the exciton Rydberg, which was attributed to the tentative contribution from the adjacent electron bands (see also Bobrysheva et al. [62]), which, however, are not present in $\mathrm{LiH}$ (for more details see $[130,131]$ ). The single-mode nature of exciton reflection spectra of mixed crystals $\mathrm{LiH}_{x} \mathrm{D}_{1-x}$ agrees qualitatively with the results obtained with the virtual crystal model (see e.g. [132, 133]), being at the same time its extreme realization, since the difference between ionization potentials $(\Delta \zeta)$ for this compound is zero. According to the virtual crystal model, $\Delta \zeta=0$ implies that $\Delta \mathrm{E}_{g}=0$, which is in contradiction with the experimental results for $\mathrm{LiH}_{\mathrm{x}} \mathrm{D}_{1-\mathrm{x}}$ crystals. As was shown above the change in $\mathrm{E}_{g}$ caused by isotopic substitution has been observed for many broad-gap and narrow-gap semiconductor compounds.

Table 3. The change of the value of the exciton binding energy $\left(\Delta \mathrm{E}_{B}\right)$, band-to-band transitions $\left(\Delta E_{g}\right)$ at the $100 \%$ isotopical substitution. All values indicate in $\mathrm{meV}_{\text {or }} \mathrm{cm}^{-1}$. (After Plekhanov, 1998)

$\begin{array}{lll}\text { Compound } & \Delta E_{g} & \Delta E_{B} \\ \mathrm{Ge}^{70} \rightarrow G e^{76} & \text { indirect (L-point) } 2.1 \mathrm{mev}=16.94 \mathrm{~cm}^{-1}[1,2,3] & - \\ \mathrm{GaAs}\left(\mathrm{Ga}^{69} \rightarrow G a^{71}\right) & \text { direct ( } \Gamma \text { point }) 0.85 \mathrm{mev}=10.5 \mathrm{~cm}^{-1}[4] & - \\ \mathrm{Cu}_{2} \mathrm{O}\left(\mathrm{O}^{16} \rightarrow \mathrm{O}^{18}\right) & \text { direct (yel) } 2.2 \mathrm{mev}=18 \mathrm{~cm}^{-1}[5] & - \\ \mathrm{Cu}_{2} \mathrm{O}\left(\mathrm{O}^{16} \rightarrow O^{18}\right) & \text { direct (green) } 2.2 \mathrm{mev}=18 \mathrm{~cm}^{-1}[5] & - \\ \mathrm{CdS}\left(\mathrm{S}^{32} \rightarrow \mathrm{S}^{34}\right) & - & -1.6 \mathrm{meV}[7] \\ \mathrm{CdS}\left(\mathrm{Cd}^{112} \rightarrow C d^{n a t}\right) & \text { direct } 0.003 \mathrm{meV} & 0.4 \mathrm{meV}[8] \\ \mathrm{CuCl}\left(\mathrm{Cu}^{63} \rightarrow \mathrm{Cu}^{65}\right) & \left(\Gamma-\text { point }-0.155 \mathrm{meV}=-1.24 \mathrm{~cm}^{-1}[9]\right. & - \\ \mathrm{ZnO}\left(\mathrm{O}^{16} \rightarrow \mathrm{O}^{18}\right) & \left(\Gamma-\text { point) } 6.82 \mathrm{meV}=55 \mathrm{~cm}^{-1}[10,11]\right. & - \\ \mathrm{ZnO}\left(\mathrm{Zn}^{64} \rightarrow Z n^{68}\right) & (\Gamma-\text { point }) 1.5 \mathrm{meV}=12 \mathrm{~cm}^{-1}[10,11] & - \\ \mathrm{C}^{12} \rightarrow C^{13} & \text { indirect }(\Gamma-\mathrm{point}) 13.6 \mathrm{meV}^{13}=109.7 \mathrm{~cm}^{-1}[12] & - \\ \mathrm{LiH}(\mathrm{H} \rightarrow D) & \text { direct } 103 \mathrm{meV}=831 \mathrm{~cm}^{-1}[15,16,17] & 10 \mathrm{meV}[16] \\ \mathrm{LiH}\left(\mathrm{Li}^{6} \rightarrow L i^{7}\right) & \text { direct } 5 \mathrm{meV}=41 \mathrm{~cm}^{-1}[16] & 3 \mathrm{meV}[16]\end{array}$

\section{REFERENCES}

1. V.F. Agekyan et al, Fizika Tverdogo Tela, 31, 101 (1989) (in Russian) .

2. C. Parks et al, Phys. Rev., B49, 14244 (1994).

3. J.J.Haller, J. Appl. Phys. 77, 2857 (1995).

4. N. Garro et al, Phys. Rev., B54, 4732 (1996).

5. F.I. Kreingol'd et al, Pis'ma v ZETPH, 23, 679 (1976) (in Russian).

6. F.I. Kreingol'd, Fizika Tverdogo Tela, 27, 2839 (1985) (in Russian).

7. F.I. Kreingol'd et al, ibid, 26, 3490 (1984) (in Russian).

8. J.M. Zhang et al, Phys. Rev. B57, 9716 (1998)

9. N. Garro et al, Solid State Commun., 98, 27 (1996).

10. F.I. Kreingol'd, Fizika Tverdogo Tela 20, 3138 (1978) (in Russian).

11. F.I. Kreingol'd and B.S. Kulinkin, ibid, 28, 3164 (1986) (in Russian).

12. T. Collins et al, Phys. Rev. Lett., 65, 891 (1990).

13. R.M. Chrenko, J. Appl. Phys. 63, 5873 (1988).

14. K.S. Hass et al, Phys. Rev. B54, 7171 (1992).

15. A.A. Klochikhin and V.G.Plekhanov, Fizika Tverdogo Tela, 22,585 (1980) (in Russian).

16. V.G. Plekhanov, Uspekhi Fiz. Nauk, 167, 577 (1997); Plekhanov V.G and Plekhanov N.V. Phys. Lett. A313, 231(2003).

17.A.F. Kapustinsky et al, Physicochimica USSR, 7, 799 (1937).

All of these results are documented in Table 3, where the variation of $\mathrm{E}_{g}, \mathrm{E}_{b}$, are shown at the isotope effect. We should highlighted here that the most prominent isotope effect is observed in LiH crystals, where the dependence of $\mathrm{E}_{b}=\mathrm{f}\left(\mathrm{C}_{H}\right)$ is also observed and investigated. To end this section, let us note that $\mathrm{E}_{g}$ decreases by $97 \mathrm{~cm}^{-1}$ when ${ }^{7} \mathrm{Li}$ is replaced with ${ }^{6} \mathrm{Li}$ (see Table 3 ).

\subsubsection{Renormalization of binding energy of Wannier - Mott excitons by isotopic effect}

It was in the original work [121] where the exciton binding energy $\mathrm{E}_{b}$ was found to depend on the isotopic composition that this change in $\mathrm{E}_{b}$ was attributed to the exciton-phonon interaction (originally with LO phonons). The preferential interaction of excitons with LO phonons in LiH (LID) crystals was later repeatedly demonstrated in the luminescence spectra [134] and resonant Raman light scattering [135], which consist of a phononless line (in the former case) and its LO repetitions. The effects of the Frohlich mechanism of exciton-phonon interaction on the energy spectrum of Wannier-Mott exciton has been considered over and over again $[17,63,136]$. Today we 
know that the main consequences of electron and hole interaction in excitons with polarization vibrations are the static screening of the lattice charges (introducing $\varepsilon_{0}$ ) and the change in the effective masses of the particles. Both these effects of electron-(hole)- phonon interaction can easily be taken into account, and lead to a change in the exciton Rydberg $\mathrm{E}_{b}$. These corrections do not destroy the hydrogen-like structure of the exciton spectrum. At the same time, the non-coulombic corrections to the electron-hole Hamiltonian modify the hydrogen-like structure removing, for example, degeneration of levels with respect to orbital and magnetic quantum numbers (see e.g. [1] and references therein). The very fact, however, that the problem of renormalization of energy spectra of Wannier - Mott excitons does not admit an exact solution even in the limiting cases, often gives rise to a situation in which there is no agreement between the results obtained by different authors. Starting with the classical works of Haken [63], all papers may be divided into two broad classes depending on how they take deal with the Coulomb interaction: between 'bare' electrons and holes, or between electrons and holes in the polaron state. In other words, first the interaction of band electrons and holes with LO optical phonons is taken into account, and then of the Coulomb interaction between electrons and holes clad in the 'polarization coats' is considered. As will be shown below, the study of exciton-phonon interaction in crystals with isotopic effect not only provides entirely new information, but also allows us to reconstruct experimentally the values of Frohlich and Coulomb interaction constants. From Fig. 23 we see that when hydrogen is completely replaced with deuterium, the binding energy of the exciton exhibits a $20 \%$ increase from 42 to $52 \mathrm{meV}$ [121]. It is easy to see that in the model of virtual crystal the binding energy of the exciton in LiT crystals [137] must be equal to $57 \mathrm{meV}$ (see Fig. 23). Hence it follows that in the linear approximation the isotopic dependence of binding energy of Wannier - Mott excitons may be expressed as

$$
\mathrm{E}_{b}=\mathrm{E}_{b}(0)(1+\gamma)
$$

where $\mathrm{E}_{b}(0)$ is the purely coulombic binding energy of the exciton (in the frozen lattice), which in our case is equal to $31.5 \mathrm{meV}$, and the angular coefficient is $\beta=12.18 \mathrm{meV} / \mathrm{M}$, where $\mathrm{M}$ is the reduced mass of ions of lithium and hydrogen (deuterium, tritium) ions; $\gamma=\beta \mathrm{M} / \mathrm{E}_{b}(0)$ [127]. From the standard equation for the Coulomb binding energy of the exciton

$$
\begin{aligned}
& \mathrm{E}_{b}=\frac{e^{4} \mu}{\hbar^{2} \varepsilon_{\infty}^{2}} \quad\left(21^{a}\right) \\
& \text { we get the dimensionless constant of Coulomb interaction: } \\
& \eta^{2}=\frac{E_{b}(0)}{\hbar \omega_{L O}}=0.47 . \quad(51)
\end{aligned}
$$

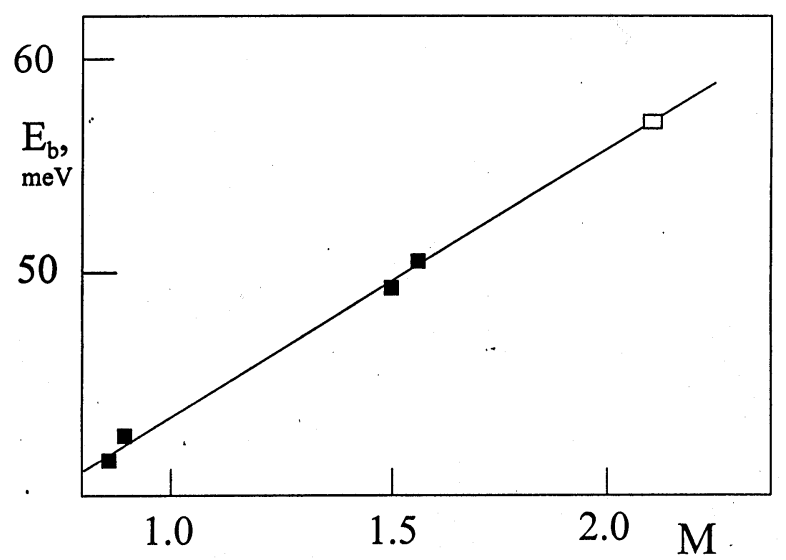

Figure 23. Binding energy of Wannier - Mott excitons as function of reduced mass of ions based on values of reduced mass of ions for ${ }^{6} \mathrm{LiH},{ }^{7} \mathrm{LiH},{ }^{6} \mathrm{LiD},{ }^{7} \mathrm{LiD}$ and $\mathrm{LiT}$ (after [123])

Comparing the value of $\eta^{2}=0.47$ and the constant of Fröhlich exciton-phonon interaction $\mathrm{g}^{2}=0.33$ [22]) we see that they are close enough. This implies that both the Fröhlich and the Coulomb interactions between electrons (holes) and LO phonons in exciton must be treated with equal attention, as has already been emphasized in Klochikhin's paper [77]. This paper deals from the start with 'bare' electrons and holes, and all renormalizations are calculated in the two-particle configuration. Such an approach enables us to avoid the considerable difficulty which arises when polarons [139] are used as start-up particles. This difficulty is primarily associated with the fact that the momentum of each particle is conserved when the particles are treated separately, whereas it is the center-of-mass momentum that is conserved when a pair moves as a whole. As demonstrated in Klochikhin [77], this approach also makes it possible to calculate the higher-order corrections to the exciton-phonon interaction. It was also shown that the use of the pole parts of polaron Green functions in place of complete expressions in Sak [139] and Mahanti and Varma [140] leads to a situation when the corrections of the order of $\eta^{2} g^{2}$ and $g^{4}$ to the potential energy are lost because the corrections to the vertex parts and Green functions cancel out. The quantity lost is of the same order $\left(\mathrm{g}^{2}\right)$ as the correction to the residue but has the opposite sign [139, 140]. The approach developed in Klochikhin [77] allowed the calculation of corrections of the order $\eta^{2} g^{2}$ and $\mathrm{g}^{4}$, the latter is comprised of the correction to the Frohlich vertex and the correction to the Green functions in the exciton-phonon loop. It is 
important that the latter have opposite sign and cancel out exactly in the limit $\mathrm{E}_{b} \ll \hbar \omega_{L O}$. As a result, because of the potential nature of the start-up Coulomb interaction, the correction to the Coulomb vertex of the order $\eta^{2} g^{2}$ does not vanish. As a result, the following expression was obtained in Klochikhin's paper for the binding energy $\mathrm{E}_{b}$ of Wannier-Mott exciton when $\mathrm{E}_{b} \ll \hbar \omega_{L O}$ (the spectrum of exciton remains hydrogen-like):

$$
\mathrm{E}_{b}=\hbar \omega_{L O}\left[\frac{\eta^{2}-\mathrm{g}^{2}+\eta^{2} \mathrm{~g}^{2}(\mathrm{c}+\mathrm{v})}{2}\right]^{2}
$$

where $\mathrm{c}, \mathrm{v}=\left(\mathrm{m}_{c, v}, / \mu\right)^{1 / 2}$, and $\mathrm{m}_{c}, \mathrm{~m}_{v}$ are the electron and hole masses. Now $\mathrm{E}_{b}$ depends explicitly on $\mathrm{g}^{2}$ (the Fröhlich constant of exciton-phonon interaction), and hence depends on the isotopic composition of the lattice, whereas the standard expression for the binding energy $\mathrm{E}_{b}=\hbar \omega_{L o}\left(\eta^{2}-\mathrm{g}^{2}\right)=\mathrm{e}^{4} \mu / \varepsilon_{0}^{2} \hbar^{2}$, which describes the exciton spectrum of many semiconductors accurately enough, exhibits no dependence on the isotopic effect. In the case of Eq. (52) the exciton spectrum remains hydrogen-like. When the higher-order corrections are taken into account, Eq. (52) becomes

$$
\mathrm{E}_{b}=\frac{e^{4} \mu}{2 \varepsilon_{0} \hbar^{2}}\left[1+\mathrm{g}^{2} \varepsilon_{0} \frac{m_{c}+\mathrm{m}_{v}}{\varepsilon_{\infty}}+\mathrm{g}^{4} \frac{\varepsilon_{0}}{\varepsilon_{\infty}}\left(\zeta_{1}+\zeta_{2} \frac{1-\varepsilon_{\infty}}{\varepsilon_{0}}\right)\left(m_{c}+\mathrm{m}_{v}\right)\right]
$$

The order-of-magnitude evaluation of the coefficients $\zeta_{1}, \zeta_{2}$ gives $\zeta_{1} \approx 0.15$ and $\zeta_{2} \approx 0.02 ;$ when $\mathrm{g}^{2}\left(\mathrm{~m}_{c}+\mathrm{m}_{v}\right)$ $\ll 3.3$, the correction of the order $\eta^{2} \mathrm{~g}^{4}$ is much less than the term of the order $\eta^{2} \mathrm{~g}^{2}[77]$. Setting $\mathrm{m}_{v} / \mathrm{m}_{c}=3.5$ and $\mathrm{g}^{2} / \eta^{2}=\mathrm{I}-\varepsilon_{0} / \varepsilon_{\infty}$, and $\left.\left(\varepsilon_{\infty} / \varepsilon_{0}\right)=\left(\omega_{T O} / \omega_{L O}\right)=1 / 3.5\right)$. In Ref. [122] it was found that $\mathrm{E}_{b}($ theor $)=48$ and $42 \mathrm{meV}$ for $\mathrm{LiD}$ and $\mathrm{LiH}$ respectively. Comparing these results with the experimental values (see Table 4) we observe good agreement between theory and experiment. Hence follows a natural conclusion that the isotopic dependence of the exciton binding energy is due primarily to the Frohlich interaction mechanism between excitons and phonons.

Table 4. Values of the energy of maxima (in $\mathrm{meV}$ ) in exciton reflection spectra of pure and mixed crystals at $2 \mathrm{~K}$, and energies of exciton binding $\mathrm{E}_{\mathrm{b}}$, band-to-band transitions $\mathrm{E}_{\mathrm{g}}$ (After [1]).

\begin{tabular}{|c|c|c|c|c|c|}
\hline Energy, meV & $\mathrm{LiH}$ & $\mathrm{LiH}_{0.82} \mathrm{D}_{0.18}$ & $\mathrm{LiH}_{0.40} \mathrm{D}_{0.60}$ & $\mathrm{LiD}$ & ${ }^{6} \mathrm{LiH}(78 \mathrm{~K})$ \\
\hline $\mathrm{E}_{1 \mathrm{~s}}$ & 4950 & 4967 & 5003 & 5043 & 4939 \\
\hline $\mathrm{E}_{2 \mathrm{~s}}$ & 4982 & 5001 & 5039 & 5082 & 4970 \\
\hline $\mathrm{E}_{\mathrm{b}}$ & 42 & 45 & 48 & 52 & 41 \\
\hline $\mathrm{E}_{\mathrm{g}}$ & 4992 & 5012 & 5051 & 5095 & 4980 \\
\hline
\end{tabular}

In the preceding section was shown that isotopic substitution affects not only $\mathrm{E}_{b}$, but also $\mathrm{E}_{g}$. For the LiH-LiD system at low temperatures the difference is $\Delta \mathrm{E}_{g}=103 \mathrm{meV}$. Apart from the zero oscillations considered in the previous section $[97,141]$, this change is also contributed to by the polaron shift which explicitly depends on the temperature (for details see [142]).

\subsubsection{Nonlinear dependence of exciton binding and bandgap energies on isotope concentration}

A principal matter for further theoretical development is the question concerning the effect of crystal lattice disordering on the binding energy $\mathrm{E}_{B}$ of Wannier-Mott exciton. This problem has been treated theoretically in the papers of Elliott and coworkers $[143,144]$, where they study the effect of weak disordering on $\mathrm{E}_{B}$ (the disordering energy is comparable with $\left.\mathrm{E}_{B}\right)$. The binding energy indicated in the papers was calculated under the coherent potential approximation by solving the Bethe-Salpeter equation as applied to the problem of Wannier-Mott exciton in disordered medium. One of the principal results of this paper [143] is the nonlinear dependence of $\mathrm{E}_{B}$ on the concentration. As a consequence, the binding energy $\mathrm{E}_{B}$ at half-and-half concentrations is less than the value derived from the virtual crystal model. The exciton binding energy is reduced because the energy $\mathrm{E}_{g}$ is less owing to the fluctuation smearing of the edges of the conduction and valence band. This conclusion is in qualitative agreement (although not in quantitative agreement, the discrepancy being about an order of magnitude [143]) with the experimental results for the mixed crystal $\mathrm{GaAs}_{1-x} \mathrm{P}_{x}$ with $\mathrm{x}=0.37$, where the reflection spectra exhibited two exciton maxima (see also Fig. 12) used for finding the value of $\mathrm{E}_{b}$ ([124] and references therein). Let us add that the pivotal feature of the model of Elliott and coworkers is the short-range nature of the Coulomb potential (for more details see [123]).

Before the comparison of our experimental results with the theory developed by Elliott and Kanehisa, it would be prudent to briefly review main properties of their theoretical model. According to Ref. 143 this model considers an exciton with a direct gap of a semiconductor alloy. Such a system consists of an electron (particle 1) in the conduction band (c) with mass $\mathrm{m}_{c}$ and a hole (particle 2) in the valence band ( $\mathrm{v}$ ) with mass $\mathrm{m}_{v}$. The problem of the exciton in disordered systems is to solve the Hamiltonian

$\mathrm{H}=\vec{p}^{2} / 2 \mathrm{~m}_{c}+\vec{p}^{2} / 2 \mathrm{~m}_{v}+\mathrm{u}\left(\vec{r}_{1}-\vec{r}_{2}\right)+\mathrm{V}_{c}\left(\vec{r}_{1}\right)+\mathrm{V}_{v}\left(\vec{r}_{2}\right)$

with both the Coulomb interaction $\mathrm{u}$ and the potential $\mathrm{V}_{v}$ due to disorder $(\nu=\mathrm{c}, \mathrm{v})$. Reference 143 neglected disorder - induced interband mixing. As it is well known, in place of the electron-hole coordinates, $\left(\vec{r}_{1}, \vec{p}_{1}\right)$ and $\left(\vec{r}_{2}, \vec{p}_{2}\right)$, one may introduce the center-of-mass and relative coordinates, $(\vec{R}, \vec{P})$ and $(\vec{r}, \vec{p})$ to rewrite $(54)$ as

$\mathrm{H}=\vec{p}^{2} / 2 \mathrm{~m}_{r}+\mathrm{u}(\vec{r})+\vec{P}^{2} / 2 \mathrm{M}+\mathrm{V}_{c}\left(\vec{R}+\mathrm{m}_{v} \vec{r} / \mathrm{M}\right)+\mathrm{V}_{v}\left(\vec{R}-\mathrm{m}_{c} \vec{r} / \mathrm{M}\right)$

where $\mathrm{m}_{r}$ and $\mathrm{M}$ are the reduced and total masses, respectively. Because of the random potential, the translational and relative degrees of freedom cannot be decoupled. This is essentially difficult when considering the two-body problem in a disordered system [140]. However, when the exciton state in question is well separated from other states so that the energy spacing is much larger than the translational width and disorder, one can forget about the relative motion and just apply any single-particle alloy theory (see, e.g. Ref. [145] and references 
therein) solely to their translational motion. For each exciton state the translational part of Hamiltonian in this case is

$$
\mathrm{H}_{t}=\vec{P}^{2} / 2 \mathrm{M}+\overline{\mathrm{V}}_{c}(\vec{R})+\overline{\mathrm{V}}_{v}(\overline{\mathrm{R}}) \quad(56)
$$

Here $\overline{\mathrm{V}}_{c}$ and $\overline{\mathrm{V}}_{v}$ are averages of $\mathrm{V}_{c}$ and $\mathrm{V}_{v}$. This approach is very similar to the Born-Oppenheimer adiabatic approximation. Such situations hold in some mixed alkali halide crystals and probably $\mathrm{A}_{2} \mathrm{~B}_{6}$ crystals. On the contrary, when the exciton binding energy is comparable to the disorder energy, the adiabatic approximation breaks down, and it is essential to take into account the effect of disorder on both the translational and relative motions. This is the case with the Wannier-Mott exciton in $\mathrm{A}_{3} \mathrm{~B}_{5}$ alloys, for which the Elliott and Kanehisa model was developed. In this case the solution task is to start from the independent electron and hole by neglecting $\mathrm{u}$ in (56) and then to take into consideration the Coulomb interaction between the average electron and average hole. A further simplified approach adopted in the literature [145] in solving the Bethe-Salpiter [146] equation is to suppose a free-electron-like one particle Green's function with a built-in width to allow for the random potential due to disorder. In the cited theoretical model, the average (or "virtual crystal") gap is given by

$\mathrm{E}_{g}^{v c}(\mathrm{x})=\mathrm{E}_{0}+\left(\delta_{c}-\delta_{v}\right)(\mathrm{x}-1 / 2)$

where $\mathrm{E}_{0}$ is average gap, $\delta_{c}, \delta_{v}$ are the values of the fluctuation broadening of the conduction and valence bands, respectively. Reference 100 also assumed the Hubbard density of states for both the conduction and valence bands with width $\mathrm{W}_{c}$ and $\mathrm{W}_{v}$, respectively, as well as similar dispersion in both bands. With this assumption the exciton binding energy has been calculated according to the CPA model (see, also [132]). It should be added here the ley feature of the model developed in Ref. 143 is the short-range nature of the Coulomb potential.

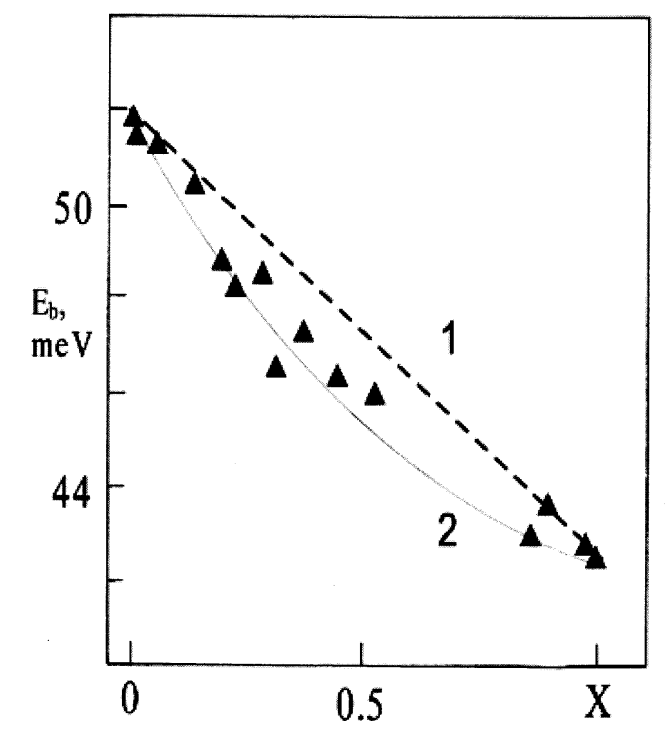

Figure 24. Concentration dependence of binding energy of Wannier-Mott exciton:1 - approximation of virtual crystal model; 2 calculation according to Eq. (1.62); experimental points indicated by triangles (After [123])

The data from Table 1 and other published sources (see [1, 22]) were used for plotting the energy $\mathrm{E}_{B}$ as a function of isotopic concentration $\mathrm{x}$ in Fig. 24. The values of binding energy $\mathrm{E}_{B}$ were calculated using the hydrogen-like formula (see below) with the energies of exciton levels of Is and 2 s states being found from the reflection spectra (see Fig. 22). Theoretical description of the binding energy of Wannier- Mott excitons as a function of $\mathrm{x}$ was based on the polynomial derived by Elliott and cowokers [143]:

$$
\begin{aligned}
& \mathrm{E}_{b}=\mathrm{E}_{b}^{c r y s}-\mathrm{E}_{\text {bow }}\left[\frac{1-W}{2 U_{0}}\right]-E_{\text {eff }}, \\
& \mathrm{E}_{e f f}=\mathrm{x}(1-x) \frac{\delta_{c} \delta_{v}}{W} \\
& \mathrm{E}_{b}^{c r y s}=\mathrm{U}_{0}+\frac{W}{2 U_{0}}-\mathrm{W} .
\end{aligned}
$$

where $\mathrm{W}=\mathrm{W}_{c}+\mathrm{W}_{v}$, and $\mathrm{W}_{c}$ and $\mathrm{W}_{v}$ are the widths of the conduction band and the valence band which are equal to $21 \mathrm{eV}$ [147] and $6 \mathrm{eV}$ [148] respectively. Here $\mathrm{E}_{b o w}$ is the curvature parameter found from the function $\mathrm{E}_{g} \propto f(\mathrm{x}) ; \delta_{c}$ and $\delta_{v}$ are the magnitudes of the fluctuation smearing of the valence band and the conduction band edges , $\delta_{c}=0.103 \mathrm{eV}$ and $\delta_{v}=-0.331 \mathrm{eV}$. As follows from Fig. 24, these values of the parameters give a good enough description of the nonlinear dependence of the binding energy of Wannier-Mott exciton in disordered medium. This agreement between theory and experiment once again proves the inherent consistency of the model proposed by Kanehisa and Elliott, since the isotopic substitution affects the short-range part of the interaction potential.

In this way, the nonlinear dependence of the binding energy of Wannier-Mott exciton is caused by isotopic disordering of the crystal lattice. As is see from Fig. 24 the exciton binding energy decreasing (relative linear law (VCA) - see dashed line in Fig. 24) in the vicinity of the middle meaning concentration really calls out the fluctuative broadening of the edge of the conduction and valence bands. In accordance with the theoretical model the last reason gives rise to the reduced $\mathrm{E}_{g}$ and there by the shallowing of the exciton levels and, respectively, the 
reduction of $\mathrm{E}_{b}$ (more details see $[1,22]$ ).

Below we report the first results of the quantitative study the dependence of the band-gap energy on the isotope effect for different compounds [149]. As was mentioned above isotopic substitution only affects on the wavefunctions of phonons; therefore, the energy values of electron levels in the Schrödinger equation ought to have remained the same. This, however, is not so, since isotopic substitution modifies not only the phonon spectrum, but also the constant of electron-phonon interaction. It is for this reason the energy values of purely electron transitions in molecules of hydride and deuteride are found to be different (see, e.g. [1]).

The main-square vibrational amplitude $\left\langle\mathrm{u}^{2}\right\rangle$ of an atom depends on the phonon frequencies and the eigenvectors, the atomic masses (at low temperatures), as well as the temperature (at high temperature). Isotope substitution results in a slightly different vibrational amplitudes (especially at low temperature) and phonon frequencies and in first approximation equivalent to changing the temperature (see also [150]). The mass dependence of $\left\langle\mathrm{u}^{2}\right\rangle$ becomes vanishingly small at temperature on the order or higher than the Debye temperature. Changes in either the isotope masses or temperature thus lead to changes in the band gap (see above Fig. 22) via the electron-phonon interaction, even at zero temperature in the case of mass changes. In general, the renormalization of the band gap [4] and its temperature dependence result from a complicated interplay of 1) first- and secondorder electron-phonon interactions that contribute to the energy of conduction and valence bands; 2) changes due to thermal or isotopic lattice expansion, and 3) changes in the phonon occupation numbers (see [150] and references therein).

The dependence of the band gap energy on isotopic composition has already been observed for insulators and lowest (indirect-direct) gap of different semiconductors (see also [1]). It has been shown to result primarily from the effect of the average isotopic mass on the electron-phonon interaction, with a smaller contribution from the change in lattice constant. This simplest approximation, in which crystals of mixed isotopic composition are treated as crystals of identical atoms having the average isotopic mass is referred to as virtual crystal approximation (VCA) [133]. Going beyond the VCA, in isotopically mixed crystals one would also expect local fluctuations in the band gap energy from statistical fluctuations in local isotopic composition within some effective volume, such as that of an exciton (see preceding section). As follows from Fig. 22. excitons in $\mathrm{LiH}_{x} \mathrm{D}_{1-x}$ crystals display a unimodal character, which facilitates the interpretation of their concentration dependence. Fig. 25 shows the concentration dependence of the energy of interband transition $\mathrm{E}_{g}$. As can be seen from Fig. 25 VCA method cannot describe observed experimental results. As will shown below this deviation from linear low (VCA approximation) is connected with isotope-induced-disorder in isotope mixed crystals $\mathrm{LiH}_{x} \mathrm{D}_{1-x}$.



Figure 25. Dependence of the interband transition energy $\mathrm{E}_{g}$ in mixed crystals $\mathrm{LiH}_{x} \mathrm{D}_{1-x}$ on the concentration $\mathrm{x}$. The straight dashed line is the linear dependence $\mathrm{E}_{g}=\mathrm{f}(\mathrm{x})$ in the virtual crystal model. The solid line corresponds to calculations using the polynom of second degree. Points derived from the reflection spectra indicated by crosses, and those from luminescence spectra by triangles (After $[1])$

The temperature and isotopic mas dependence of a given energy gap $\mathrm{E}_{g}\left(\mathrm{~T}, \mathrm{M}_{i}\right)$ can be described by average Bose-Einstein statistical factor $\mathrm{n}_{B}$ corresponding to an average phonon frequency $\theta_{i}$ as (see also [22, 92])

$\mathrm{E}_{g}\left(\mathrm{~T}, \mathrm{M}_{i}\right)=\mathrm{E}_{b a r}-\mathrm{a}_{r}\left(\frac{M_{n a t}}{M_{i}}\right)^{1 / 2}\left[1+2 \mathrm{n}_{\mathrm{B}}\right]$

where $\mathrm{n}_{B}=1 /\left(\exp \left(\frac{\theta_{i}}{\mathrm{~T}}\right)-1\right)$ and $\mathrm{E}_{b a r}$ and $\mathrm{a}_{r}$ the unrenormalized (bare) gap and the renormalization parameter, respectively. In the low-temperature limit, $\mathrm{T}<<\theta_{i}$, equation (62) reduces

$\mathrm{E}_{g}\left(\mathrm{~T}, \mathrm{M}_{i}\right)=\mathrm{E}_{\text {bar }}-\mathrm{a}_{r}\left(\frac{M_{n a t}}{M_{i}}\right)^{1 / 2}$

Here $\mathrm{E}_{g}\left(\mathrm{~T}, \mathrm{M}_{i}\right)$ is independent of temperature and proportional to $\left(1 / \mathrm{M}_{i}\right)^{1 / 2}$, whereas $\mathrm{a}_{r}$ is the energy difference between the unrenormalized gap $\left(\mathrm{M}_{i} \rightarrow \infty\right)$ and the renormalized value [150].

In the high-temperature limit, $\mathrm{T}>>\theta_{i}$ and Eq. (62) can be written as

$\mathrm{E}_{g}\left(\mathrm{~T}, \mathrm{M}_{i}\right)=\mathrm{E}_{b a r}-2 \mathrm{~T} \frac{a_{r}}{\theta}$, 
and $\mathrm{E}_{g}\left(\mathrm{~T}, \mathrm{M}_{i}\right)$ is independent of $\mathrm{M}_{i}$ [107]. The extrapolation of Eq. (64) to $\mathrm{T}=0 \mathrm{~K}$ can be used to determine the unrenormalized gap energy $\mathrm{E}_{b a r}$, i.e., the value that corresponds to atoms in fixed lattice position without vibrations (frozen lattice), from the measured temperature dependence of $\mathrm{E}_{g}(\mathrm{~T})$ in the high-temperature (i.e. linear in $\mathrm{T}$ ) region.

Using Eq. (63) it can be written the difference in energy $\Delta \mathrm{E}_{g}$ between a given energy gap in isotopically pure material $(\mathrm{LiH})$ and its isotope analogue $(\mathrm{LiD})$

$$
\Delta \mathrm{E}_{g}=\mathrm{E}_{g}\left(\mathrm{M}_{i}\right)-\mathrm{E}_{g}\left(\mathrm{M}_{n a t}\right)=\mathrm{a}_{r}\left[1-\left(\frac{M_{n a t}}{M_{i}}\right)^{1 / 2}\right],
$$

As can be seen from Table 3 and results of $\Delta \mathrm{E}_{g}$ at $2 \mathrm{~K}$ equals $\Delta \mathrm{E}_{g}=0.103 \mathrm{eV}$ and $\mathrm{E}_{g}(\mathrm{LiH}, \mathrm{T}=0 \mathrm{~K})=5.004$ $\mathrm{eV}$ (linear approximation and $\mathrm{E}_{g}(\mathrm{LiH}, \mathrm{T}=300 \mathrm{~K})=4.905 \mathrm{eV}$ then using Eq. (64) we obtain $\mathrm{a}_{r}=0.196 \mathrm{eV}$. This magnitude is very close (approximately $84 \%$ ) to the value of $0.235 \mathrm{eV}$ of zero vibration renormalization of the energy band gap in LiH crystals [81]. Using Eq. (65) we obtain $\Delta \mathrm{E}_{g}$ (theor) $=0.134 \mathrm{eV}$ that is very close, on the other hand, to observed experimental value equals $0.103 \mathrm{eV}$. The discrepancy between these values may be caused by the negligible contribution of the isotopic lattice expansion to the band gap renormalization.

By now the change in $\mathrm{E}_{g}$ caused by isotopic substitution has been observed for many broad-gap and narrow-gap compounds. All enumerated above crystals show the dependence of the electronic gap on the isotope masses. It should be noted that the indicated effect (the variation of $\mathrm{E}_{g}$ and $\mathrm{E}_{b}$, see Table 3 and 4 ) have maximum values in $\mathrm{LiH}$ crystals, although this effect in other crystals with isotopic composition are currently being reliably measured and investigated well.

Table 5. Values of the Coefficients $\partial \mathrm{E}_{\mathrm{g}} / \partial \mathrm{M}(\mathrm{meV})$ and Energies of the Band-to-Band Transitions $\mathrm{E}_{\mathrm{g}}(\mathrm{eV})$ according to indicated references (after $[4])$.

\begin{tabular}{|c|c|c|}
\hline Substance & $\partial \mathrm{E}_{\mathrm{g}} / \partial \mathrm{M}(\mathrm{meV})$ & $\mathrm{E}_{\mathrm{g}}(\mathrm{eV})$ \\
\hline${ }^{13} \mathrm{C} \longrightarrow{ }^{12} \mathrm{C}$ & $14.6[1]$ & $5.4125[1]$ \\
\hline${ }^{7} \mathrm{LiH} \longrightarrow{ }^{7} \mathrm{LiD}$ & $103[2]$ & $4.992 \longrightarrow 5.095[2]$ \\
\hline${ }^{7} \mathrm{LiH} \longrightarrow{ }^{6} \mathrm{LiH}$ & $12[2]$ & $4.980[2]$ \\
\hline $\mathrm{CsH} \longrightarrow \mathrm{CsD}$ & $60[3]$ & $4.440[3]$ \\
\hline${ }^{30} \mathrm{Si} \longrightarrow{ }^{28} \mathrm{Si}$ & $2[4]$ & $3.652[4]$ \\
\hline${ }^{30} \mathrm{Si} \longrightarrow{ }^{28} \mathrm{Si}$ & $2.09[5]$ & $1.166[5]$ \\
\hline${ }^{68} \mathrm{ZnO} \rightarrow{ }^{64} \mathrm{ZnO}$ & $0.372[6]$ & $3.400[6]$ \\
\hline $\mathrm{Zn}^{18} \mathrm{O} \rightarrow \mathrm{Zn}^{16} \mathrm{O}$ & $3.533[6]$ & $3.400[6]$ \\
\hline${ }^{68} \mathrm{ZnO} \rightarrow{ }^{64} \mathrm{ZnO}$ & $0.40[7]$ & $3.400[6]$ \\
\hline $\mathrm{Zn}^{18} \mathrm{O} \rightarrow \mathrm{Zn}^{16} \mathrm{O}$ & $3.20[7]$ & $3.400[6]$ \\
\hline${ }^{69} \mathrm{GaP} \longrightarrow{ }^{71} \mathrm{GaP}$ & $0.19[8]$ & $2.400[8]$ \\
\hline${ }^{65} \mathrm{CuCl} \rightarrow{ }^{63} \mathrm{CuCl}$ & $-0.076[9]$ & $3.220[9]$ \\
\hline $\mathrm{Cu}^{37} \mathrm{Cl} \rightarrow \mathrm{Cu}^{35} \mathrm{Cl}$ & $0.364[10]$ & $3.220[10]$ \\
\hline $\mathrm{Cd}^{34} \mathrm{~S} \rightarrow \mathrm{Cd}^{32} \mathrm{~S}$ & $0.370[11]$ & $2.580[11]$ \\
\hline${ }^{110} \mathrm{CdS} \rightarrow{ }^{116} \mathrm{CdS}$ & $0.040 \div 0.068[12]$ & $2.580[11]$ \\
\hline $\mathrm{Cu}_{2}^{18} \mathrm{O} \rightarrow \mathrm{Cu}_{2}^{16} \mathrm{O}$ & $1.116[13]$ & $2.151[13]$ \\
\hline${ }^{71} \mathrm{GaAs} \rightarrow{ }^{69} \mathrm{GaAs}$ & $0.39[9]$ & $1.53[9]$ \\
\hline${ }^{76} \mathrm{Ge} \rightarrow{ }^{72} \mathrm{Ge}$ & $0.225[11-16]$ & $1.53[11-16]$ \\
\hline $76 \rightarrow{ }^{73} \rightarrow{ }^{70} \mathrm{Ge}$ & $0.37[15-16]$ & $0.74[15-16]$ \\
\hline
\end{tabular}

1. T. Collins et al., Phys. Rev. Lett. 65, 891 (1990).

2. V.G. Plekhanov, Uspekhi Fiz. Nauk (Moscow) 167, 577 (1997) (in Russian).

3. K. Chandahari et al. Solid State Commun. 100, 777 (1996) .

4. L.F. Lastras - Martinez et al. Phys. Rev. B61, 12946 (2000) .

5. D. Karaiskaja et al. Solid State Commun. 123, 87 (2002).

6. F.I. Kreingol'd, Fizika Tverdogo Tela 20, 3138 (1978)(in Russian).

7. F.J. Manjon et al. Solid State Commun. 128, 35 (2003).

8. T.A. Meyer et al. Solid State Commun. 126, 119 (2003) .

9. N. Garro et al. Phys. Rev. B54, 4732 (1996) .

10. N. Garro et al. Solid State Commun. 98, 27 (1996).

11. F.I. Kreingol'd and B.S. Kulinkin, Fizika Tverdogo Tela 28, 3164 (1986) (in Russian).

12.T.A. Meyer et al., Phys. Rev. B69, 115214 (2004).

13. F.I. Kreingol'd, Pis'ma v ZETPH 23 (1976) 679; F.I. Kreingol'd, Fizika Tverdogo Tela 27, 2839 (1985) (in Russian).

14. V.F. Agekyan et al. Fizika Tverdogo Tela 31, 101 (1989) (in Russian).

15. C. Parks et al. Phys. Rev. B49, 14244 (1994) .

16. E.E. Haller, J. Appl. Phys. 77, 2857 (1995) .

All of these results on $\mathrm{E}_{g}=\mathrm{f}(\mathrm{x})$ are documented in Table 5, where the variation of $\mathrm{E}_{g}$ and $\partial \mathrm{E}_{g} / \partial \mathrm{M}$ are shown at the isotope effect. We should highlighted here that the most prominent isotope effect is observed in LiH crystals, where the dependence of $\mathrm{E}_{b}=\mathrm{f}\left(\mathrm{C}_{H}\right)$ is also observed and investigated. Using the least-squares method 
it was found the empirical dependence of $\ln \partial \mathrm{E}_{g} / \partial \mathrm{M} \sim \mathrm{f}\left(\ln \mathrm{E}_{g}\right)$, which is depicted on Fig. 26. As can be seen the indicated dependence has a parabolic character:

$$
\ln \left(\partial \mathrm{E}_{g} / \partial M\right)=6.105\left(\ln \mathrm{E}_{g}\right)^{2}-7.870\left(\ln \mathrm{E}_{g}\right)+0.565 \text {. }
$$



Figure 26. the empirical dependence of $\ln \partial \mathrm{E}_{g} / \partial \mathrm{M} \sim \mathrm{f}\left(\ln \mathrm{E}_{g}\right)$

From this figure it can be concluded also that the small variation of the nuclear mass causes the small changes in $\mathrm{E}_{g}$ also. When the nuclear mass increases it causes the large changes in $\mathrm{E}_{g}(\mathrm{C} ; \mathrm{LiH}$; CsH) [151]. Moreover as can be seen from Fig. 26 in last case the empirical dependence $\ln \partial \mathrm{E}_{g} / \partial \mathrm{M} \sim \mathrm{f}\left(\ln _{g}\right)$ is very close to the linear one and in ordinary coordinate system it has a next expression: $\sqrt{\partial E_{g} / \partial M}=\mathrm{E}_{g}^{\sqrt{6.105}}\left(\partial E_{g} / \partial M=\mathrm{E}_{g}^{3.0525}\right)$. By the way it should be noted that at the large changes $\mathrm{E}_{g}$ result in the changes of the force constants at the isotope effect from the large variation of nuclear mass. Observable in Fig. 26 rather large scattering data in the rate of change $\mathrm{E}_{g}$ on the isotope mass in the first step cause the different degree of the isotope-induced-disorder. The last effect, as is well-known (see e.g.[123]), due the different magnitude of the scattering potential at the isotope substitution.

\section{Manifestation of the isotope effect in phonon states}

\subsection{Light scattering}

The simplest kind of motion in solids is the vibrations of atoms around the equilibrium point. The interaction of the crystalforming particles with the one another at the move of the one atom entanglements neighbor atoms [1]. The analysis of this kind motion shows that the elementary form of motion is the wave of the atom displacement. As is well - known that the quantization of the vibrations of the crystal lattice and after introduction of the normal coordinates, the Hamiltonian of our task will be have the following relation (see, e.g. [17])

$$
\mathrm{H}(\mathrm{Q}, \mathrm{P})=\sum_{i, q}\left[-\frac{\hbar^{2}}{2} \frac{\partial^{2}}{\partial Q^{2}(\vec{q})}+\quad \frac{1}{2} \omega_{j}^{2} \mathrm{Q}_{j}^{2}(\vec{q})\right]
$$

In this relation, the sum, where every addend means The Hamiltonian of linear harmonic oscillator with coordinate $\mathrm{Q}_{j}(\vec{q})$, the frequency $\omega_{j}(\vec{q})$ and the mass, which equals a unit. If the Hamiltonian system consists of the sum, where every addend depends on the coordinate and conjugate its quasiimpuls, then according to quantum mechanics [152] the wave function of the system equals the product of wave functions of every appropriate addend and the energy is equal to the sum of assigned energies. Any separate term of the Hamiltonian (67) corresponds, as indicate above, the linear oscillator

$-\frac{\hbar^{2}}{2} \frac{\partial^{2} \Psi}{\partial \mathrm{Q}^{2}}+\frac{1}{2} \omega^{2} \mathrm{Q}^{2} \Psi=\varepsilon \Psi$

Solving last equation and finding the eigenvalues and eigenfunctions and then expressing explicitly the frequency, we will obtain for model with two atoms in primitive cell (with masses $\mathrm{M}_{1}$ and $\mathrm{M}_{2}$ ) the following equation

$$
\begin{aligned}
& \omega^{2} \simeq 2 \mathrm{C}\left(\frac{1}{\mathrm{M}_{1}}+\frac{1}{\mathrm{M}_{2}}\right) \\
& \text { and } \\
& \omega^{2} \simeq \frac{\mathrm{C}}{2\left(\mathrm{M}_{1}+\mathrm{M}_{2}\right)} \mathrm{K}^{2} \mathrm{a}^{2} .
\end{aligned}
$$

Taking into account that $K_{\max }= \pm \pi / a$, where a is a period of the crystal lattice, i.e. $K_{\max }$ respond the border of the first Brillouin zone

$$
\omega^{2}=\frac{2 \mathrm{C}}{\mathrm{M}_{1}} \text { and } \omega^{2}=\frac{2 \mathrm{C}}{\mathrm{M}_{2}}
$$

Formula (98) describes the optical branch of vibrations whereas (70) - acoustical branch of vibrations (see, e.g. Fig. 27). Usually the last formula is written in this way

$$
\omega=\sqrt{\frac{\alpha}{M}} \text {, }
$$




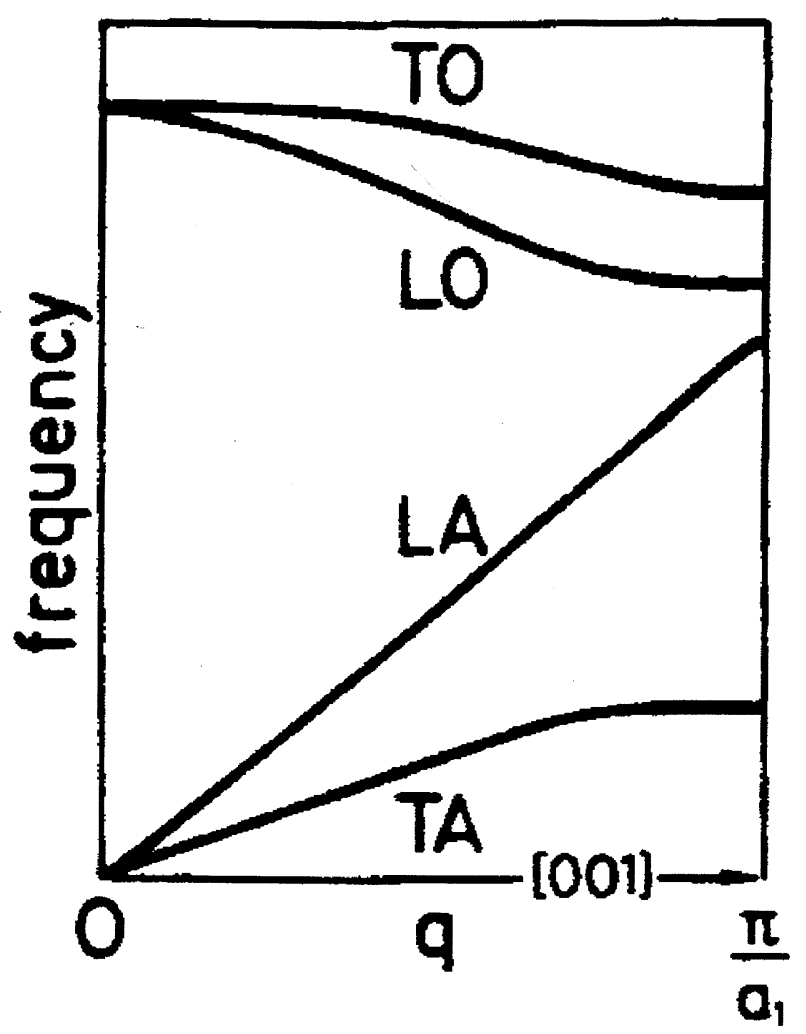

Figure 27. Optical and acoustical modes. The optical modes lie at higher frequencies and show less dispersion than the acoustic modes (for details see text)

where $\alpha$ is so - called the force constant (see, also [1]). Here, as early $\mathrm{M}$ is the mass of vibrated atom (ion). From the preceding relation it is clear that, as in molecular physics, in solid the isotope effect directly manifests in vibration spectrum, which depends on the symmetry [17] measures either in IR - absorption or in Raman scattering of light. Before analyzing Raman scattering spectra of different solids we briefly consider the classical approximation of the mechanism of Raman effect [154 - 157].

Semiconducting crystals (C, Si, Ge, $\alpha$ - Sn) with diamond - type structure present ideal objects for studying the isotope effect by the Raman light - scattering method. At present time this is facilitated by the availability of high - quality crystals grown from isotopically enriched materials (see, e.g [2] and references therein). In this part our understanding of first - order Raman light scattering spectra in isotopically mixed elementary and compound $(\mathrm{CuCl}, \mathrm{GaN}, \mathrm{GaAs})$ semiconductors having a zinc blende structure is described. Isotope effect in light scattering spectra in Ge crystals was first investigated in the paper by Agekyan et al. [ 90]. A more detailed study of Raman light scattering spectra in isotopically mixed Ge crystals has been performed by Cardona and coworkers [2].

It is known that materials having a diamond structure are characterized by the triply degenerate phonon states in the $\Gamma$ point of the Brillouin zone $(\overrightarrow{\mathrm{k}}=0)$. These phonons are active in the Raman scattering spectra, but not in the IR absorption one [158]. Figure $28^{a}$ demonstrates the dependence of the shape and position of the first - order line of optical phonons in germanium crystal on the isotope composition at liquid nitrogen temperature (LNT) [2]. The coordinate of the center of the scattering line is proportional to the square root of the reduced mass of the unit cell, i.e. $\sqrt{\mathrm{M}}$. It is precisely this dependence that is expected in the harmonic approximation. An additional frequency shift of the line is observed for the natural and enriched germanium specimens and is equal, as shown in Ref. [2] to $0.34 \pm 0.04$ and $1.06 \pm 0.04 \mathrm{~cm}^{-1}$, respectively (see, e.g. Fig. 7 in Chapter 4 of Ref. [159]).

Fig. 28. a - First - order Raman scattering spectra Ge with different isotope contents [2] and b - First - order Raman scattering in isotopically mixed diamond crystals ${ }^{12} \mathrm{C}_{x}^{13} \mathrm{C}_{1-x}$. The peaks $\mathrm{A}, \mathrm{B}, \mathrm{C}, \mathrm{D}, \mathrm{E}$ and $\mathrm{F}$ correspond to $\mathrm{x}=0.989 ; 0.90 ; 0.60 ; 0.50 ; 0.30$ and 0.001 (After[158]).

First - order Raman light - scattering spectrum in diamond crystals also includes one line with maximum at $\omega_{L T O}(\Gamma)=1332.5 \mathrm{~cm}^{-1}$. In Fig. $28^{b}$ the first - order scattering spectrum in diamond crystals with different isotope concentration is shown [160]. As shown below, the maximum and the width of the first - order scattering line in isotopically - mixed diamond crystals are nonlinearly dependent on the concentration of isotopes $\mathrm{x}$. The maximum shift of this line is $52.3 \mathrm{~cm}^{-1}$, corresponding to the two limiting values of $\mathrm{x}=0$ and $\mathrm{x}=1$. Analogous structures of first - order light scattering spectra and their dependence on isotope composition has by now been observed many times, not only in elementary $\mathrm{Si}$, and $\alpha-\mathrm{Sn}$, but also in compound $\mathrm{CuCl}$ and $\mathrm{GaN}$ semiconductors (for more details see reviews [1, 2]). Already short list of data shows a large dependence of the structure of first - order light - scattering spectra in diamond as compared to other crystals (Si, Ge). This is the subject detailed discussion in $[20]$. 

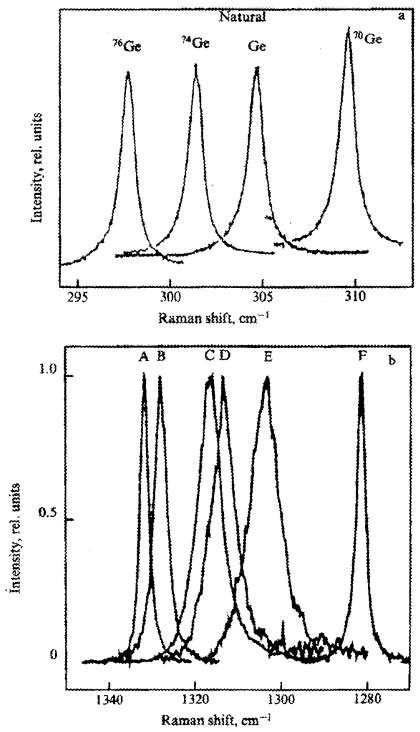

Figure 28. a - First - order Raman scattering spectra Ge with different isotope contents [2] and b - First - order Raman scattering in isotopically mixed diamond crystals ${ }^{12} \mathrm{C}_{x}^{13} \mathrm{C}_{1-x}$. The peaks $\mathrm{A}, \mathrm{B}, \mathrm{C}, \mathrm{D}, \mathrm{E}$ and $\mathrm{F}$ correspond to $\mathrm{x}=0.989 ; 0.90 ; 0.60 ; 0.50 ; 0.30$ and 0.001 (After[160])

Second - order Raman spectra in natural and isotopically mixed diamond have been studied by Chrenko [161] and Hass et al. [162]. Second - order Raman spectra in a number of synthetic diamond crystals with different isotope composition shown in Fig. 29 are measured wit resolution $\left(\sim 4 \mathrm{~cm}^{-1}\right)$ worse than for first - order scattering spectra. The authors of cited work explain this fact by the weak signal in the measurement of second - order Raman scattering spectra. It is appropriate to note that the results obtained in [163] for natural diamond $\left(\mathrm{C}_{13_{C}}=1.1 \%\right)$, agree well with the preceding comprehensive studies of Raman light - scattering spectra in natural diamond [163]. As is clearly seen from Fig. 29 the structure of second - order light scattering "follows" the concentration of the ${ }^{13} \mathrm{C}$ isotope. It is necessary to add that in the paper by Chrenko [161] one observes a distinct small narrow peak above the high - frequency edge of LO phonons and the concentration of ${ }^{13} \mathrm{C} \mathrm{x}=68 \%$. Note is passing that second - order spectra in isotopically mixed diamond crystals were measured in the work by Chrenko [161] with a better resolution than the spectra shown in Fig. 29. Second - order Raman light scattering spectra and IR absorption spectra in crystals of natural and isotopically enriched ${ }^{70} \mathrm{Ge}$ can be found in [1].



Figure 29. Second - order Raman scattering spectra in synthetic diamond with different isotope concentration at room temperature (After[162])

Fig. 29. Second - order Raman scattering spectra in synthetic diamond with different isotope concentration at room temperature (After[164]). 
A comprehensive interpretation of the whole structure of second - order Raman light - scattering spectra in pure $\mathrm{LiH}(\mathrm{LiD})$ crystals is given in [20, 22, 164]. Leaving this question, let us now analyze the behavior of the highest frequency peak after the substitution of hydrogen for deuterium (see, also [166, 132]).

Absorption behavior of an IR - active phonon in mixed crystals with a change in the concentrations of the components can be classified into two main types: one and two - mode (see, e.g. the review [132]). Single mode behavior means that one always has a band in the spectrum with a maximum gradually drifting from one endpoint to another. Two - mode behavior is defined by the presence, in the spectrum, of two bands characteristic of each components lead not only to changes in the frequencies of their maxima, but mainly to a redistribution of their intensities. In principle, one and the same system can show different types of behavior at opposite ends [167]. The described classification is qualitative and is rarely realized in its pure form . The most important necessary condition for the two - mode behavior of phonons (as well as of electrons [168]) is considered to be the appearance of the localized vibration in the localized defect limit. In the review [132] a simple qualitative criterion for determining the type of the IR absorption behavior in crystals with an NaCl structure type has been proposed (see also [168]). Since the square of the TO $(\Gamma)$ phonon frequency is proportional to the reduced mass of the unit cell $\mathrm{M}$, the shift caused by the defect is equal to

$$
\Delta=\omega_{\mathrm{TO}}^{2}\left(1-\frac{\overline{\mathrm{M}}}{\overline{\mathrm{M}}^{\prime}}\right) \text {. }
$$

This quantity is compared in [50] with the width of the optical band of phonons which, neglecting acoustical branches and using the parabolic dispersion approximation, is written as

$$
\mathrm{W}=\omega_{\mathrm{TO}}^{2}\left(\frac{\varepsilon_{0}-\varepsilon_{\infty}}{\varepsilon_{0}+\varepsilon_{\infty}}\right) \text {. }
$$

A local or gap vibrations appears, provided the condition $|\Delta|>(1 / 2) \mathrm{W}$ is fulfilled. As mentioned, however, in [169] in order for the two peaks to exist up to concentrations on the order of $\sim 0.5$, a stronger condition $|\Delta|>$ W has to met. Substituting the numerical values from Tables 1 and 2 of [20] into formulas (73) and (74) shows that for $\mathrm{LiH}(\mathrm{LiD})$ there holds (since $\Delta=0.44 \omega_{\mathrm{TO}}^{2}$ and $\mathrm{W}=0.58 \omega_{\mathrm{TO}}^{2}$ ) the following relation:

$|\Delta|>(1 / 2) \mathrm{W}$.

Thereby it follows that at small concentrations the local vibration should be observed. This conclusion is in perfect agreement with earlier described experimental data. As to the second theoretical relation $\Delta>\mathrm{W}$, one can see from the above discussion that for $\mathrm{LiH}(\mathrm{LiD})$ crystals the opposite relation, i.e. $\mathrm{W}>\Delta$, is observed [167].

Following the results of [21], in Fig. 30 we show the second - order Raman scattering spectra in mixed $\mathrm{LiH}_{x} \mathrm{D}_{1-x}$ crystals at room temperature. In addition to what has been said on Raman scattering spectra at high concentration [22], we note that as the concentration grows further $(\mathrm{x}>0.15)$ one observes in the spectra a decreasing intensity in the maximum of $2 \mathrm{LO}(\Gamma)$ phonons in LiD crystal with a simultaneous growth in intensity of the highest frequency peak in mixed $\mathrm{LiH}_{x} \mathrm{D}_{1-x}$ crystals. The nature of the latter is in the renormalization of $\mathrm{LO}(\Gamma)$ vibrations in mixed crystal [169]. Comparison of the structure of Raman scattering spectra (curves 1 and 2 in Fig. 30) allows us, therefore, to conclude that in the concentration range of $0.1<\mathrm{x}<0.45$ the Raman scattering spectra simultaneously contain peaks of the $\mathrm{LO}(\Gamma)$ phonon of pure $\mathrm{LiD}$ and the $\mathrm{LO}(\Gamma)$ phonon of the mixed $\mathrm{LiH}_{x} \mathrm{D}_{1-x}$ crystal. For further concentration growth $(\mathrm{x}>0.45)$ one could mention two effects in the Raman scattering spectra of mixed crystals. The first is related to an essential reconstruction of the acoustooptical part of the spectrum. This straightforwardly follows from a comparison of the structure of curves 1 - 3 in Fig. 30. The second effect originates from a further shift of the highest frequency peak toward still higher frequencies, related to the excitation of $\operatorname{LO}(\Gamma)$ phonons. The limit of this shift is the spectral location of the highest frequency peak in LiH. Finishing our description of the Raman scattering spectra, it is necessary to note that a resonance intensity growth of the highest frequency peak is observed at $\mathrm{x}>0.15$ in all mixed crystals (for more details see [166]).



Figure 30. Second - order Raman scattering spectra in the isotopically mixed crystals $\mathrm{LiH}_{x} \mathrm{D}_{1-x}$ at room temperature. $1-\mathrm{x}=0 ; 2$ $-0.42 ; 3-0.76 ; 4-1$. The arrows point out a shift of $\mathrm{LO}(\Gamma)$ phonons in the mixed crystals (After [163])

Fig. 30. Second - order Raman scattering spectra in the isotopically mixed crystals $\mathrm{LiH}_{x} \mathrm{D}_{1-x}$ at room 
temperature. $1-\mathrm{x}=0 ; 2-0.42 ; 3-0.76 ; 4-1$. The arrows point out a shift of $\operatorname{LO}(\Gamma)$ phonons in the mixed crystals (After [164]).

Once more reason of the discrepancy between theory and results of the experiment may be connected with not taking into account in theory the change of the force-constant at the isotope substitution of the smaller in size D by $\mathrm{H}$ ion [151]. We should stress once more that among the various possible isotope substitution, by far the most important in vibrational spectroscopy is the substitution of hydrogen by deuterium. As is well-known, in the limit of the Born-Oppenheimer approximation the force-constant calculated at the minimum of the total energy depends upon the electronic structure and not upon the mass of the atoms. It is usually assumed that the theoretical values of the phonon frequencies depend upon the force-constants determined at the minimum of the adiabatic potential energy surface. This leads to a theoretical ratio $\omega(\mathrm{H}) / \omega(\mathrm{D})$ of the phonon frequencies that always exceed the experimental data. Very often anharmonicity has been proposed to be responsible for lower value of this ratio. In isotope effect two different species of the same atom will have different vibrational frequencies only because of the difference in isotopic masses. The ratio $\mathrm{p}$ of the optical phonon frequencies for $\mathrm{LiH}$ and LiD crystals is given in harmonic approximation by:

$$
\mathrm{p}=\frac{\omega(\mathrm{H})}{\omega(\mathrm{D})}=\sqrt{\frac{\mathrm{M}(\mathrm{LiD})}{\mathrm{M}(\mathrm{LiH})}} \simeq \sqrt{2}
$$

while the experimental value (which includes anharmonic effects) is $1.396 \div 1.288$ (see Table1 in Ref. [151]). In this Table there are the experimental and theoretical values of $\mathrm{p}$ according to formula (30), as well as the deviation $\delta=\frac{\mathrm{P}_{\text {Theory }}-\mathrm{p}_{\exp }}{\mathrm{p}_{\text {theory }}}$ of these values from theoretical ones. Using the least squares method it was found the empirical formula of $\ln (\delta \%) \sim \mathrm{f}\left(\ln \left[\frac{\partial \mathrm{E}}{\partial \mathrm{M}}\right]\right)$ which is depicted on Fig. 31. As can be seen the indicated dependence has in the first approximation a linear character:

$$
\ln (\delta \%)=-7.5+2 \ln \left(\frac{\partial \mathrm{E}}{\partial \mathrm{M}}\right) .
$$

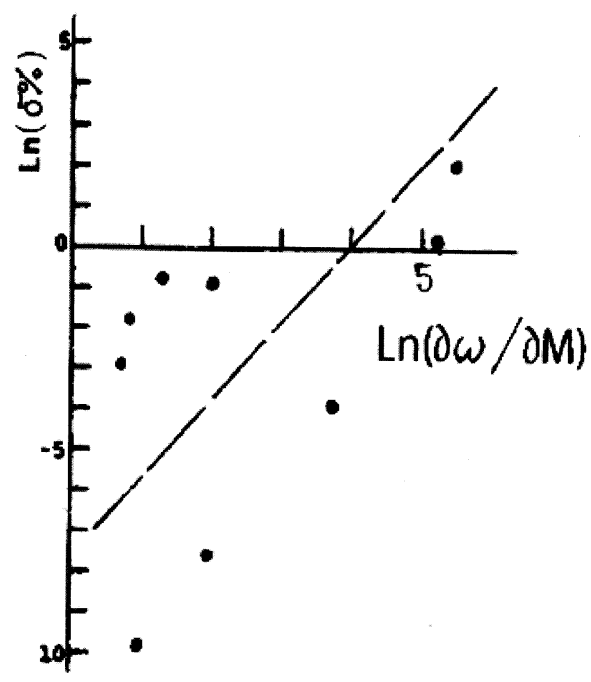

Figure 31. The dependence of $\ln (\delta \%) \sim \mathrm{f}\left[\ln \left(\frac{\partial \omega}{\partial \mathrm{M}}\right)\right]$ : points are experimental values and continuous line - theoretical (After [169])

Fig. 31. The dependence of $\ln (\delta \%) \sim \mathrm{f}\left[\ln \left(\frac{\partial \omega}{\partial \mathrm{M}}\right)\right]$ : points are experimental values and continuous line - theoretical (After [169]).

From the results of Fig. 8, it can be concluded that only hydrogen compounds (and its isotope analog - deuterium) need to take into account the force-constant changes in isotope effect. It is also seen that for semiconductor compounds (on Fig. 31 - points, which is below of Ox line) the isotope effect has only the changes of the isotope mass (for details see [151]).

Thus, the experimental results presented in this section provide, therefore, evidence of, first, strong scattering potential (most importantly, for optical phonons) and, second, of the insufficiency of CPA model for a consistent description of these results [163].

\subsection{Thermal expansion}

It is well-known that in real solids the forces between atoms are not truly harmonic. The higher the temperature, the more phonons that are excited and the more pronounced the effects of anharmonicity (see, e.g. [17]. This leads to changes in the equilibrium separation of the atoms. The lattice therefore expands. Thermal expansion arises as a direct result of the asymmetrical dependence of potential energy on atomic separation, i.e. anharmonicity (see, e.g. [23, 170]). It is therefore not so surprising that the variation of the coefficient of thermal expansion with temperature parallels that of the heat capacity (details see below). When discussing the thermal behavior of solids, it is important to remember that the energy content of the lattice resides in the phonons and not in particular atoms [171]. This may seem paradoxical, but the point is that we cannot convey thermal energy to a particular 
atom (we exclude the case of isolated impurity atoms). We cannot cause an atom to vibrate without exiting a collective oscillation, a phonon [172]. In a pure crystal, and within the harmonic approximation, two or more phonons may pass through one another without interaction. Ar the surface of the sample these phonons would be reflected and continue to exist in unchanged form. But in real solids we find imperfections and anharmonicity, which cause phonons to interact, to be scattered and to decay. Phonons therefore have finite lifetime and we must think of phonon wave packets with particle - like properties. These phonons behave very much like particles in a gas: they constantly interact (collide) and a in a kinetic equilibrium at a given temperature. That this is the case is particularly evident in the thermal conductivity (see below).In insulators and semiconductors (non - metals) heat is conducted solely by the phonons and one might expect thermal energy to be transported at a speed corresponding roughly to that of sound. This may be the case in nearly perfect single crystals at very low temperatures $[173,174$, 175]. However, the mutual interactions of phonons lead to mean free paths of order $100 \AA$, and the phonon gas therefore conducts heat in a similar manner to an ordinary gas. The energy diffuses through the gas and this is a slow process. We should remind, that Debye, long before the concept of the phonon was developed, applied the results of simple kinetic gas theory to solids with remarkable qualitative success [176].

Ubbelohde [153] pointed out in 1936 that the substitution of one isotope for another, in a solid chemical compound, will lead to differences in the rotational and vibrational frequencies of the molecules, without producing any significant change in the structure of the potential energy of the solid. These substitutions cause changes in the lattice constant, a, and in the coefficient of thermal expansion, $\alpha$, of the solid. The influence of isotopic composition on the thermodynamic properties of substances is usually considered to be manifestation of the quantum nature of matter. At high temperatures in the classical limit any mass effect on thermodynamic quantities disappears and hence any quantum isotopic effect vanishes (see, also Landau and Lifshitz [176]). The dependence of the thermal expansion coefficient $\alpha$ from the temperature $\mathrm{T}$ in many cases is dictated by the temperature behavior of the specific heat (see below). It is explained that usually the integral coefficient of Grüneisen $\gamma$ is weak dependence from T. There have been several investigations of the isotope effect on the lithium hydride $[177,179,180,185])$, $\mathrm{KCN}[181]$ and Ge [178, 182, 184].

Table 6. $\alpha, \mathrm{s} \cdot 10^{6}$ for isotopic $\mathrm{LiH}$ (After [181])

$\begin{array}{llll}\text { Isotopic composition } & -190-25^{0} \mathrm{C} & 25-140^{0} \mathrm{C} & 140-240^{0} \mathrm{C} \\ { }^{6} \mathrm{LiH} & 21.0 \pm 0.3 & 34.4 \pm 0.8 & 50.0 \pm 1.0 \\ { }^{7} \mathrm{LiH} & 19.8 \pm 0.4 & 37.4 \pm 0.4 & 53.3 \pm 0.6 \\ { }^{6} \mathrm{LiD} & 24.0 \pm 1.0 & 38.4 \pm 1.8 & 54.3 \pm 1.0 \\ { }^{7} \mathrm{LiD} & 24.8 \pm 0.4 & 42.9 \pm 1.0 & 55.0 \pm 1.4 \\ { }^{7} \mathrm{LiT} & 26.4 \pm-.5 & & \end{array}$

Table 6 lists the measured coefficient of thermal expansion of $\mathrm{LiH}$ and its isotope analog over the temperature ranges $-190^{\circ}-240^{\circ} \mathrm{C}$ [181]. Here, contrary to the effect found with lattice constants (for more details see below) the heavier isotopes have the larger coefficient of thermal expansion. Table 7 lists the ratios of the $\alpha^{\prime}$ s for these same temperature intervals. Several points should be emphasized about table 6 and Table 7 . There is a definite discrepancy in $\alpha$ for ${ }^{6} \mathrm{LiH}$ compared to $\alpha$ for ${ }^{7} \mathrm{LiH}$ in the $-190^{0}$ to $25^{0} \mathrm{C}$ range. There is no reasonable explanation for the higher value of ${ }^{6} \mathrm{LiH}$ over ${ }^{7} \mathrm{LiH}$ (see also [186]). It would be expected that the difference would be small due to the closeness of the reduced masses $(\mu)$ of ${ }^{6} \mathrm{LiH}$ and ${ }^{7} \mathrm{LiH}$; however, the reversal appears to be anomalous.

Table7. Ratio of $\alpha, \mathrm{s}$ for isotopic LiH (After [181]).

\begin{tabular}{|c|c|c|c|}
\hline Ratio & $-190-25^{0} \mathrm{C}$ & $25-140^{\circ} \mathrm{C}$ & $140-240^{\circ} \mathrm{C}$ \\
\hline$\frac{\alpha_{\mathrm{H}}}{\alpha_{\mathrm{D}}}$ for ${ }^{6} \mathrm{Li}$ & 0.879 & 0.896 & 0.921 \\
\hline$\frac{\alpha_{\mathrm{H}}}{\alpha_{\mathrm{D}}}$ for ${ }^{7} \mathrm{Li}$ & 0.805 & 0.872 & 0.969 \\
\hline$\frac{\alpha\left({ }^{6} \mathrm{Li}\right)}{\alpha\left({ }^{7} \mathrm{Li}\right)}$ for $\mathrm{H}$ & 1.060 & 0.920 & 0.982 \\
\hline$\frac{\alpha_{\mathrm{H}}}{\alpha_{\mathrm{T}}}$ for ${ }^{7} \mathrm{Li}$ & 0.750 & & \\
\hline$\frac{\alpha_{\mathrm{D}}^{\mathrm{D}}}{\alpha_{T}}$ for ${ }^{7} \mathrm{Li}$ & 0.932 & & \\
\hline
\end{tabular}

Table 7 shows that for any given combination the ratio $\alpha_{\text {light }} / \alpha_{\text {heavy }}$ approaches 1 as the temperature is increased. Again, all ratios should be exactly one if no isotope effect were present. The greater the relative mass differences between isotopes under study, the lower the ratio $\alpha_{\text {light }} / \alpha_{\text {heavy }}$.

This is especially noticeable in comparing the data for ${ }^{6} \mathrm{Li}$ and ${ }^{7} \mathrm{Li}$ it is seen the ratios are all close to one, indicating very little effect. Theoretical estimation the ratio $\alpha_{\mathrm{LiH}} / \alpha_{\mathrm{LiD}}$ at $298 \mathrm{~K}$ is 0.80 and experimentally, the value $\alpha_{\mathrm{LiH}} / \alpha_{\mathrm{LiD}}$ for the $-190-25^{0} \mathrm{C}$ was 0.81 . This value approaches 1.0 at the higher temperatures. The Debye theory predicts a larger heat capacity for the heavier element $\left(\mathrm{C}_{\text {heavy }}>\mathrm{C}_{\text {light }}\right)$ then the Grüneisen relation $\alpha$ $=\gamma \mathrm{C}_{\mathrm{V}} / 3 \chi_{\mathrm{T}} \mathrm{V}$; it is expected that $\alpha_{\text {heavy }}>\alpha_{\text {light }}$. This difference in physical properties resulting from isotopic substitution becomes much less pronounced as the temperature is increased, and should eventually become negligible (see, also Kogan [187]). These results are shown on the Fig. 32. For comparison, on the Fig. 32 it is shown the results of measurements of the linear thermal expansion coefficients for two samples Ge: ${ }^{\text {nat }} \mathrm{Ge}$ and ${ }^{70} \mathrm{Ge}(99.99 \%)$ [178]. It can be seen from this figure, that the difference $\Delta \alpha$ for two samples Ge one order less than $\Delta \alpha$ in case of $\mathrm{LiH}$ and $\mathrm{LiD}$. In conclusion of this part we should mentioned once more the results of measurements of $\Delta \alpha$ for $\mathrm{KCN}$ of different isotopic composition. The precisely measurements are shown no evidence of influence of isotopic composition on the thermal expansion coefficient or the compression isotherms were found [181]. 




Figure 32. The temperature dependence of the factor $\Delta \alpha=\alpha_{c}\left(\mathrm{M}_{\mathrm{c} 1}\right)-\alpha_{c}\left(\mathrm{M}_{\mathrm{c} 2}\right)$, where $\mathrm{M}_{\mathrm{c} 1}=72.59\left({ }^{\text {nat }} \mathrm{Ge}\right)$ and $\mathrm{M}_{\mathrm{c} 2}=70\left({ }^{70} \mathrm{Ge}\right)$. The full line is the theory, and dots are experiments (After [179])

Fig. 32. The temperature dependence of the factor $\Delta \alpha=\alpha_{c}\left(\mathrm{M}_{\mathrm{c} 1}\right)-\alpha_{c}\left(\mathrm{M}_{\mathrm{c} 2}\right)$, where $\mathrm{M}_{\mathrm{c} 1}=72.59$ (nat $\left.\mathrm{Ge}\right)$ and $\mathrm{M}_{\mathrm{c} 2}=70\left({ }^{70} \mathrm{Ge}\right)$. The full line is the theory, and dots are experiments (After [178]).

Specific heat data are probably the oldest source of in formation about phonons in crystal lattices and were, in fact one of sources of the original paradoxes that led to the development of modern quantum theory. The nonconstancy of the specific heats of crystals as a function of temperature was not explicable by classical theory [17]. The term specific heat is customarily used as a synonym for heat capacity per gram. In most theoretical calculations the heat capacity per gram molecule is the natural quantity to calculate, since this then refers to the properties of a fixed number of particles.

The early low - temperature specific heat was measured by Gūnther from room temperature down to $74 \mathrm{~K}$ [188]. First detailed measurements of the specific heat of lithium hydride in a wide temperature range from 3.7 to 295 were performed by Kostryukov in 1961 [189]. In this paper, the unusual behavior of specific heat was observed between 10 and $20 \mathrm{~K}$. As is shown above, at low temperatures, the heat capacity at a constant volume is $\mathrm{C}_{\mathrm{v}} \sim$ $\gamma \mathrm{T}^{3}$. Kostryukov observed strong deviations from the $\mathrm{T}^{3}$ law, which were dependent on the sample prehistory, and explained them by the presence of free molecular hydrogen in lithium hydride. However, by using a special experimental procedure, Kostryukov managed to obtain more reproducible results that only slightly deviated from the $\mathrm{T}^{3}$ dependence. Based on these results, cited author estimated the Debye temperature of lithium hydride to be $\theta_{\mathrm{D}}=860 \mathrm{~K}$ (see Table 2 in review of Plekhanov [167]). Later, Yates et al. [191] carefully studied the lowtemperature $(5<T<320 \mathrm{~K})$ specific heat of lithium hydride. In this paper, the effect of the isotopic substitution $(\mathrm{H} \rightarrow \mathrm{D})$ on the specific heat was studied for the first time (see Fig. 33). Yattes et al., high-purity and highstoichiometric samples were investigated (the total content of impurities $<2 \times \mathrm{IO}^{-5}$ ). The $\mathrm{C}_{\mathrm{p}}(\mathrm{T})$ dependence measured by Yates et al. at $\mathrm{T}>30 \mathrm{~K}$ was used for calculating the Debye temperature from the relation $\mathrm{C}_{\mathrm{v}}=$ const. $\left(\mathrm{T} / \theta_{\mathrm{D}}\right)^{3}$. The value of $\theta$ determined in this way, as was shown above, depends on the temperature. It is known that this dependence is explained by the deviation of the postulated Debye spectrum from a real phonon spectrum. The values of $\theta_{\mathrm{D}}$ found from the extrapolation of $\theta_{\mathrm{D}}(\mathrm{T})$ to $\mathrm{T}=0$ (Fig. 33) are equal to $\theta_{\mathrm{D}}(\mathrm{LiH})=$ $1190 \pm 80$ and $\theta_{\mathrm{D}}(\mathrm{LiD})=1030 \pm 50 \mathrm{~K}$. One can see that the value of the Debye temperature for LiH obtained by Yates et al. is higher than that obtained by Kostryukov. In addition, data on the Debye temperature show that this temperature decreases with increasing isotope mass in accordance with the theory. Comparison of Debye temperatures calculated from elastic constants $\left(\theta_{\mathrm{D}}=1083-1135 \mathrm{~K}\right.$ at $\left.300 \mathrm{~K}\right)[191,192,193]$ and calorimetric data $\left(\theta_{\mathrm{D}}=1190 \pm 80 \mathrm{~K}\right.$ at $\left.0 \mathrm{~K}\right)[190]$ shows that they are in agreement, especially for LiH crystals. Note the strong temperature dependence of the Debye temperature of LiD crystals, which has not yet been adequately explained. It is likely that the Debye temperature of diamond also strongly depends on temperature (for details see, also $[17,22])$. Fig. 20 displays interesting features in the results below $25 \mathrm{~K}$.Associating the term anomaly with a situation in which the heat capacity rises with diminishing temperatures, the results for ${ }^{7} \mathrm{LiH}$ and ${ }^{7} \mathrm{Lid}$ will each be seen to contain anomalies, having peaks at temperatures of $11.1 \pm 0.2 \mathrm{~K}$ and $12.8 \pm 0.2 \mathrm{~K}$ respectively. The results of Fig. 34 can be little doubt that the peaks observed in the specific heats of the two compounds at low temperatures do not arise from spurious origins, and that they are truly characteristic of the compounds. When they occur in dielectric crystals, anomalies of this type are most commonly caused by movements of atoms, and in this case it seems likely that such a rearrangement takes place in the region of $11.1 \mathrm{~K}$ in the case of ${ }^{7} \mathrm{LiH}$ and in the region of $12.8 \mathrm{~K}$ in the case of ${ }^{7} \mathrm{LiD}$. According cited authors [190] observed $\lambda$ - type anomaly of the heat capacity and concluded that this anomaly in the temperature dependence of $\mathrm{C}_{\mathrm{p}}(\mathrm{T})$ is related to some phase transition. However numerous experimental studies on reflection spectra in exciton region and Raman spectra [168, 194] in these crystals did not reveal noticeable anomalies in the above temperature range (for details see [1]).

Fig. 33. The temperature dependence of the Debye temperature $\theta_{D}$ for ${ }^{7} \mathrm{LiH}$ (a) and ${ }^{7} \mathrm{LiD}$ (b) (After [ 190]).

Fig. 34. The specific heat $\mathrm{C}_{P}$ of ${ }^{7} \mathrm{LiH}(\circ)$ and ${ }^{7} \mathrm{LiD}(\bullet)$ at low temperature (After [190]). 

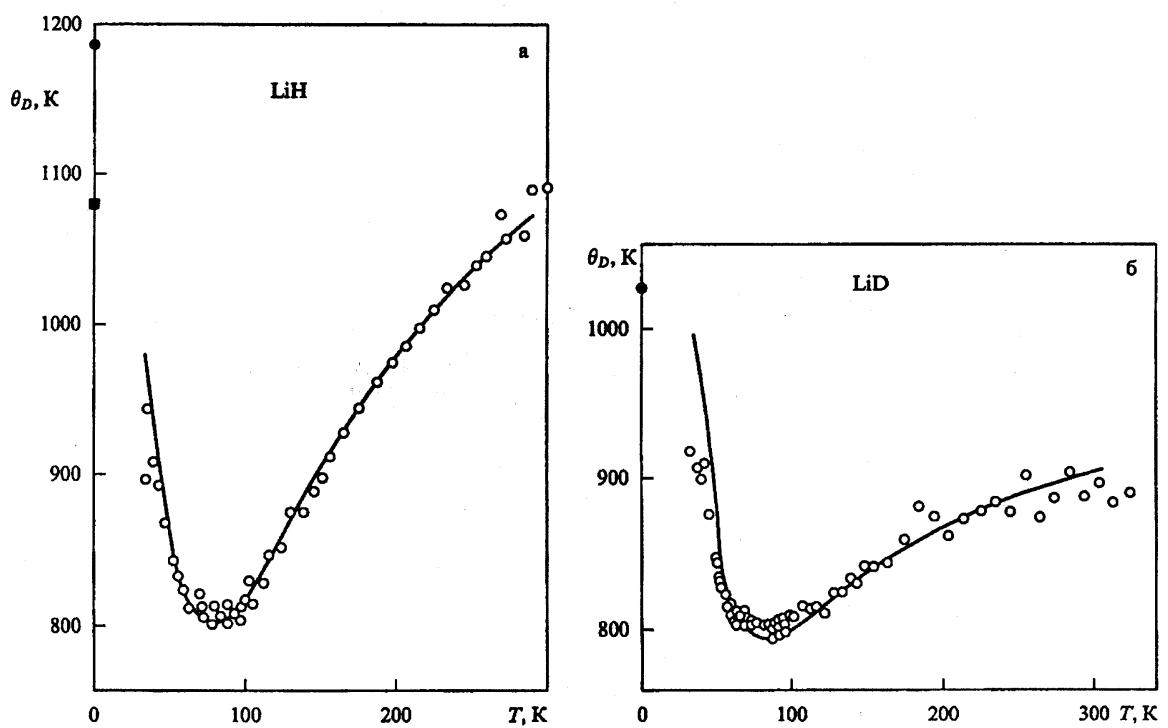

Figure 33. The temperature dependence of the Debye temperature $\theta_{D}$ for ${ }^{7} \mathrm{LiH}$ (a) and ${ }^{7} \mathrm{LiD}$ (b) (After [ 188])



Figure 34. The specific heat $\mathrm{C}_{P}$ of ${ }^{7} \mathrm{LiH}(\circ)$ and ${ }^{7} \mathrm{LiD}(\bullet)$ at low temperature (After [188]) 
In recent years an analogous investigations were performed on semiconducting crystals (see, e.g. [196, 197]). Fig. 35 displays the molar heat capacity of the three investigated Ge isotope samples, again in a plot of $\mathrm{C}_{\mathrm{p}}(\mathrm{T}) / \mathrm{T}^{3}$ against $\mathrm{T}$ (Fig. 35, lower panel), as well as in the representation of the corresponding calculated Debye temperature $\theta_{\mathrm{D}}$ as a function of temperature $\mathrm{T}$ (upper panel). Molar heat capacity and Debye temperature show the characteristic low temperature behavior known from Ge and other solids (see [197] and references therein). Most typical is the strong minimum of $\theta_{\mathrm{D}}(\mathrm{T})$ around $20 \mathrm{~K}$, as a typical for diamond structure, due to the serious departure from the Debye approximation. Anharmonic effects become important only for $\mathrm{T}>\theta_{\mathrm{D}}(0) / 3$, where $\theta_{\mathrm{D}}(\mathrm{T})$ saturates and starts to decrease with increasing $\mathrm{T}$ [196]. Calculations for the difference $\Delta \mathrm{C}_{\text {isotope }}$ between the values of molar heat capacity of the isotopes show that $\Delta \mathrm{C}_{\text {isotope }}$ increases with temperature since $\mathrm{C}_{p}(\mathrm{~T})$ increases most strongly for the material with the greatest molar mass (compare, please with LiH data). $\Delta \mathrm{C}_{\text {isotope }}$ reaches a maximum around $\theta_{\mathrm{D}}(0) / 4(\approx 90 \mathrm{~K}$ for $\mathrm{Ge})$ and at the limit of high temperatures, it approaches zero [17, 196].

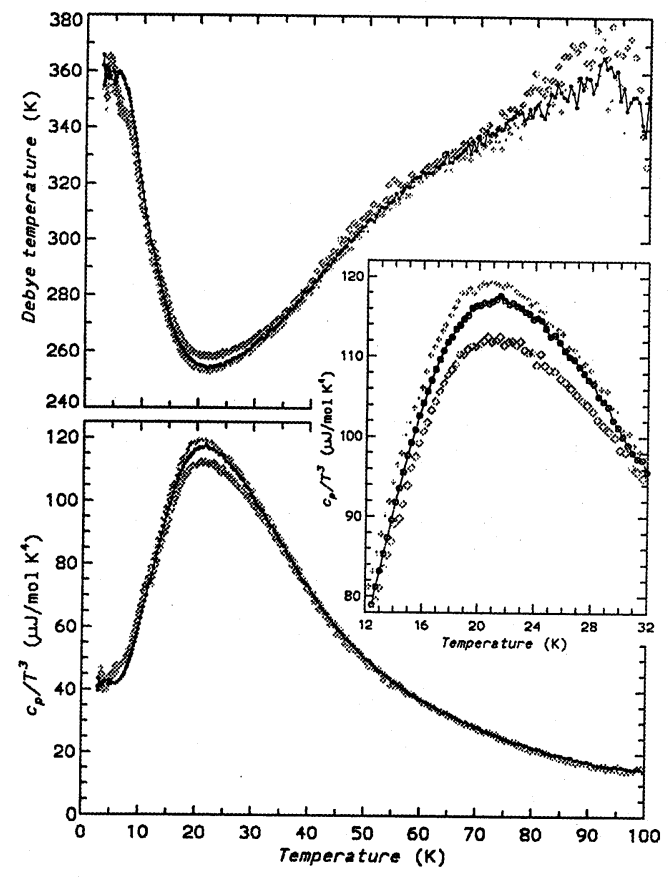

Figure 35. Molar heat capacity $\mathrm{C}_{P}(\mathrm{~T})$ of the three measured crystal samples: ${ }^{70 / 76} \mathrm{Ge}(+)$, ${ }^{\text {nat }} \mathrm{Ge}($ circles connected by a thin line) and ${ }^{70} \mathrm{Ge}(\diamond)$ : plot of $\mathrm{C}_{P} / \mathrm{T}^{3}$ against $\mathrm{T}$ (lower panel), and (upper panel corresponding Debye temperature $\theta_{D}$ as a function of temperature T. The insert shows a magnification of the plot in the lower panel (After [196])

Fig. 35. Molar heat capacity $\mathrm{C}_{P}(\mathrm{~T})$ of the three measured crystal samples: ${ }^{70 / 76} \mathrm{Ge}(+)$, ${ }^{\text {nat }} \mathrm{Ge}$ (circles connected by a thin line) and ${ }^{70} \mathrm{Ge}(\diamond)$ : plot of $\mathrm{C}_{P} / \mathrm{T}^{3}$ against $\mathrm{T}$ (lower panel), and (upper panel corresponding Debye temperature $\theta_{D}$ as a function of temperature $\mathrm{T}$. The insert shows a magnification of the plot in the lower panel (After [196]).

\subsection{Thermal conductivity}

In insulators and semiconductors (at $\mathrm{T}<\theta_{D}$ ) the thermal conduction is effected by phonons, predominantly acoustic ones [175]. Thermal conductivity of crystals has been subject of many experimental theoretical studies (see, e.g. reviews and monographs [175, 198 - 206]). The first experimental results (see, e.g. [206]) have already pointed out the existence of maximum of the thermal conductivity coefficient $\mathrm{k}_{m}$ at about $\mathrm{T} \approx 0.05 \theta_{D}$, where $\theta_{D}$ is the Debye temperature. The growth of $\mathrm{k}$ at low temperatures has been related to phonon scattering due to Umklapp ( $\mathrm{U}_{-}$) - type processes [198]. In the vicinity of $\mathrm{k}_{m}$ thermal conductivity is quite sensitive to impurities and defects in the specimen. The scattering of phonons dynamic isotope disorder is independent of temperature and lattice anharmonicity. The role of isotopes as an additional channel of phonon scattering and their influence on thermal conductivity were first theoretically studied by Pomeranchuk [200] in 1942, and were experimentally studied using Ge in 1958 [201]. According to the results of the latter reference, for a Ge specimen (having 95.8\% ${ }^{74} \mathrm{Ge}$ ), a threefold growth of the thermal conductivity coefficient as compared to the specimen of germanium with natural isotope composition was observed. Later, the influence of isotopes on diamond thermal conductivity was studied many times [206 - 208].

It is generally assumed (see, e. g. [1]) that at not too high temperatures, the dominant interacting among phonons involve three phonons. In a "normal" ( $\left.\mathrm{N}_{-}\right)$process the wave vectors $\vec{q}$ of the phonons are conserved and such process tend to restore a disturbed phonon distribution to one which can be described as a displaced Planck 
distribution (see, e.g. Fig. 5.2 in [17]) which is unaffected by N - processes and corresponds to a heat flow. By themselves, therefore, $\mathrm{N}$ - processes would not lead to a thermal resistance.

In Umklapp (U -) process [202] the wave vectors are not conserved and, as in other resistive processes, they tend to restore a disturbed phonon distribution to the equilibrium Planck distribution which corresponds to zero heat flow, and thus lead to a finite conductivity (for more details, see, review [1]). The Debye expression [175] for the conductivity $\mathrm{k}(\mathrm{T})$ is derived from an adoptional of the simple kinetic theory

$\mathrm{k}(\mathrm{T})=\frac{1}{3}\left\langle\mathrm{v}_{p h}\right\rangle \ell_{p h}(\mathrm{~T}) \mathrm{C}_{p}(\mathrm{~T})$

where $\left\langle\mathrm{v}_{p h}\right\rangle$ is an average phonon velocity, $\ell_{p h}(\mathrm{~T})$ their mean free path and $\mathrm{C}_{p}(\mathrm{~T})$ the corresponding specific heat (for diamond see [202 - 204]). A theory of $\mathrm{k}(\mathrm{T})$ requires basically the calculation of

$\ell_{p h}(\mathrm{~T})=\tau_{p h}(\mathrm{~T})\left\langle\mathrm{v}_{p h}\right\rangle$

a rather formidable task since several scattering mechanisms (normal -, $\mathrm{u}$ - processes, boundary of sample, isotope scattering) [1] contribute to determining the mean free path. In formula $(79) \tau_{p h}(\mathrm{~T})$ is the phonon relaxation time. The simplest of these mechanisms, and the one that can be varied for a given material of the acoustic phonons by isotopic mass fluctuations. This scattering is equivalent to Rayleigh scattering (of photons) at point defect. Within Debye approximation, we will have

$$
\mathrm{k}(\mathrm{T})=\frac{\mathrm{k}_{B}}{2 \pi \nu}\left(\frac{\mathrm{k}_{B}}{\hbar}\right)^{3} \mathrm{~T}^{3} \int_{0}^{\frac{\theta_{D}}{\mathrm{~T}}} \tau(\mathrm{x}) \frac{\mathrm{x}^{4} \mathrm{e}^{x}}{\left(\mathrm{e}^{x}-1\right)^{2}} \mathrm{dx} .
$$

In last expression $\mathrm{k}_{B}$ is the Boltzmann constant. Klemens [174] was the first to try to take the role of $\mathrm{N}$ - processes into account. Using perturbation theory Klemens [172] developed the following expression for the scattering rate $\tau_{\text {isotope }}^{-1}$ :

$$
\tau_{\text {isotope }}^{-1}=\frac{\mathrm{x}(\mathrm{x}-1) \mathrm{V}_{0}}{4 \pi\left\langle\mathrm{v}_{p h}\right\rangle^{3}}\left(\frac{\Delta \mathrm{M}}{\mathrm{M}}\right)^{2} \omega^{2}
$$

where $\mathrm{V}_{0}$ is a volume per atom (for diamond $5 \cdot 7 \cdot 10^{-24} \mathrm{~cm}^{3}$ ) and $\omega$ is phonon frequency. Callaway approach [207] successfully introduces normal phonon scattering $\left(\tau_{N}^{-1}\right)$ and resistive scattering $\left(\tau_{R}^{-1}\right)$ (see formula (4.51) in $[1])$.

In Fig. 36 present the results of Wei et al. [204]. The solid curves are the results of fitting the Callaway theory [207], using a single set of fitting parameter. In this paper, Wei et al. have measured a record thermal conductivity of $410 \mathrm{Wcm}^{-1} \mathrm{~K}^{-1}$ at $104 \mathrm{~K}$ for a $99.9 \%{ }^{12} \mathrm{C}$ enriched diamond. These authors predict that a $99.999 \%{ }^{12} \mathrm{C}$ diamond should have a peak value of thermal conductivity exceeding $2000 \mathrm{Wcm}^{-1} \mathrm{~K}^{-1}$, at about $80 \mathrm{~K}$, assuming, of course, that is not limited by point defect scattering mechanisms other than minority isotopes. Similar results have very recently been reported by Olson et al. [207]. We should stress that none of the currently existing theories accurately takes into account all the possible scattering processes.



Figure 36. Thermal conductivity of natural abundance $\left(1.1 \%\right.$ of $\left.{ }^{13} \mathrm{C}\right)$ diamons (lower squares), isotopically enriched (0.1\% $\left.{ }^{13} \mathrm{C}\right)$ diamond (upper squares), together with the low - temperature data (circles) and high - temperature data. The solid curves are the result of fitting the Callaway theory [201] to the data, using the same set fitting parameters. The inset shows the calculated thermal conductivity corresponding to $1 \%, 0.1 \%$ and $0.001 \%{ }^{13} \mathrm{C}$ concentration according to Callaway theory (After [204])

Fig. 36. Thermal conductivity of natural abundance $\left(1.1 \%\right.$ of $\left.{ }^{13} \mathrm{C}\right)$ diamons (lower squares), isotopically enriched $\left(0.1 \%{ }^{13} \mathrm{C}\right)$ diamond (upper squares), together with the low - temperature data (circles) and high - temperature data. The solid curves are the result of fitting the Callaway theory [201] to the data, using the same set fitting parameters. The inset shows the calculated thermal conductivity corresponding to $1 \%, 0.1 \%$ and $0.001 \%{ }^{13} \mathrm{C}$ concentration according to Callaway theory (After [204]).

Thermal conductivity studies have also performed on very highly enriched, ultra - pure ${ }^{70} \mathrm{Ge}$ (see, reviews $[1$, 2]). The maximum value of $\mathrm{k}_{m}=10.5 \mathrm{kWm}^{-1} \mathrm{~K}^{-1}$ was observed, in the vicinity of $\mathrm{T}=16.5 \mathrm{~K}$, for the ${ }^{70} \mathrm{Ge}$ specimen of $99.99 \%$ purity, which is significantly higher than the value for sapphire $\left(6 \mathrm{kWm}^{-1} \mathrm{~K}^{-1}\right.$ around $\mathrm{T}_{m}$ $=35 \mathrm{~K})$ and comparable to the value for silver $\left(11 \mathrm{kWm}^{-1} \mathrm{~K}^{-1}\right.$ near $\left.\mathrm{T}_{m}=15.4 \mathrm{~K}\right)$. Comparison of experimental results shows [219] that, at its maximum (see, e.g. Fig. $\left.6^{a}[20]\right)$, the thermal conductivity of the ${ }^{70 / 76}$ Ge $(91.91 \%$ ) 
specimen is 14 times less than that of ${ }^{70} \mathrm{Ge}(91.91 \%)$. An increase in $\mathrm{k}$ reaches however, only $30 \%$ at $\mathrm{T}=300 \mathrm{~K}$ (see, also $[1,2])$.

The thermal conductivity of monoisotopic and isotopically mixed specimens of silicon crystals has been studied in following papers [210 - 215]. Since the most detailed results have been obtained by the authors of [210], we restrict ourselves to their consideration. It is well - known that natural silicon consists of three isotopes: ${ }^{28} \mathrm{Si}(\sim$ $92 \%),{ }^{29} \mathrm{Si}(\sim 5 \%)$, and ${ }^{30} \mathrm{Si}(\sim 3 \%)$. The use of monoisotopic silicon (for example ${ }^{28} \mathrm{Si}$ ) can substantially reduce the value of dissipated energy scattered in electronic elements made of silicon (e.g. in the memory of electronic computers [209]). The results studies of the thermal conductivity of monoisotopic and isotopically mixed crystals are shown in Fig. 37. According to the results presented in this Fig. 37, for SI284 specimen $\mathrm{k}^{2}=237(8) \mathrm{Wm}^{-1} \mathrm{~K}^{-1}$ at $300 \mathrm{~K}$, whereas for the SINI (natural $\mathrm{Si}$ ) specimen it is equal to $150 \mathrm{Wm}^{-1} \mathrm{~K}^{-1}$. This means that at $300 \mathrm{~K}$ the thermal conductivity of a monoisotopic ${ }^{28} \mathrm{Si}$ specimen grows, as compared to the natural silicon, by $60 \%$ (later - $10 \%$, see, erratum). At the same time, at about $20 \mathrm{~K}$ (in the vicinity of the maximum of the silicon thermal conductivity curve) $\mathrm{k}$ reaches the value of $30000 \pm 5000 \mathrm{Wm}^{-1} \mathrm{~K}^{-1}$, which is 6 times higher the value $\mathrm{k}=5140$ $\mathrm{Wm}^{-1} \mathrm{~K}^{-1}$ for natural specimen (see, also [199]).

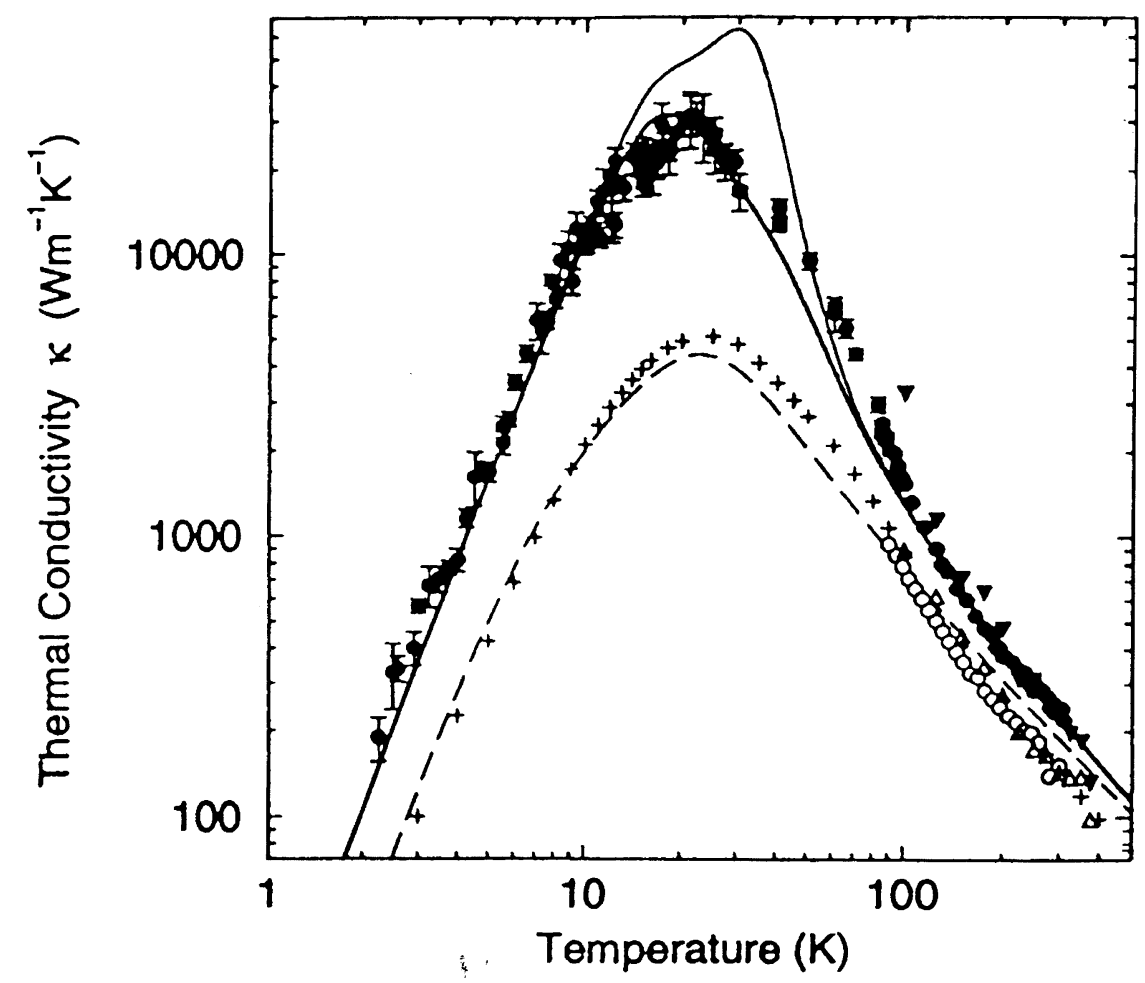

Figure 37. Thermal conductivity of the highly isotopically enriched ${ }^{28}$ Si sample SI284 (filled circles) and the natural Si reference SINI (open circles). The filled and open triangles are other measurements for highly isotopically enriched ${ }^{28} \mathrm{Si}$ and natural Si, respectively (from Ref. [199]); "plus" symbols denote the "standard" curve for natural Si (from [199]). The thin solid and dashed lines are the theoretical results of [212] for ${ }^{28} \mathrm{Si}$ and natural $\mathrm{Si}$, respectively. The thick solid line has been calculated with the same theory using the actual mass variance $\mathrm{g}_{2}$ of sample SI284 (see, also Table 8) (After [214])

Fig. 37. Thermal conductivity of the highly isotopically enriched ${ }^{28} \mathrm{Si}$ sample SI284 (filled circles) and the natural Si reference SINI (open circles). The filled and open triangles are other measurements for highly isotopically enriched ${ }^{28} \mathrm{Si}$ and natural Si, respectively (from Ref. [199]); "plus" symbols denote the "standard" curve for natural Si (from [199]). The thin solid and dashed lines are the theoretical results of [212] for ${ }^{28} \mathrm{Si}$ and natural Si, respectively. The thick solid line has been calculated with the same theory using the actual mass variance $\mathrm{g}_{2}$ of sample SI284 (see, also Table 8) (After [210]).

The thin solid and dashed lines in Fig. 37 correspond to the results of theoretical computations of thermal conductivity for monoisotopic specimen SI284 and for a specimen with natural silicon isotope composition. In these calculations, the model of the Ge thermal conductivity developed in [208] with modified Debye temperature and phonon mean free path has been used. For fitting, the authors have used the low - temperature results, where the thermal conductivity is described by the $\mathrm{T}^{3}$ law. Calculations presented in Fig. 37 were performed, for the natural specimen, for free mean path equals $5.0 \mathrm{~mm}$ (dashed line). For comparison, let us point out that in Ref. [210] the analogous quantity was equal to $5.7 \mathrm{~mm}$, and for isotopically pure SI284 specimen the corresponding value was $14.0 \mathrm{~mm}$ (thin solid line). As seen from Fig. 37, there is agreement between theory and experiment, which has also been mentioned by the authors of [214] themselves. They have also pointed out good agreement between their experimental results and calculations made in [213], except for the domain of U - processes. Beside that, Ruf and co - authors have mentioned an unsatisfactory agreement between theory and experiment in the domain of high 
- temperatures $(300-400 \mathrm{~K})$, especially for the specimen with natural isotope composition. They think that this disagreement can occur due to fundamental reasons that require further study. In particular, taking into account the fine structure of the nonequilibrium phonon distribution function could bring theoretical and experimental results much closer. A qualitative comparison of the influence of the isotope effect on the thermal of germanium, silicon and diamond is given in Table 8.

Table 8. Comparison of the effect of isotopic scattering on the thermal conductivity of natural Ge, Si and diamond at $300 \mathrm{~K}$. The $\%$ increase in $\mathrm{k}$ is the increase of the thermal conductivity of the nearly isotopically pure sample compared to the natural sample. The isotope is defined as $g=\sum_{i} f_{i}\left(\frac{\Delta \mathrm{M}_{i}}{\mathrm{M}}\right)^{2}$ (After [211], [213] and [214]).

$\begin{array}{llll} & \% \text { increase in } \mathrm{k} & \theta & \Gamma \cdot 10^{-4} \\ \mathrm{Ge} & 30 & 376 & 5.80 \\ \mathrm{Si} & 60 & 658 & 2.01 \\ \text { Diamond } & 50 & 1860 & 0.76\end{array}$

In concluding we should remark, that until recently all theories on thermal conductivity had a strongly phenomenological flavor, making use of the relaxation time approximation. In recent years, considerable progress towards an ab initio theory has been made [213, 214]. These authors used two - and three - body potentials obtained by fitting phonon dispersion relations and related the anharmonic properties with a single average Grüneisen parameter. In this manner they determined the third - order coupling coefficients for all possible three - phonon combinations. They then solved iteratively the Boltzmann equation for phonon transport without using the relaxation - time approximation. A scattering time must, however, still be used to describe boundary scattering in the lowest temperature region. In this manner they reproduced rather well the thermal conductivities of Ge, Si and diamond and the observed isotope effects (for details see [213, 214]).

\section{Isotpetronics applications}

This chapter reviews the applications of isotopetronics in different modern technologies and science. The application of stable and radioactive isotopes have described quit wide early in my monographs [17, 219]. Here we shall briefly describe isotopetronics application in two very perspective branches: low - dimensional devices as well as lasers and emitted diodes. .

\subsection{Low - dimensional devices}

Nanoscience is not physics, chemistry, engineering or biology - it is all of them. The same we should tell about isotopetronics. The high level of circuit integration in today's silicon technology could not have been achieved with III - V semiconductors. However, from point of view of operating speed, III - V devices show many advantages (see below), mainly due to the high carrier mobility, $\mu$, and lower effective mass of electrons in III - V compounds [220]. As is well - known, carrier mobility in GaAs is about one order of magnitude higher than that of silicon [220]. In fact, the electron velocity in a semiconductor under the effect of an applied external fiel is probably the most representative parameter for the design of high - speed advanced electronic circuits. Fig. 38 shows the maximum operation frequency (in $\mathrm{GHz}$ ) of different modulation - doped field effect transistors (MODFETs) as a function of gate length (in microns) [220]. Due to their particular characteristics, these transistors are also called high electron mobility transistors (HENTs) [222]. For comparison purpose, Fig. 38 also includes typical parameters of silicon metal - oxide - semiconductor field effect transistors (MOSFETs) as well as GaAs MESFETs. On this picture frequency values are given at room temperature $(300 \mathrm{~K})$ although these frequencies are much higher for operation temperature close to $0 \mathrm{~K}$, as a consequence of the increase in mobility at low temperature (see, e.g. [222]). At present MODFET devices with gate lengths about $100 \mathrm{~nm}$ and maximum operation frequencies at room temperature of several hundreds of $\mathrm{GHz}$ are available.

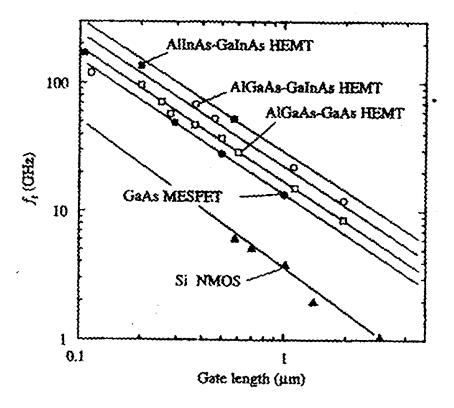

Figure 38. Maximium operation frequencies reached by MODFET, MESFET and MOSFETs as function of gate length (After [224, 225])

Fig. 38. Maximium operation frequencies reached by MODFET, MESFET and MOSFETs as function of gate length (After $\left[221,221^{a}\right]$ ). 
Another very interesting quantum effect to take into account for the development of advanced transistors is the so - called resonant tunneling effect [222]. Resonant tunneling diodes (RTD), based on that effect, basically consist of a quantum well surrounded by two potential barriers thin enough to allow electron tunneling. Due to the extremely low electron transit time through these semiconductors structures, electronic devices based on RTDs can operate at extremely high frequencies, in the range of $1 \mathrm{THz}$. By the addition of an RTD to a bipolar transistor or to a FET, it is possible to built resonant tunneling transistor (RTT). In these transistors, the resonant tunnel structure injects very hot electrons (i.e. electrons of very high kinetic energy) into the transistor active region. The reduction of the characteristic device size to the nanometric range leads to a notable reduction in the number of electrons contained in the electric signals transferred through electronic devices. This tendency has led to the development of the so - called single - electron transistor (SET). As will be seen below, the performance of SETs is based on the Coulomb blockade effect, which is manifested in zero - dimensional semiconductor structures - QDs (see, [4]).

Resonant tunneling diodes. As was shown above that electrons in heterojunctions and in QWs can respond with very high mobility to applied electric fields parallel to the interfaces (see, also [4]). In this part, the response to an electrical field perpendicular to the potential barriers at the interfaces will be considered. Under certain circumstances, electrons can tunnel through these potential barriers, constituting the so - called perpendicular transport (see, also [226]). Tunneling currents through hetero-structures. can show zones of negative differential resistance (NDR) (see, Fig. 38), which arise when the current level decreases for increasing voltage (see [3]). The operation of NDR QW electronic devices is based on the so - called resonant tunnel effect (RTE), which takes place when the current travels through a structure formed by two thin barriers with a QW between them. The I - V characteristics of RTE devices depicted on the Fig. 39. This figure also shows the representation of the conduction band of a double heterojunction with a QW between the junctions. The thickness of the QW is supposed to be small enough $\left(5-10 \mathrm{~nm}\right.$ ) as to have only one allowed energy level $\mathrm{E}_{1}$ (resonant level). The well region is made from lightly doped GaAs surrounded by higher gap AlGaAs (see, e.g. [223]). The outer layers are made from heavily doped $\mathrm{n}$ - type GaAs ( $\mathrm{n}^{+}$GaAs) to facilitate the electrical contacts. The Fermi level of the $\mathrm{n}^{+} \mathrm{GaAs}$ is represented within the conduction band, since it can be considered a degenerated semiconductor [224].

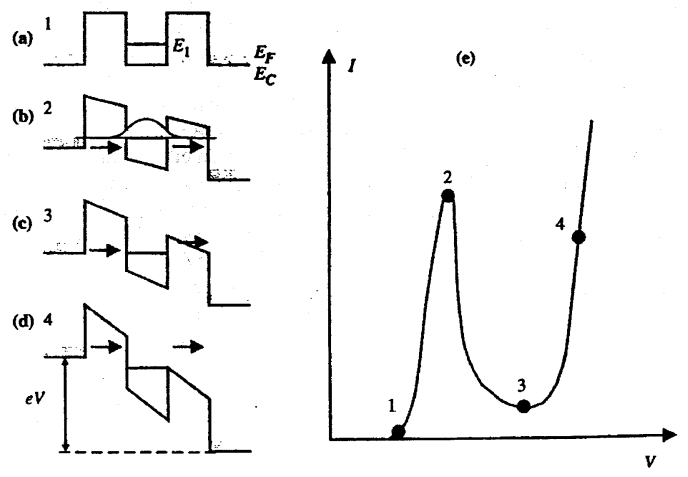

Figure 39. Schematic representation of the conduction band of a resonant tunnel diode:(a) with no valtage, (b - d) for increasing applied voltage, (c) - current - voltage characteristic

Fig. 39. Schematic representation of the conduction band of a resonant tunnel diode:(a) with no valtage, (b d) for increasing applied voltage, (c) - current - voltage characteristic.

Let us suppose that an external voltage, $\mathrm{V}$, is applied, starting from $0 \mathrm{~V}$. It can be expected that some electrons tunnel from the $\mathrm{n}^{+}$GaAs conduction band through the potential barrier, thus resulting in increasing current for increasing voltage (region $1-2$ in the I - V curve of the Fig. 39 ${ }^{c}$ ). When the voltage increases, the electron energy in $n^{+}$GaAs increases until the value $2 \mathrm{E}_{1}$ /e is reached, for which the energy of the electrons located in the neighborhood of the Fermi level coincides with that of level $\mathrm{E}_{1}$ of the electrons in the well (see, Fig. $39^{b}$ ). In this case, resonance occurs and the coefficient of quantum transmission through the barriers rises very sharply. In effect, when the resonant condition is reached, the electron wave corresponding to the electrons in the well is coherently (see, e.g. Fig. 10.18 in [225]) reflected between two barriers. In this case, the electron wave incident from the left excites the resonant level of the electron in the well, thus increasing the transmission coefficient (and thus the current through the potential barrier (region 2 in Fig. $39^{c}$ ). If the voltage further increased (region $2-3$ ), the resonant energy level of the well is located below the cathode lead Fermi level and the current decreases, thus leading to the so - called negative differential resistance (NDR) region (region 2- 3 of the Fig. 39). Finally, for even higher applied voltage, Fig. $38^{d}$, the current again rises due to the thermo ionic emission over the barrier (region 4). RTD used in microwave applications are based on this effect. A figure of merit used for RTD is the peak - to - valley current ratio of their I - V characteristic, given by the ratio between the maximum current (point 2 ) and the minimum current in the valley (point 3). Although the normal values of the figure of merit are about five for AlGaAs - GaAs structures at room temperature, values up to 10 can be reached in devices fabricated from strained InAs layers, surrounded by AlAs barriers and operating at liquid nitrogen temperature [224]. If RTD 
are simulated by a negative resistance in parallel with a diode capacitance $\mathrm{C}$ and a series resistance $\mathrm{R}_{S}$, as is the case of normal diodes, it is relatively easy to demonstrate that the maximum operation frequency increases as $\mathrm{C}$ decreases. The resonant tunnel diode is fabricated from relatively low - doped semiconductors, which results in wide depletion regions between the barriers and the collector region, and accordingly, small equivalent capacity. For this reason, RTDs can operate at frequencies up to several THz, much higher than those corresponding earlier tunnel diodes which just reach about $100 \mathrm{GHz}$, with response time under $10^{-13} \mathrm{~s}$. Small values of the NDR, i.e. an abrupt fall after the maximum on the I - V curve result in high cut - off frequencies of operation. In fact, RTDs are the only purely electronic devices that can operate up to frequencies close to $1 \mathrm{THz}$, the highest of any electron transit time device (see, also [231])

Field effect transistors. The previously analyzed diodes are simplest electronic devices, for which the current is controlled by the diode bias and vice versa. A useful function can be performed mainly due to nonlinearity of current - voltage dependences. In contrast, in three - terminal devices known as transistors there exist the possibility of controlling the current through two electrodes by varying the voltage or the current through third electrode. Below we briefly describe the field effect transistors (FETs) on the base of the nanowires. Nanowire FETs can be configurated by depositing the nanomaterial onto an insulating substrate surface, and making source and drain on the ends nanowire. Fig. 39 illustrates this approach. There, we show a schematic diagram of a Si - nanowire FET with the nanowire, the metal source and drain electrodes on the surface of the $\mathrm{SiO}_{2} / \mathrm{Si}$ substrate (see, also [230]). This approach may serve as the basis for hybrid electronic systems consisting of nanoscale building blocks integrated with more complex planar silicon circuitry [223]. We should note that an extremely small FET may be built on the basis of carbon nanotube [232]. In conclusion of this part we have noted that the nanowire devices discussed here have great potential for applications in nano - and optoelectronics.

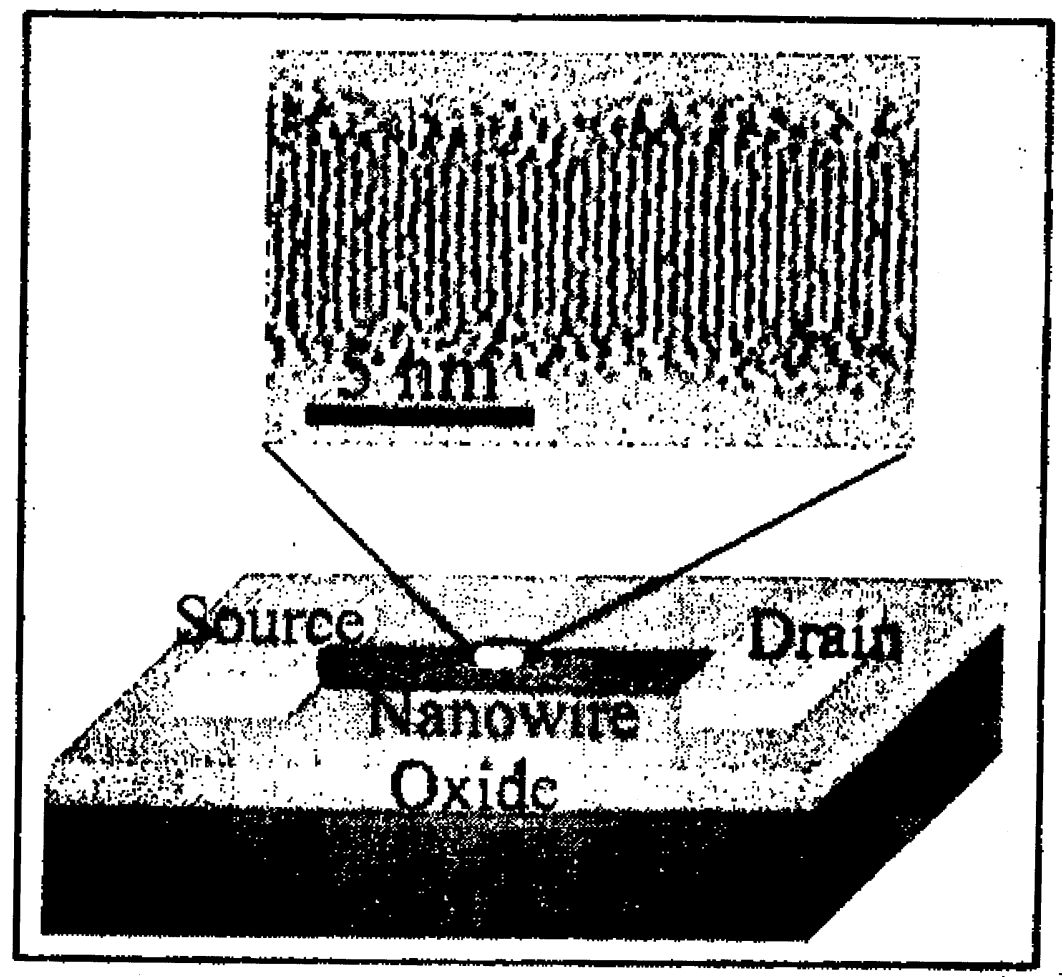

Figure 40. A schematic diagram of a $\mathrm{Si}-\mathrm{FET}$ with nanowire, the metal source, and drain electrodes on the surface of a $\mathrm{SiO}_{2} / \mathrm{Si}$ substrate (After [230])

Fig. 40. A schematic diagram of a Si - FET with nanowire, the metal source, and drain electrodes on the surface of $\mathrm{a} \mathrm{SiO}_{2} / \mathrm{Si}$ substrate (After [225]).

Single - electron - transistor. The so - called single electronics $\quad\left[221,221^{a}, 223,226,227\right.$ 235] appeared in the late 1980s, is at present time a tremendously expanded research field covering future digital and analog circuits, metrological standards, sensors, and quantum information processing and transfer [221]. The basic device, called a single electron device (SED), literally enables the control of electrons on the level of an elementary charge (see, also [230, 231]). There are rich varieties SEDs (see, e.g. [228 - 230]), but the operation principle of all SED is basically the same. SEDs rely on a phenomenon that occurs when electrons are to enter a tiny conducting material (Fig. 41). When the tiny conducting material, or metallic "island", is extremely small, the electrostatic potential of the island significantly increases even when only one electron enters it. For example, for a nanometer scale island having a capacitance $\mathrm{C}$ of, say, $1 \mathrm{aF}\left(10^{-18} \mathrm{~F}\right)$, the increase in the voltage, which is e/C with e $=1.6 \cdot 10^{-19}$ $\mathrm{C}$, reaches $160 \mathrm{mV}$. This is much larger than the thermale noise voltage at room temperature, $25.9 \mathrm{mV}$. Coulomb repulsion prevents additional electrons from entering the island unless the island potential is intentional lowered by an external bias. If the island potential is lowered gradually, the other electrons can enter the island one by one 
with negligibly small power dissipation (for details see $\left[221,221^{a}, 222\right]$ and references therein).

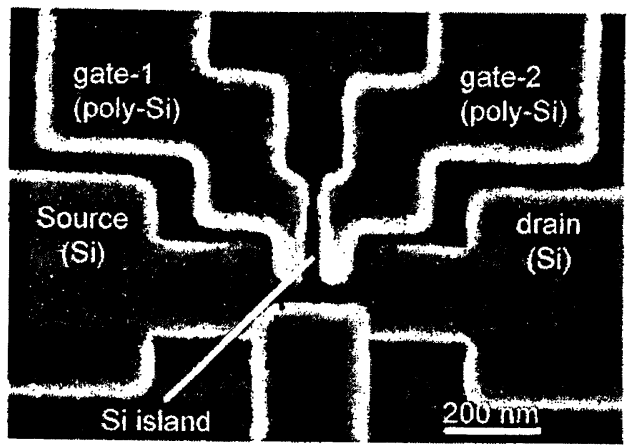

Figure 41. A scanning electron microscope image of a single electron transistor (After [242])

Fig. 41. A scanning electron microscope image of a single electron transistor (After [222]).

The single - electron transistor works as follows. The electron transfer is determined by two factors: the Coulomb charging of the dot and the quantized energy levels in the dot (see above). If the drain is biased with respect to the source, an electric current occurs in the regime of single - electron transfer. By applying the voltage to the gate and changing the QD parameters, one can change the conditions of electron tunneling and affect the source - drain current. Examples of modulation of the conductance in single - electron transistors by the gate voltage are presented in Fig. 42. The devices have almost the same geometry. Their dimensions are large enough to have a number of quantized levels. In Fig. 42 each peak in the conductance corresponds to transfer of one electron, when an energy level enters into resonance with the electron states in the contacts. Though the conductance versus gate - voltage dependences are different, i.e. not reproducible, the peak spacing is the same for both devices. It is determined by the change in the gate voltage required to change the charging energy of the QDs by one electron. The Fig. 42 shows clearly that the electric current is modulated significantly by the gate voltage. Thus, for transistors with single - electron transport, strong control of very small electric current may be possible.

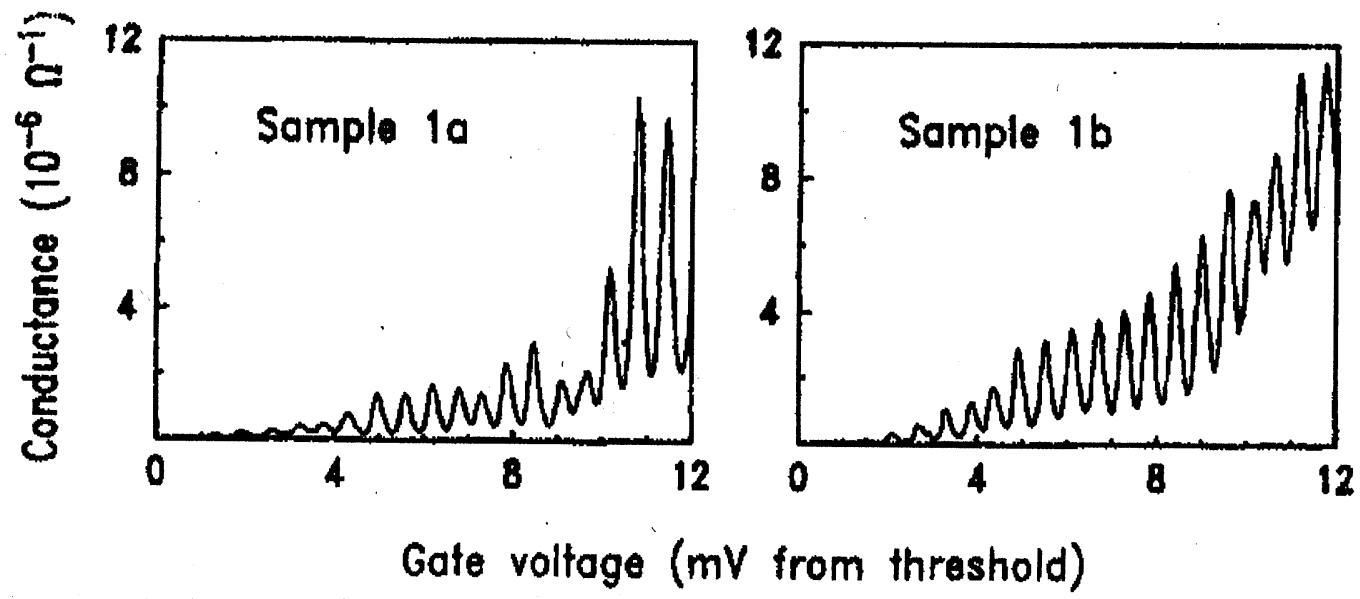

Figure 42. Conductance as a function of $\mathrm{V}_{g}$ for two samples with the same geometry (After [224, 225])

Fig. 42. Conductance as a function of $\mathrm{V}_{g}$ for two samples with the same geometry (After $\left[221,221^{a}\right]$ ).

\subsection{Solid state lasers}

As is well-known, the word laser is an acronym for "light amplification by the stimulated emission of radiation', a phrase which covers most, though not all, of the key physical processes inside a laser. Unfortunately, that concise definition may not be very enlightening to the nonspecialist who wants to use a laser but has less concern about the internal physics than the external characteristics. A general knowledge of laser physics is as helpful to the laser user as a general understanding of semiconductor physics is to the circuit designer. From a practical standpoint, a laser can be considered as a source of a narrow beam of monochromatic, coherent light in the visible, infrared or UV parts of spectrum. The power in a continuous beam can range from a fraction of a milliwatt to around 20 kilowatts $(\mathrm{kW})$ in commercial lasers, and up to more than a megawatt in special military lasers. Pulsed lasers can deliver much higher peak powers during a pulse, although the average power levels (including intervals while the laser is off and on) are comparable to those of continuous lasers 
The range of laser devices is broad. The laser medium, or material emitting the laser beam, can be a gas, liquid, crystalline solid, or semiconductor crystal and can range in size from a grain of salt to filling the inside of a moderate-sized building. Not every laser produces a narrow beam of monochromatic, coherent light. A typical laser beam has a divergence angle of around a milliradian, meaning that it will spread to one meter in diameter after traveling a kilometer. This figure can vary widely depending on the type of laser and the optics used with it, but in any case it serves to concentrate the output power onto a small area. Semiconductor diode lasers, for example, produce beams that spread out over an angle of 20 to $40^{\circ}$ hardly a pencil-thin beam. Liquid dye lasers emit at a range of wavelengths broad or narrow depending on the optics used with them. Other types emit at a number of spectral lines, producing light is neither truly monochromatic nor coherent. Practically speaking, lasers contain three key elements. One is the laser medium itself, which generates the laser light. A second is the power supply, which delivers energy to the laser medium in the form needed to excite it to emit light. The third is the optical cavity or resonator, which concentrates the light to stimulate the emission of laser radiation. All three elements can take various forms, and although they are not always immediately evident in all types of lasers, their functions are essential. Like most other light sources, lasers are inefficient in converting input energy into light; efficiencies can range from under 0.01 to around $20 \%[232,233]$.

Semiconductor lasers, like other lasers, have population inversions which lead to stimulated emission of photons. Semiconductor laser is different from another lasers primarily because the energy levels in semiconductors must be treated as continuous distributions of levels rather than as discrete levels [234]. We shall assume that the densities of states in the conduction and valence bands of the semiconductor are known functions of energy, and that the occupations of these levels are characterized by quasi-Fermi levels [234]. Then the probability that the state of energy $\mathrm{E}$ in the conduction band is occupied by an electron is

$\mathrm{f}_{c}(\mathrm{E})=1 /\left\{1+\exp \left[\left(\mathrm{E}-\mathrm{F}_{c}\right) / \mathrm{kT}\right]\right\}$

where $\mathrm{F}_{c}$ is the quasi-Fermi level for the conduction band, $\mathrm{k}$ is Boltzmann's constant, and $\mathrm{T}$ is absolute temperature. A corresponding expression applies in the valence band, with quasi-Fermi level $\mathrm{F}_{\mathrm{v}}$. For a system in thermal equilibrium, the quasi-Fermi levels are equal to each and become the Fermi level $\mathrm{E}_{F}$. In an excited system we have $\mathrm{F}_{c}>\mathrm{F}_{\mathrm{v}}$, and we can use the separation of the quasi-Fermi levels as a measure of the excitation. The use of quasi-Fermi levels greatly simplifies the treatment of systems with many energy levels, or with continuous distributions of levels, because one quantity represents the occupation probability of many levels. The concept of quasi-Fermi level in an excited system is valid provided the scattering rate of carriers within a band is rapid compared to the recombination rate between bands, i.e. if the carriers within the conduction band and within the valence band rapidly establish a quasi-equilibrium among themselves although the conduction band and the valence band are not in equilibrium with each other For semiconductors with substantial numbers of free carriers, carrier-carrier scattering will lead to the establishment of quasi-equilibrium (see e.g. [235, 236]).

The original semiconductor lasers were p-n junctions prepared by diffusion of acceptor impurities into n-type GaAs, and this is still one of the most common structures. Semiconductors with $\vec{k}$ - conserving transitions at the energy gap are strongly favored for lasing, but some impurity levels can lead to stimulated emission in indirectgap semiconductors, e.g. [237, 238]. All the p-n junction lasers are excited by passing current through the p-n junction, and the excitation rate is characterized by the current density. When a forward current flows, electrons are injected into the p-type material and holes are injected into the n-type material, the latter to a much smaller extent partly because of the lower hole mobility. In heterojunctions, potential barriers play a major role in the injection of carriers [237]. The excess of electron and hole concentrations over their equilibrium values creates a local population inversion and leads to stimulated emission of photons at sufficiently high excitation levels. The layer near the p-n junction where this occurs is called the active region or active layer of the device. Fig. 91. of Ref. [183] shows that the effective thickness of the active layer in graded junction lasers increases as the current density increases. This leads to smaller quasi-Fermi level separations and to less efficient use of the excitation for lasing. Heterostructure lasers (see also [252]) contain built-in potential barriers for the electrons which tend to confine them to regions of fixed width. Thus the excitation can be used most effectively.

A second class of excitation methods involves excitation with photons [241] or with an electron beam [239, 240]. For optical excitation, the active layer thickness will be of the order of $1 / \alpha$, where $\alpha$ is the absorption coefficient of the incident photons. For electron beam excitation, the active layer thickness will be of the order of the penetration depth of the electrons, which is a function of their energy (see, e.g. [228]). In both cases, diffusion of carriers will add a distance of the order of the diffusion length to the thickness given. If sufficiently thin samples are used, the excitation state may be relatively uniform through the sample, provided surface recombination is unimportant. The excitation rate for the externally excited structure can be converted to an equivalent current density. For photons, the absorbed photon flux is multiplied by the electronic charge provided that each absorbed photon give rise to an electron - hole pair. It should be added that values of about $3 \mathrm{E}_{g}$ are necessary to create one e - $\mathrm{h}$ pair [241]. This means that the main part of the incident pump energy is transferred into heat. This is one of the disadvantages of e-beam pumping.

With increasing excitation intensity, frequently laser action is observed in the excitonic luminescence. However, the direct recombination of an exciton can never give rise to laser action, because the coupled exciton-photon system corresponds in the resonant approximation to two linearly coupled harmonic oscillators. The equations of motion of this system do not contain the nonlinearity which is necessary to describe laser action. The participation of a third field is required in order to introduce the possibility of laser action $[229,230]$, i.e. the laser action in 
exciton systems has to be a parametric process in which a pump field, a signal field and an idler field participate.

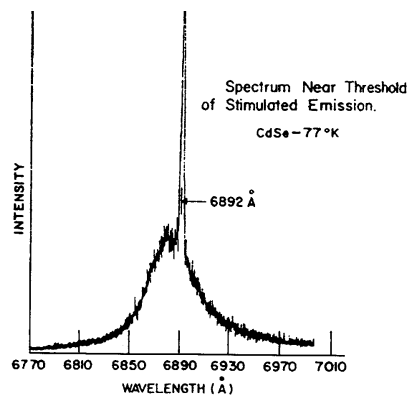

Figure 43. The onset of stimulated emission in CdSe at 77K (After [260])

Fig. 43. The onset of stimulated emission in CdSe at 77K (After [245]).

Below we describe a scheme for lasing action involving excitons in a pure crystal [230, 242]. As was shown above optical transitions in pure III-V compounds which can be used for laser action are band-band transitions. In II-VI compounds (as well as $\mathrm{LiH}$ [183] and etc.), the recombination process of electrons and holes via exciton states is more favorable than the band-band transition [65, 58, 72]. During last four decades laser action has been obtained in II-VI compounds by electron beam bombardment [243 - 247], by optical excitation [243 - 247]. The laser transitions involve the $\mathrm{A}_{1}$ - $\mathrm{nLO}$ phonons, where $\mathrm{n}=1 ; 2$. Gain measurements [58, 248 -250] and simultaneous measurements of the emission intensities of the $\mathrm{A}_{1}$ line (direct $\mathrm{A}$ - exciton recombination [246, 247, 249]) and the $\mathrm{A}_{1}$ - LO line also confirm the statement that in CdS the $\mathrm{A}_{1}$ - LO ( $\left.\mathrm{A}_{1}-2 \mathrm{LO}\right)$ line starts to lase for sufficiently high pump rates (see also Fig. 43) (for details see [260]). In cited papers Haug [229, 230] calculated the temperature dependence of the maximum gain frequency at threshold. The result is simple in the low-temperature limit (see, also [183])

$\Delta_{\max } \rightarrow \frac{3}{2} \mathrm{kT}$

and also in the high-temperature limit

$\Delta_{\max } \rightarrow(3 \kappa \mathrm{kT} / \mathrm{B})^{2 / 5} \exp (-2 \hbar \nu / 5 \mathrm{kT}$,

where $2 \kappa=1.25 \cdot 10^{12} \mathrm{~s}^{-1}$, corresponding to losses of $100 \mathrm{~cm}^{-1}, \mathrm{~B}=1.55 \cdot 10^{35} \mathrm{erg}^{-3 / 2} \mathrm{~s}^{-1}$ for CdS crystals. These limiting results have also been given by Mashkevich et al. [250]. The typical experimental gain $\mathrm{I}_{t h r}(\mathrm{~T})$ dependence, obtained in paper [250] is presented in Fig. 44. There are shown some spectra of stimulated emission at different temperatures. Authors of [250] indicated some contradiction of their experimental results with theoretical description.

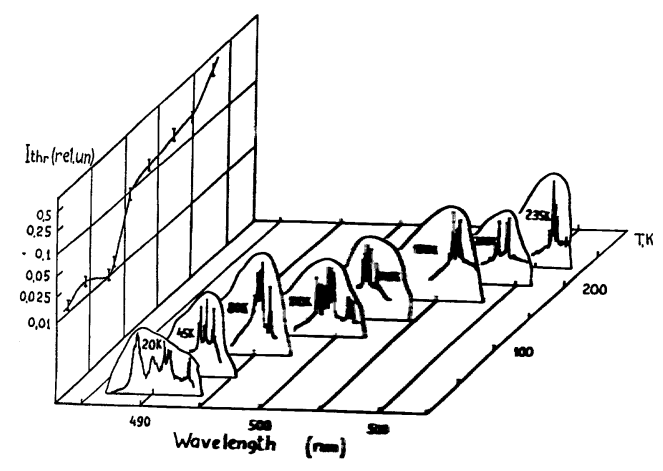

Figure 44. The dependence $\mathrm{I}_{t h r}(\mathrm{~T})$ and some examples of (above-threshold) lasing spectra (in the range of $\mathrm{A}_{1}-1 \mathrm{LO} ; \mathrm{A}_{1}-2 \mathrm{LO}$ phonons) at different temperature (After [265])

Fig. 44. The dependence $\mathrm{I}_{t h r}(\mathrm{~T})$ and some examples of (above-threshold) lasing spectra (in the range of $\mathrm{A}_{1}$ $1 \mathrm{LO} ; \mathrm{A}_{1}-2 \mathrm{LO}$ phonons) at different temperature (After [249]).

Isotope - mixed bulk lasers. As was shown in papers [183, 22, 251] another application of isotope pure and isotope mixed crystals that will be discussed here is related to the possibility of using an isotopically mixed medium (e.g. $\mathrm{LiH}_{x} \mathrm{D}_{1-x}$ or ${ }^{12} \mathrm{C}_{x}{ }^{13} \mathrm{C}_{1-x}$ ) as an oscillator of coherent radiation in the ultraviolet spectral range $[252,253]$. To achieve this, the use of indirect electron transitions involving, say, LO phonons was planned [229, 230]. As was shown above using indirect electron transitions involving phonons to degenerate coherent radiation in semiconductors was originally proposed by Basov et al. (see [244, 245] and reference therein). Kulevsky and Prokhorov [248] were the first to observe stimulated radiation using emission lines of LO phonon repetitions in CdS crystals on two photon excitation (see also [254]). The detection of LO phonon replicas of free - exciton luminescence in wide - gap insulators attracted considerable attention to these crystals (see e.g. [254]). At the same time it is allowed one to pose a question about the possibility of obtaining stimulated emission in UV (VUV) 
region $(4-6 \mathrm{eV})$ of the spectrum, where no solid state sources for coherent radiation exist yet. In the first place this related to the emitters working on the transitions of the intrinsic electronic excitation (exciton). The last one provides the high energetical yield of the coherent emission per unit volume of the substance.

In this part we will discuss the investigation results of the influence of the excitation light density on the resonant secondary emission spectra of the free - exciton in the wide - gap insulator $\mathrm{LiH}_{x} \mathrm{D}_{1-x}\left(\mathrm{LiH}_{1-x} \mathrm{~F}_{x}\right)$ crystals. The cubic $\mathrm{LiH}$ crystals are typical wide - gap ionic insulator with $\mathrm{E}_{g}=4.992 \mathrm{eV}$ [251] with relatively weak exciton - phonon interaction however: $\mathrm{E}_{B} / \hbar \omega_{L O}=0.29$ where $\mathrm{E}_{B}$ and $\hbar \omega_{L O}$ are exciton binding energy and longitudinal optical phonon's energy , respectively. Besides it might be pointed out that the analogous relation for CdS, diamond and $\mathrm{NaI}$ is $0.73 ; 0.45$ and 12.7, respectively [252]. Figure 45 depicts, as an example, the exciton luminescence spectrum of pure $(\mathrm{LiH}$ and $\mathrm{LiD})$ and mixed $\left(\mathrm{LiH}_{x} \mathrm{D}_{1-x}\right)$ crystals at a low temperature. Analogous results for ${ }^{12} \mathrm{C}_{x}{ }^{13} \mathrm{C}_{1-x}$ mixed diamond crystals are shown in Fig. 98 of Ref [183]. A common feature of all three spectra depicted in Fig. 45 is a phononless emission line of free excitons and its 1LO and 2LO phonon replicas. An increase in the density of the exciting light causes a burst of the radiation energy in the long-wave wing of the emission of the $1 \mathrm{LO}$ and $2 \mathrm{LO}$ repetitions at a rate is higher for the $1 \mathrm{LO}$ repetion line [80, 251]. The further investigations have shown [80] that with the increase of the excitation light intensity at the beginning a certain narrowing can be observed, followed by widening of the line of 2LO phonon replica with a simultaneous appearance of a characteristics, probably mode structure. A proximity of the exciton parameters of $\mathrm{LiH}$ and $\mathrm{CdS}(\mathrm{ZnO})$ crystals allowed to carry out the interpretation of the density effects in $\mathrm{LiH}$ on the analogy with these semiconducting compounds. Coming from this in the paper [272] it was shown that for the observed experimental picture on LiH crystals to suppose the excitonphonon mechanism of light generation [242] is enough the excitons density about $10^{15} \mathrm{~cm}^{-3}$. This is reasonable value, if the high quality of the resonator mirrow - the crystal cleavage "in situ" and relatively large exciton radius $(\mathrm{r}=40 \AA[251])$ is taken into account. To this light mechanism generation must be also promoting a large value of the LO phonon energy $\left(\hbar \omega_{L O}=140 \mathrm{meV}\right)$. Owing to this the radiative transition is being realized in the spectral region with a small value of the absorption coefficient, and thus with a small losses in resonator (for details see also [251], see also [22]).

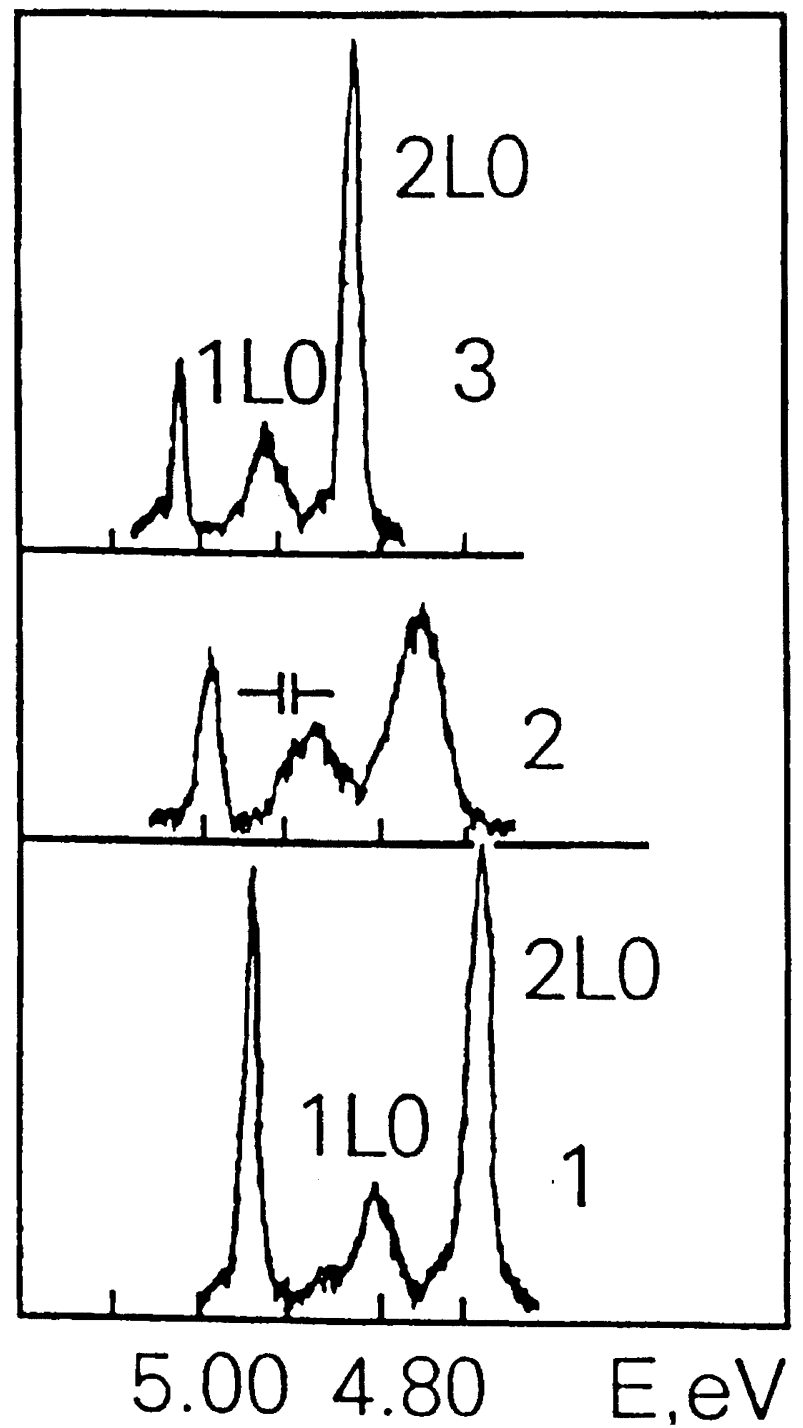

Figure 45. Photoluminescence spectra of free excitons at 4.2K: $1-\mathrm{LiH} ; 2-\mathrm{LiH}_{x} \mathrm{D}_{1-x}$ and $3-\mathrm{LiD}$ crystals (After [271]) 
Fig. 45. Photoluminescence spectra of free excitons at 4.2K: $1-\mathrm{LiH} ; 2-\mathrm{LiH}_{x} \mathrm{D}_{1-x}$ and 3 - LiD crystals (After $[251])$.

Light - emitting diodes and lasers of low - dimensional structures. So far we have studied electronic nanoscale devices, i.e., a class of devices that exploits electrical properties of nanostructures and operates with electric input and output signals. Another class is composed of optoelectronic devices, which are based on both electrical and optical properties of materials and work with optical and electric signals. In this paragraph we will analyze two very important classes of optoelectronic devices: light - emitting diodes and lasers. As will be shown below, the energy of the electric current flowing through these diodes is transformed into light energy. These optoelectronic devices have a huge number of applications and deserve consideration in details (see, also [255 $258])$.

Although stimulated emission [4] from the injection laser diode is very important (see, below), practically, sub threshold operation of the diode - when only spontaneous light is emitted - is in many cases advantageous and has a number of applications. Diodes operating with spontaneous light emission are called light - emitting diodes [237]. The important characteristic of the light - emitting diode is the spectral distribution of emission The spectrum of emission is determined, primarily, by the electron/hole distributions. Thus, the ambient temperature T, defines both spectral maximum and the spectral width of emission. The peak value of the spectral distribution can be estimated as [224]

$$
\hbar \omega=\mathrm{E}_{g}+\frac{\mathrm{kB}_{\mathrm{B}}}{2} \text {. }
$$

The full width at half maximum of the distribution is $\Delta \omega \approx 2 \mathrm{k}_{\mathrm{B}} \mathrm{T} / \hbar$ and is independent of $\omega$. In terms of the wavelength, $\lambda$, we obtain

$$
\Delta \lambda=\left[\lambda_{m}^{2} /(2 \pi c) \Delta \omega\right.
$$

$\Delta \lambda=1.45 \lambda^{2} \mathrm{k}_{\mathrm{B}} \mathrm{T}$

where $\lambda_{m}$ corresponds to the maximum of the spectral distribution, $\Delta \lambda$ and $\lambda_{m}$ are expressed in micrometers, and $\mathrm{k}_{\mathrm{B}} \mathrm{T}$ is expressed in eV. Fig. 46 shows the spectral density as a function of the wavelength for light - emitting diodes based on various materials. For these different materials, the spectral linewidth increase in proportion to $\lambda^{2}$, in accordance with Eq. (86). From Fig.47, one can see that light - emitting diodes cover a wide spectral region from the infrared - about $8 \mu \mathrm{m}$ for InGaAsP alloys - to the near ultraviolet - $0.4 \mu \mathrm{m}$ for GaN. Light - emitting diodes are, indeed very universal light sources [258].

Fig. 46. The dependence of the shape of $2 \mathrm{LO}$ replica line on the excitation intensity $\left(\mathrm{I}_{0}\right)$ light: $1-0.05 \mathrm{I}_{0} ; 2-$ $0.09 \mathrm{I}_{0} ; 3-0.35 \mathrm{I}_{0} ; 4-\mathrm{I}_{0}$ (After [253]).

Fig. 47. The spectra of light - emitting semiconductor diodes with different bandgaps (After [221]).

Semiconductor lasers incorporating low - dimensional hetero-structures., QWs and QDs, are attracting considerable interest of their potential for improved performance over QW lasers (see, e.g. [257, 258]). This prediction is based, in the single - particle picture, on the sharper density of states resulting from the confinement of the charge carriers in two or three directions. Among other advantages, the ideal QD and QWr lasers would exhibit higher and narrower gain spectrum, low threshold currents, better stability with temperature, lower diffusion of carriers to the device surfaces, and a narrower emission line than double heterostructure or QW lasers (see, also [259, 259 $\left.{ }^{a}\right]$ ). The observation of lasing from excitons in optically excited V - groove GaAs/AlGaAs QWr laser structures was detail describe in paper [260]. The observable emission is attributed to the recombinations of excitons associated with the lowest energy electron - and hole - subbbands of the QWr. Moreover these authors show that the emission energy remains nearly constant within the inhomogeneously broadened photoluminescence line of the QWrs for both continuous wave (cw) and pulsed optical excitation over a wide range of power densities. These results corroborate the important role played by electron - hole Coulomb correlations [260, 261] in the optical emission from quasi - 1D QWrs in the density regime of the Mott transition.

Fig. 48. (a) Photoluminescence spectra at $10 \mathrm{~K}$ of the QWr laser sample above, below and near the lasing threshold in TE - polarization. (b) Dependence on input excitation power of the PL output power;arrows indicate the excitation powers used for the optical spectra depicted in (a). (c) High resolution emission spectrum above the lasing threshold showing the Fabri - Perrot modes of the optical cavity (After [260]).

Optical emission of the QWr laser structure are displayed in Fig. 48 for different values of the optical power density below, at and above the threshold for lasing in the QWr. Upon increasing the pump power, these authors observe a nearly constant energy of the peak at $1.581 \mathrm{eV}$ that corresponds to the optical transition $\mathrm{e}_{1}-\mathrm{h}_{1}$ associated with the ground electron - hole - subband of the QWrs. A significant spectral narrowing is also found as the power density is increased and crosses the lasing threshold. This evidences the existence of amplified spontaneous emission within this inhomogeneously broadened PL line in this density regime. The observable emission intensity varies linearly at low excitation power over three orders of magnitude (from 0.1 to $100 \mathrm{~mW}$ ) [278]). Above the lasing threshold (at $350 \mathrm{~mW}$ ) the intensity variation is again linear (see, Fig. $48^{b}$ ), indicating that the modal gain is saturated. In Fig. $48^{c}$, a high - resolution emission spectrum obtained above threshold features well - resolved Fabry - Perot modes that correspond to different longitudinal optical modes within the inhomogeneuous line of the QWr - PL. Detailed investigations of PL and PLE spectra (see, Fig. 49) of the QWr allowed the indicated authors to conclude that the lasing emission originates from the recombination of excitons as it is case for the QWr - peak of the cw - PL spectrum (for details see [260]).

Fig. 49. Linearly - polarized PLE spectrum and the corresponding PL spectrum of an etched QWr laser sample 


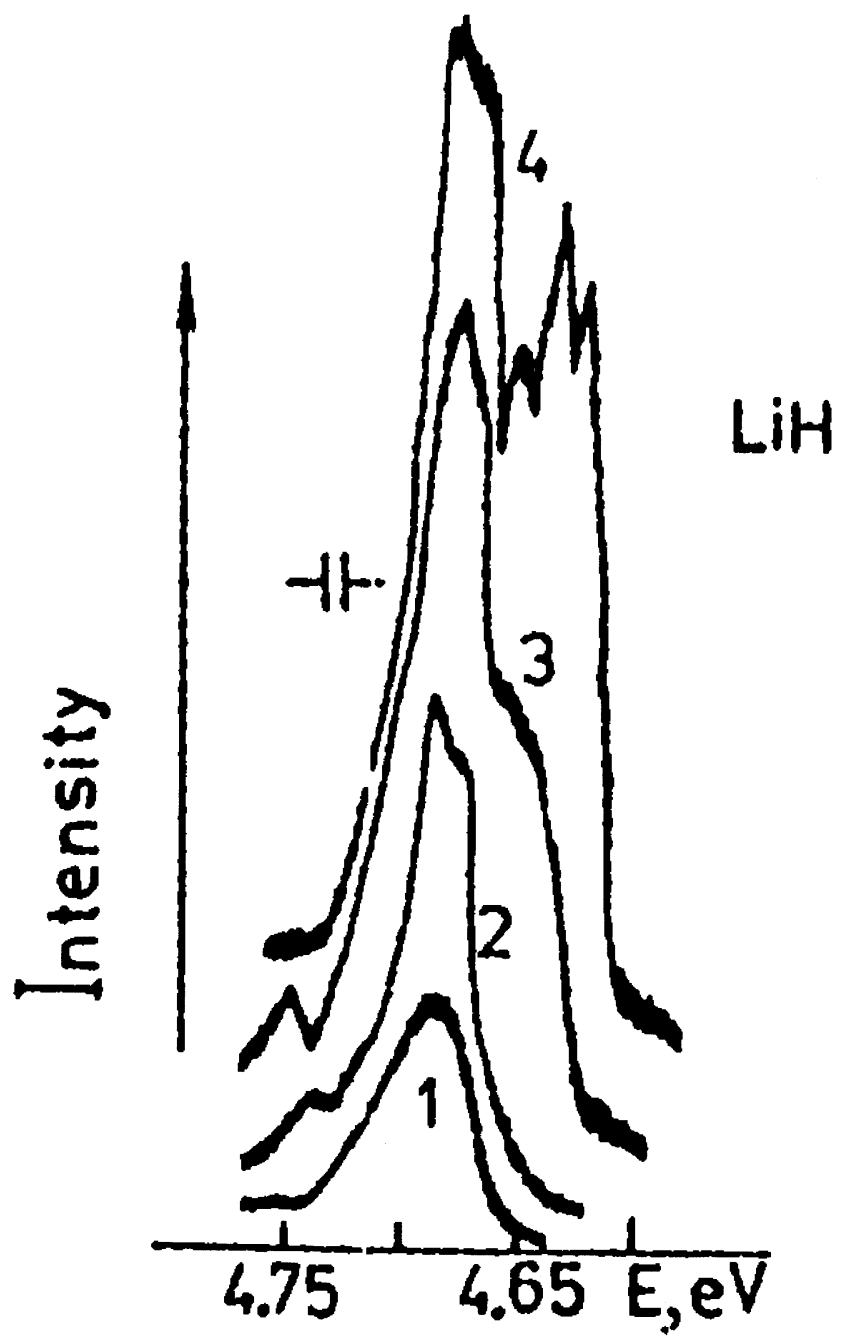

Figure 46. The dependence of the shape of $2 \mathrm{LO}$ replica line on the excitation intensity $\left(\mathrm{I}_{0}\right)$ light: $1-0.05 \mathrm{I}_{0} ; 2-0.09 \mathrm{I}_{0} ; 3-0.35 \mathrm{I}_{0} ; 4$ - $\mathrm{I}_{0}$ (After [272])

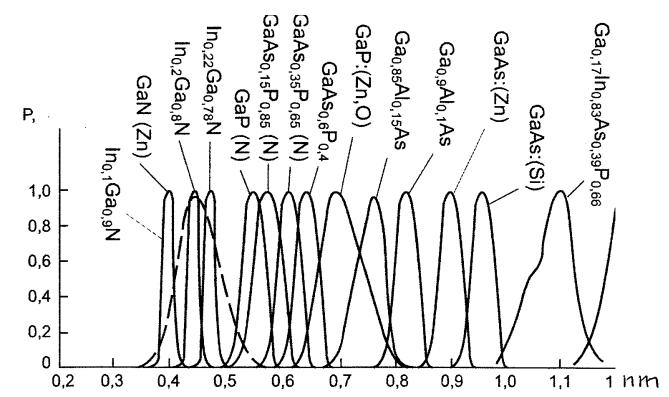

Figure 47. The spectra of light - emitting semiconductor diodes with different bandgaps (After [221]) 


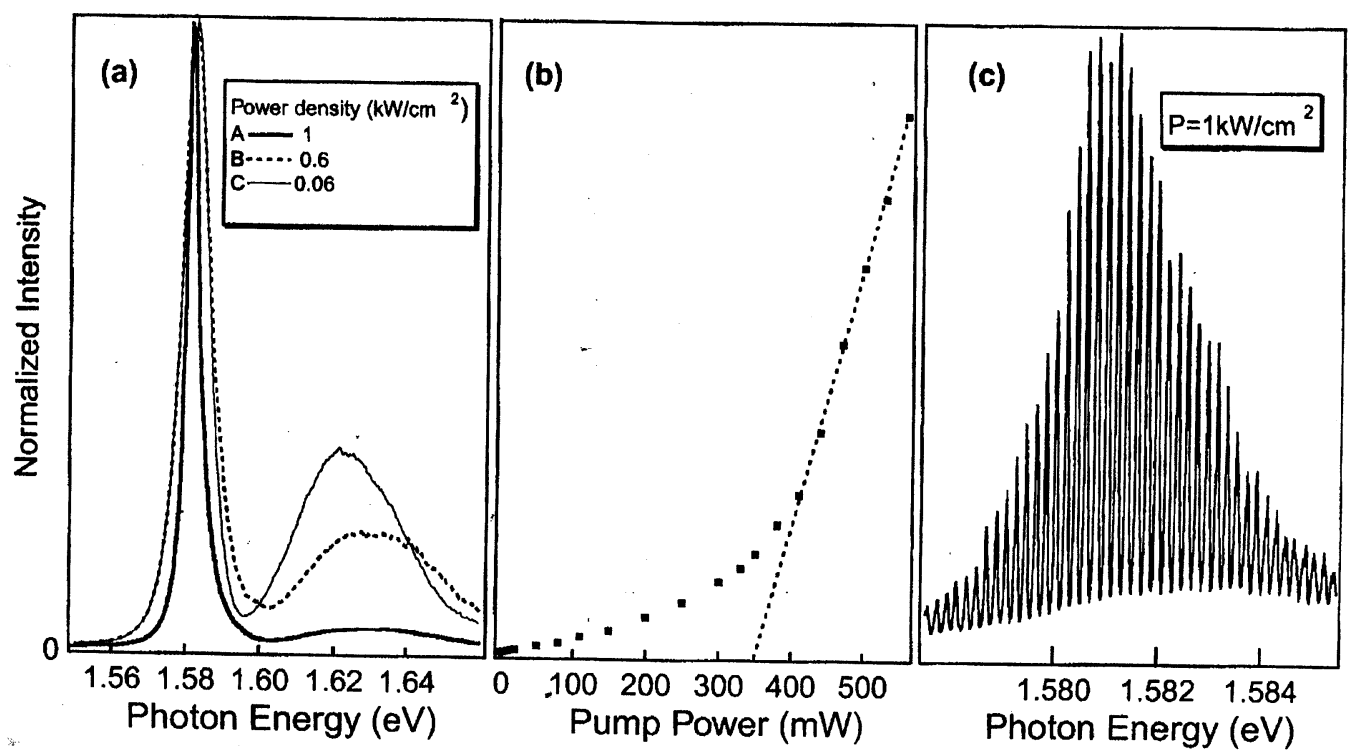

Figure 48. (a) Photoluminescence spectra at $10 \mathrm{~K}$ of the QWr laser sample above, below and near the lasing threshold in TE polarization. (b) Dependence on input excitation power of the PL output power;arrows indicate the excitation powers used for the optical spectra depicted in (a). (c) High resolution emission spectrum above the lasing threshold showing the Fabri - Perrot modes of the optical cavity (After [280])

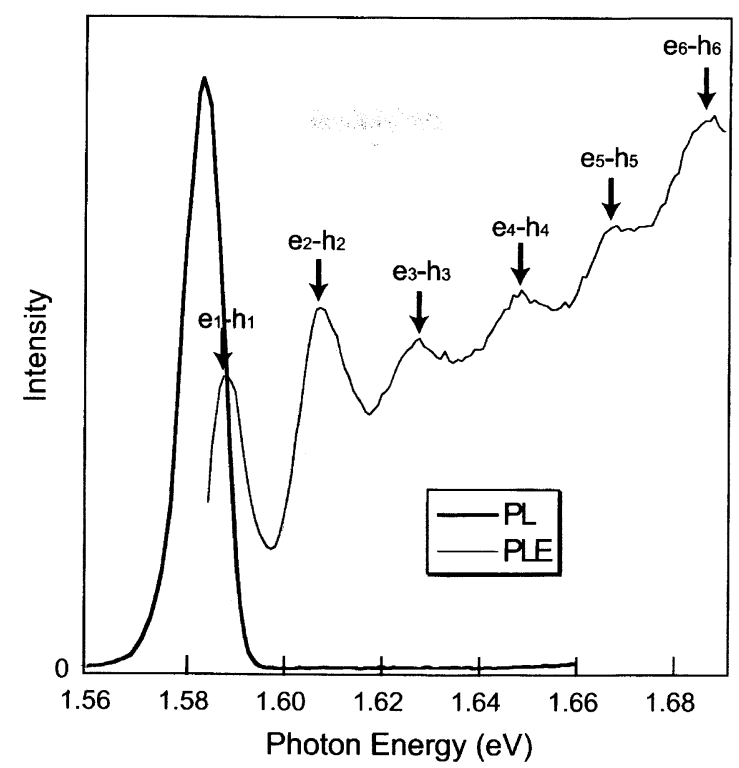

Figure 49. Linearly - polarized PLE spectrum and the corresponding PL spectrum of an etched QWr laser sample at $10 \mathrm{~K}$. The polarization of the excitation is parallel to the wire axis. The different optical transition $\mathrm{e}_{n}-\mathrm{h}_{n}$ are marked by arrows (After [280]) 
at $10 \mathrm{~K}$. The polarization of the excitation is parallel to the wire axis. The different optical transition $\mathrm{e}_{n}-\mathrm{h}_{n}$ are marked by arrows (After [260]).

In QDs, as indicated above, carriers are confined in the three directions in a very small region of space, producing quantum effects in the electronic properties. As we can see from [4], the electronic joint density of states for QD shows sharp peaks corresponding to transitions between discrete energy levels of electrons and holes. Outside these levels the DOS vanishes. In many ways, the electronic structure of a QD resembles that a single atom [259, 260]. Lasers based on QDs could have properties similar to those of conventional ion gas lasers, with the advantage that the electronic structure of a QD can be engineered by changing the base material, size and shape. In the next we assume that the QDs are small enough so that the separation between the first two electron energy levels for both electrons and holes is much larger than the thermal energy kT. Then for an undoped system, injected electrons and holes will occupy only the lowest level. Therefore, all injected electrons will contribute to the lasing transitions from the $\mathrm{E}_{1 e}$ to the $\mathrm{E}_{1 h h}$ levels, reducing the threshold current with respect to other systems with lower confinement. The evolution of the threshold current density obtained along the years for various laser structures is shown in Fig. 50. The lowest threshold currents have already been reached for QD lasers [262]. As long as the thermal energy is lower than the separation between the fist and second levels, the emission band in an ideal QD laser is very sharp and does not depend on temperature (see, also [17]. Therefore, QD lasers should have a better stability with temperature without the need for cooling. We should add that QD lasers have the narrowest spectrum and the highest gain (for details see, also [223, 224, 258]).

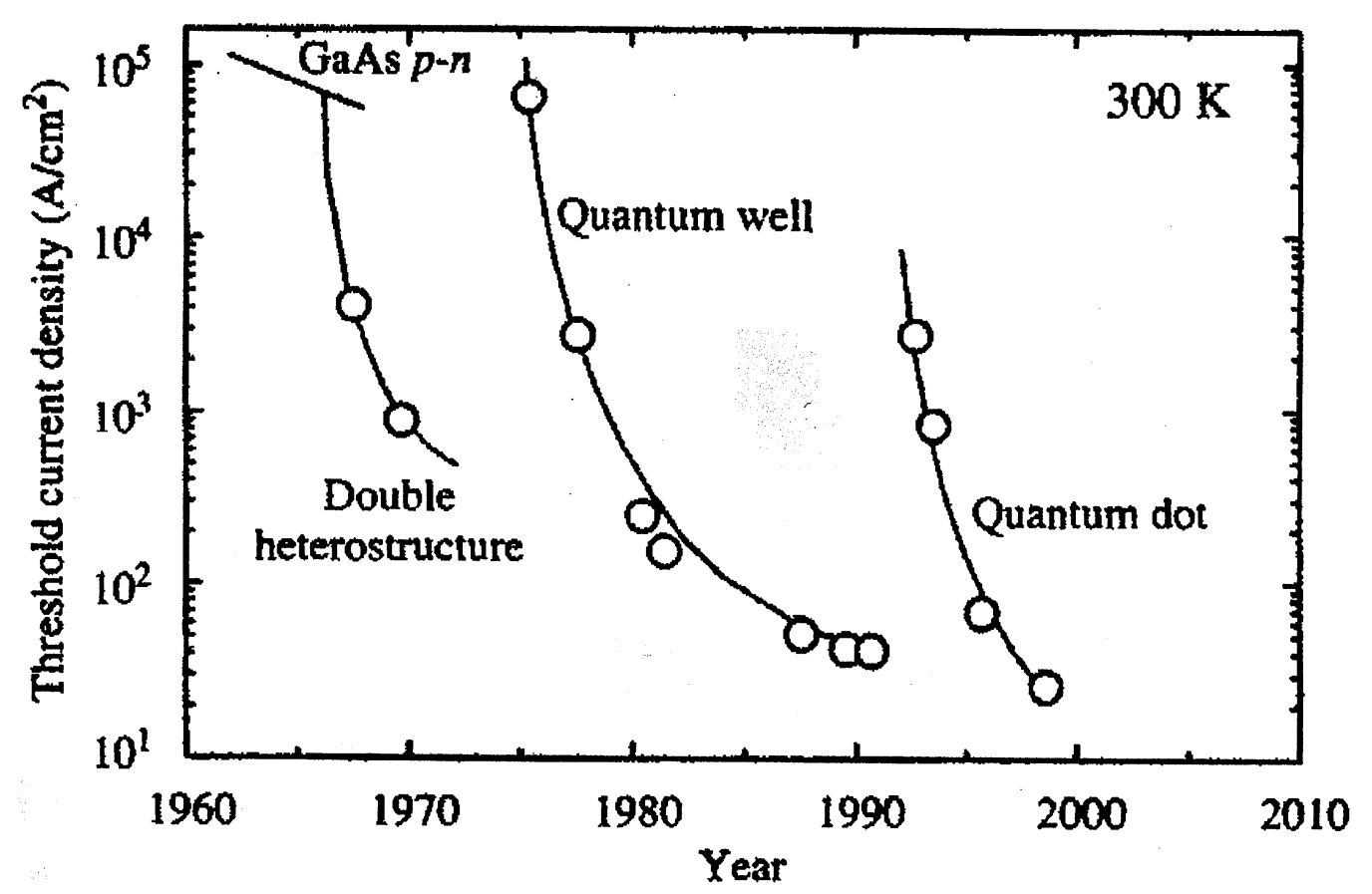

Figure 50. Evolution of threshold current density for lasers based on different confinement structures (After [4])

Fig. 50. Evolution of threshold current density for lasers based on different confinement structures (After [4]).

Quantum wells photodetectors. In principle, QWs can be used for the detection of light in any spectral region. As we all know the optical properties to interband transitions in QWs are quite different than those correspnding to bulk materials, since one has to consider both the $2 \mathrm{D}$ optical density of states [4] and the fact that excitonic absorption is much stronger in $2 \mathrm{D}$ systems. With respect to intraband transitions, in contrast to the 3D situation, 2D systems can show transitions without the necessity of involving phonons. Intraband transitions can be among electrons (or holes) in confined states in wells, or between confined states and the continuum (see, Fig. 51). These transition can be tailored for detection of light (IR photodetectors (see, e.g. [4, 17] and references therein). Thus, it is in the IR region between 2 and 20 microns, that QW photodetectors are preferably used for example in applications of night and thermal imaging (see, also $[4,17]$ ). The problem with photodiodes based on band - to band transitions across the band - gap $\mathrm{E}_{g}$ in $\mathrm{p}-\mathrm{n}$ homojunctions is that they require materials with very low values of $\mathrm{E}_{g}$, which makes it necessary to work at cryogenec temperatures. For instance, in the case of III - V compounds this leaves us with $\mathrm{InAs}_{1-x} \mathrm{Sb}_{x}$ with $\mathrm{x} \approx 0.5$. Some $\mathrm{A}_{2} \mathrm{~B}_{6}$ compounds like $\mathrm{HgCdTe}$ can also be used in the IR, but these materials are quite soft, difficult to process, and have large dark currents. Very perspective as QW potodetectors are isotope - mixed germanium as well as other isotope - mixed materials. In QWs, wavelength tunability is easily implemented since the energies of the levels in a QW can be adjusted by the fabrication parameters, in particular its width. Fig. 51 show the absorption transitions suitable for IR detection for a single QW under the action of an applied electric field, although practical devices are made with MQWs. In Fig. $51^{a}$ there are two energy levels in each well, the second level being located close to the top of the barriers. The separation between levels should be in the range $0.1-0.2 \mathrm{eV}$, which for III - V compounds implies a width of 
the wells about $10 \mathrm{~nm}$ [230]. Sometimes, it is more effective to make use of absorption transitions between a single level in the well and the first continuum narrow band outside it (Fig. $51^{b}$ ). In the case of the system AlGaAs - GaAs -AlGaAs, this energy is about $0.12 \mathrm{eV}$, and therefore, the spectral response is around 10 $\mu \mathrm{m}$ [256]. The advantage of using this scheme is that the photodetector dark current is smaller than for the previous case in which the carriers had to leave the wlls by tunneling (for details, see [259]).

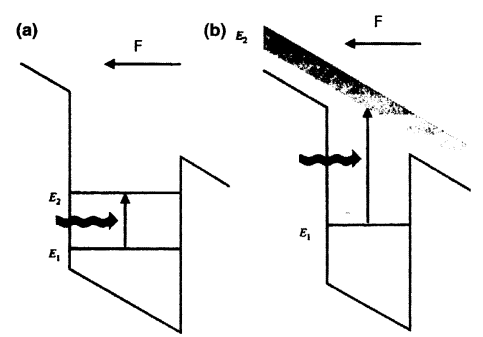

Figure 51. Optical absorption transition for IR detection in a QW: a) intersubband transitions; b) transion from a bound state to the continuum narrow band outside the potential wells. $\mathrm{F}$ is the applied electric field

Fig. 51. Optical absorption transition for IR detection in a QW: a) intersubband transitions; b) transion from a bound state to the continuum narrow band outside the potential wells. $\mathrm{F}$ is the applied electric field.

\section{Conclusion}

In this review, we have presented briefly the results of experimental and theoretical studies of the objects of research that earlier were simply in accessible (naturally with exception of $\mathrm{LiH}_{x} \mathrm{D}_{1-x}$ crystals). This new branch of material science which is called the isotope - based material science. The use of such objects allows the investigation of not only the isotope effects in lattice dynamics (elastic, thermal and vibrational properties) but also the influence of such effects on the electronic states via electron-phonon coupling (the renormalization of the band-to-band transition energy $\mathrm{E}_{g}$, the exciton binding energy $\mathrm{E}_{B}$ and the size of the longitudinal-transverse splitting $\left.\Delta_{L T}\right)$.

Substituting a light isotope with a heavy one increases the interband transition energy $\mathrm{E}_{g}$ (excluding $\mathrm{Cu}$-salts) and the binding energy of the Wannier-Mott exciton $\mathrm{E}_{B}$ as well as the magnitude of the longitudinal-transverse splitting $\Delta_{L T}$. The nonlinear variation of these quantites with the isotope concentration is due to the isotopic disordering of the crystal lattice and is consistent with the concentration dependence of line halfwidth in exciton reflection and luminescence spectra. A comparative study of the temperature and isotopic shift of the edge of fundamental absorption for a large number of different semiconducting and insulating crystals indicates that the main (but not the only) contribution to this shift comes from zero oscillations whose magnitude may be quite considerable and comparable with the energy of LO phonons. The theoretical description of the experimentally observed dependence of the binding energy of the Wannier-Mott exciton $\mathrm{E}_{B}$ on the nuclear mass requires the simultaneous consideration of the exchange of LO phonons between the electron and hole in the exciton, and the separate interactions of carriers with LO phonons. The experimental dependence $\mathrm{E}_{B} \sim \mathrm{f}(\mathrm{x})$ for $\mathrm{LiH}_{x} \mathrm{D}_{1-x}$ crystals fits in well enough with the calculation according to the model of large-radius exciton in a disordered medium; hence it follows that the fluctuation smearing of the band edges is caused by isotopic disordering of the crystal lattice.

The wide possibilities of isotope - based materials science discussed in this review hold the greatest promise for application in solid-state and quantum electronics, biology, human memory, optoelectronics, different electronic devices, electronic and quantum computers, and many other modern and new technologies that are even now difficult to imaginate. And we should repeat that the main aim of this review is to familiarize readers with present and some future applications in isotope- based materials science.

\section{Acknowledgements}

I would like to express my deep thanks to many authors and publishers whose Figures and Tables I used in my review. In a few cases I have been unable to contact the authors, and I would be grateful if they would nevertheless retrospectively give me the necessary permission. I would like to express thanks to my many students who have contributed to this investigations in various ways over the years. Many thanks are due to Prof. W. Beder for carefully reading of my manuscript as well as Dr. P. Night for improving my English. 


\section{Tables}

Table 1. Calculated energy values of some direct optical transitions in $\mathrm{LiH}$ reduced to the experimental value of $\mathrm{E}_{g}=5.0 \mathrm{eV}$.

$\begin{array}{lllll}\text { Transition } & 1 & 2 & 3,4 & 5 \\ \mathrm{~K}_{1}-\mathrm{K}_{3} & 6.9 & 7.5 & 6.5 & 6.4 \\ \mathrm{~W}_{1}-\mathrm{W}_{3} & 8.0 & 7.9 & 7.3 & 7.4 \\ \mathrm{~L}_{1}-\mathrm{L}_{2}^{\prime} & 9.2 & 9.6 & 9.0 & 9.1 \\ \mathrm{~W}_{1}-\mathrm{W}_{2} & 12.6 & 14.9 & 12.2 & \\ \mathrm{X}_{1}-\mathrm{X}_{5}^{\prime} & 12.9 & 13.8 & 13.6 & \\ \mathrm{~K}_{1}-\mathrm{K}_{4} & 14.7 & 16.1 & 15.0 & \\ \mathrm{~L}_{1}-\mathrm{L}_{3} & 19.7 & 20.9 & 20.7 & \\ \Gamma_{1}-\Gamma_{15} & 24.5 & 25.3 & 33.3 & \end{array}$

Table 2. The energy shifts of all of the transitions studied in $\left[93^{a}\right]$ are given in terms of the $\mathrm{Cd}^{34} \mathrm{~S}$ minus the $\mathrm{Cd}^{\text {nat }} \mathrm{S}$ energy, $\Delta \mathrm{E}$.

$\begin{array}{lll}\text { Transition } & \text { Method } & \Delta \mathrm{E}\left(\mathrm{cm}^{-1}\right) \\ \mathrm{I}_{2} & \mathrm{PL} & 10.6 \pm 0.1 \\ \mathrm{I}_{2}^{z} & \mathrm{PL} & 11.1 \pm 0.1 \\ \mathrm{I}_{2}^{a} & \mathrm{PL} & 10.6 \pm 0.1 \\ \mathrm{~A}_{n=1}\left(\Gamma_{6}\right) & \mathrm{A} \| & 10.8 \pm 0.2 \\ \mathrm{~A}_{n=1}\left(\Gamma_{5}^{L}\right) & \mathrm{PL} & 11.0 \pm 0.2 \\ \mathrm{~A}_{n=1}\left(\Gamma_{5}^{L}\right) & \mathrm{R} \perp & 10.9 \pm 0.2 \\ \mathrm{~A}_{n=2} & \mathrm{PL} \| & 11.3 \pm 0.4 \\ \mathrm{~A}_{n=2} & \mathrm{PL} \perp & 11.1 \pm 0.4 \\ \mathrm{~A}_{n=2} & \mathrm{R} \perp & 10.2 \pm 0.5 \\ \mathrm{~A}_{n=3} & \mathrm{PL} \| & 11.8 \pm 1.1 \\ \mathrm{~A}_{n=3} & \mathrm{PL} \perp & 10.9 \pm 0.6 \\ \mathrm{~A}_{n=3} & \mathrm{R} \perp & 10.7 \pm 0.6 \\ \mathrm{~B}_{n=1}\left(\Gamma_{1}\right) & \mathrm{R} \| & 10.9 \pm 0.3 \\ \mathrm{~B}_{n=1}\left(\Gamma_{5}^{L}+\Gamma_{5}^{T}\right) & \mathrm{R} \perp & 10.6 \pm 0.4 \\ \mathrm{~B}_{n=2} & \mathrm{R} \| & 9.4 \pm 1.2 \\ \mathrm{~B}_{n=2} & \mathrm{R} \perp & 9.8 \pm 1.2 \\ \mathrm{C}_{n=1}\left(\Gamma_{1}\right) & \mathrm{R} \| & 15 \pm 6 \\ \mathrm{C}_{n=1}\left(\Gamma_{5}\right) & \mathrm{R} \perp & 14 \pm 5\end{array}$

The methods used were photoluminescence spectroscopy (*PL) and reflection spectroscopy (R). For measurements made using polarized light, the $\|$ or $\perp$ specifies the orientation of the $\mathbf{E}$ vector vs the $\mathrm{c}$ axis.

Table 3. The change of the value of the exciton binding energy $\left(\Delta \mathrm{E}_{B}\right)$, band-to-band transitions $\left(\Delta E_{g}\right)$ at the $100 \%$ isotopical substitution. All values indicate in $\mathrm{meV}_{\text {or }} \mathrm{cm}^{-1}$. (After Plekhanov, 1998)

\begin{tabular}{|c|c|c|}
\hline Compound & $\Delta E_{g}$ & $\Delta E_{B}$ \\
\hline $\mathrm{Ge}^{70} \rightarrow G e^{76}$ & indirect (L-point) $2.1 \mathrm{mev}=16.94 \mathrm{~cm}^{-1}[1,2,3]$ & - \\
\hline $\operatorname{GaAs}\left(\mathrm{Ga}^{69} \rightarrow G a^{71}\right)$ & $\operatorname{direct}(\Gamma-$ point $) 0.85 \mathrm{mev}=10.5 \mathrm{~cm}^{-1}[4]$ & - \\
\hline $\mathrm{Cu}_{2} \mathrm{O}\left(\mathrm{O}^{16} \rightarrow O^{18}\right)$ & direct (yel) $2.2 \mathrm{mev}=18 \mathrm{~cm}^{-1}[5]$ & - \\
\hline $\mathrm{Cu}_{2} \mathrm{O}\left(\mathrm{O}^{16} \rightarrow \mathrm{O}^{18}\right)$ & direct (green) $2.2 \mathrm{mev}=18 \mathrm{~cm}^{-1}[5]$ & - \\
\hline $\operatorname{CdS}\left(\mathrm{S}^{32} \rightarrow \mathrm{S}^{34}\right)$ & - & $-1.6 \mathrm{meV}[7]$ \\
\hline $\mathrm{CdS}\left(\mathrm{Cd}^{112} \rightarrow C d^{\text {nat }}\right)$ & direct $0.003 \mathrm{meV}$ & $0.4 \mathrm{meV}[8]$ \\
\hline $\mathrm{CuCl}\left(\mathrm{Cu}^{63} \rightarrow \mathrm{Cu}^{65}\right)$ & $(\Gamma-$ point $)-0.155 \mathrm{meV}=-1.24 \mathrm{~cm}^{-1}[9]$ & - \\
\hline $\mathrm{ZnO}\left(\mathrm{O}^{16} \rightarrow \mathrm{O}^{18}\right)$ & $(\Gamma-$ point $) 6.82 \mathrm{meV}=55 \mathrm{~cm}^{-1}[10,11]$ & - \\
\hline $\mathrm{ZnO}\left(\mathrm{Zn}^{64} \rightarrow Z n^{68}\right)$ & $(\Gamma-$ point $) 1.5 \mathrm{meV}=12 \mathrm{~cm}^{-1}[10,11]$ & - \\
\hline $\mathrm{C}^{12} \rightarrow C^{13}$ & indirect $(\Gamma-$ point $) 13.6 \mathrm{meV}=109.7 \mathrm{~cm}^{-1}[12]$ & - \\
\hline $\mathrm{LiH}(\mathrm{H} \rightarrow D)$ & direct $103 \mathrm{meV}=831 \mathrm{~cm}^{-1}[15,16,17]$ & $10 \mathrm{meV}[16]$ \\
\hline $\mathrm{LiH}\left(\mathrm{Li}^{6} \rightarrow L i^{7}\right)$ & direct $5 \mathrm{meV}=41 \mathrm{~cm}^{-1}[16]$ & $3 \mathrm{meV}[16]$ \\
\hline
\end{tabular}

\section{REFERENCES}

1. V.F. Agekyan et al, Fizika Tverdogo Tela, 31, 101 (1989) (in Russian) .

2. C. Parks et al, Phys. Rev., B49, 14244 (1994).

3. J.J.Haller, J. Appl. Phys. 77, 2857 (1995).

4. N. Garro et al, Phys. Rev., B54, 4732 (1996).

5. F.I. Kreingol'd et al, Pis'ma v ZETPH, 23, 679 (1976) (in Russian).

6. F.I. Kreingol'd, Fizika Tverdogo Tela, 27, 2839 (1985) (in Russian).

7. F.I. Kreingol'd et al, ibid, 26, 3490 (1984) (in Russian).

8. J.M. Zhang et al, Phys. Rev. B57, 9716 (1998)

9. N. Garro et al, Solid State Commun., 98, 27 (1996).

10. F.I. Kreingol'd, Fizika Tverdogo Tela 20, 3138 (1978) (in Russian). 
11. F.I. Kreingol'd and B.S. Kulinkin, ibid, 28, 3164 (1986) (in Russian).

12. T. Collins et al, Phys. Rev. Lett., 65, 891 (1990).

13. R.M. Chrenko, J. Appl. Phys. 63, 5873 (1988).

14. K.S. Hass et al, Phys. Rev. B54, 7171 (1992).

15. A.A. Klochikhin and V.G.Plekhanov, Fizika Tverdogo Tela, 22,585 (1980) (in Russian).

16. V.G. Plekhanov, Uspekhi Fiz. Nauk, 167, 577 (1997); Plekhanov V.G and Plekhanov N.V. Phys. Lett. A313, 231(2003).

17.A.F. Kapustinsky et al, Physicochimica USSR, 7, 799 (1937).

Table 4. Values of the energy of maxima (in meV) in exciton reflection spectra of pure and mixed crystals at $2 \mathrm{~K}$, and energies of exciton binding $\mathrm{E}_{\mathrm{b}}$, band-to-band transitions $\mathrm{E}_{\mathrm{g}}$ (After [1]).

\begin{tabular}{|c|c|c|c|c|c|}
\hline Energy, meV & $\mathrm{LiH}$ & $\mathrm{LiH}_{0.82} \mathrm{D}_{0.18}$ & $\mathrm{LiH}_{0.40} \mathrm{D}_{0.60}$ & $\mathrm{LiD}$ & ${ }^{6} \mathrm{LiH}(78 \mathrm{~K})$ \\
\hline $\mathrm{E}_{1 \mathrm{~s}}$ & 4950 & 4967 & 5003 & 5043 & 4939 \\
\hline $\mathrm{E}_{2 \mathrm{~s}}$ & 4982 & 5001 & 5039 & 5082 & 4970 \\
\hline $\mathrm{E}_{\mathrm{b}}$ & 42 & 45 & 48 & 52 & 41 \\
\hline $\mathrm{E}_{\mathrm{g}}$ & 4992 & 5012 & 5051 & 5095 & 4980 \\
\hline
\end{tabular}

Table 5. Values of the Coefficients $\partial \mathrm{E}_{\mathrm{g}} / \partial \mathrm{M}(\mathrm{meV})$ and Energies of the Band-to-Band Transitions $\mathrm{E}_{\mathrm{g}}(\mathrm{eV})$ according to indicated references (after [4]).

$\begin{array}{lll}\text { Substance } & \partial \mathrm{E}_{\mathrm{g}} / \partial \mathrm{M}(\mathrm{meV}) & \mathrm{E}_{\mathrm{g}}(\mathrm{eV}) \\ { }^{13} \mathrm{C} \longrightarrow{ }^{12} \mathrm{C} & 14.6[1] & 5.4125[1] \\ { }^{7} \mathrm{LiH} \longrightarrow{ }^{7} \mathrm{LiD} & 103[2] & 4.992 \longrightarrow 5.095[2] \\ { }^{7} \mathrm{LiH} \longrightarrow{ }^{6} \mathrm{LiH} & 12[2] & 4.980[2] \\ \mathrm{CsH} \longrightarrow \mathrm{CsD} & 60[3] & 4.440[3] \\ { }^{30} \mathrm{Si} \longrightarrow{ }^{28} \mathrm{Si} & 2[4] & 3.652[4] \\ { }^{30} \mathrm{Si} \longrightarrow{ }^{28} \mathrm{Si} & 2.09[5] & 1.166[5] \\ { }^{68} \mathrm{ZnO} \rightarrow{ }^{64} \mathrm{ZnO} & 0.372[6] & 3.400[6] \\ \mathrm{Zn}^{18} \mathrm{O} \rightarrow \mathrm{Zn}^{16} \mathrm{O} & 3.533[6] & 3.400[6] \\ { }^{68} \mathrm{ZnO} \rightarrow{ }^{64} \mathrm{ZnO} & 0.40[7] & 3.400[6] \\ \mathrm{Zn}^{18} \mathrm{O} \rightarrow \mathrm{Zn}^{16} \mathrm{O} & 3.20[7] & 3.400[6] \\ { }^{69} \mathrm{GaP} \longrightarrow{ }^{71} \mathrm{GaP} & 0.19[8] & 2.400[8] \\ { }^{65} \mathrm{CuCl} \rightarrow{ }^{63} \mathrm{CuCl} & -0.076[9] & 3.220[9] \\ \mathrm{Cu}^{37} \mathrm{Cl} \rightarrow \mathrm{Cu}{ }^{35} \mathrm{Cl} & 0.364[10] & 3.220[10] \\ \mathrm{Cd}^{34} \mathrm{~S} \rightarrow \mathrm{Cd}^{32} \mathrm{~S} & 0.370[11] & 2.580[11] \\ { }^{110} \mathrm{CdS} \rightarrow{ }^{116} \mathrm{CdS} & 0.040 \div 0.068[12] & 2.580[11] \\ \mathrm{Cu}_{2}^{18} \mathrm{O} \rightarrow \mathrm{Cu}{ }_{2}^{16} \mathrm{O} & 1.116[13] & 2.151[13] \\ { }^{71} \mathrm{GaAs} \rightarrow{ }^{69} \mathrm{GaAs} & 0.39[9] & 1.53[9] \\ { }^{76} \mathrm{Ge} \rightarrow 72 \mathrm{Ge} & 0.225[11-16] & 1.53[11-16] \\ { }^{76} \rightarrow{ }^{73} \rightarrow{ }^{70} \mathrm{Ge} & 0.37[15-16] & 0.74[15-16] \\ { }^{7} \mathrm{Col} & \end{array}$

1. T. Collins et al., Phys. Rev. Lett. 65, 891 (1990).

2. V.G. Plekhanov, Uspekhi Fiz. Nauk (Moscow) 167, 577 (1997) (in Russian).

3. K. Chandahari et al. Solid State Commun. 100, 777 (1996) .

4. L.F. Lastras - Martinez et al. Phys. Rev. B61, 12946 (2000) .

5. D. Karaiskaja et al. Solid State Commun. 123, 87 (2002).

6. F.I. Kreingol'd, Fizika Tverdogo Tela 20, 3138 (1978)(in Russian).

7. F.J. Manjon et al. Solid State Commun. 128, 35 (2003).

8. T.A. Meyer et al. Solid State Commun. 126, 119 (2003) .

9. N. Garro et al. Phys. Rev. B54, 4732 (1996) .

10. N. Garro et al. Solid State Commun. 98, 27 (1996).

11. F.I. Kreingol'd and B.S. Kulinkin, Fizika Tverdogo Tela 28, 3164 (1986) (in Russian).

12.T.A. Meyer et al., Phys. Rev. B69, 115214 (2004).

13. F.I. Kreingol'd, Pis'ma v ZETPH 23 (1976) 679; F.I. Kreingol'd, Fizika Tverdogo Tela 27, 2839 (1985) (in Russian).

14. V.F. Agekyan et al. Fizika Tverdogo Tela 31, 101 (1989) (in Russian).

15. C. Parks et al. Phys. Rev. B49, 14244 (1994) .

16. E.E. Haller, J. Appl. Phys. 77, 2857 (1995) . 
Table 6. $\alpha, \mathrm{s} \cdot 10^{6}$ for isotopic LiH (After [181])

$\begin{array}{llll}\text { Isotopic composition } & -190-25^{0} \mathrm{C} & 25-140^{0} \mathrm{C} & 140-240^{0} \mathrm{C} \\ { }^{6} \mathrm{LiH} & 21.0 \pm 0.3 & 34.4 \pm 0.8 & 50.0 \pm 1.0 \\ { }^{7} \mathrm{LiH} & 19.8 \pm 0.4 & 37.4 \pm 0.4 & 53.3 \pm 0.6 \\ { }^{6} \mathrm{LiD} & 24.0 \pm 1.0 & 38.4 \pm 1.8 & 54.3 \pm 1.0 \\ { }^{7} \mathrm{LiD} & 24.8 \pm 0.4 & 42.9 \pm 1.0 & 55.0 \pm 1.4 \\ { }^{7} \mathrm{LiT} & 26.4 \pm-.5 & & \end{array}$

Table7. Ratio of $\alpha, \mathrm{s}$ for isotopic LiH (After [181]).

$\begin{array}{llll}\text { Ratio } & -190-25{ }^{0} \mathrm{C} & 25-140^{0} \mathrm{C} & 140-240{ }^{0} \mathrm{C} \\ \frac{\alpha_{\mathrm{H}}}{\alpha_{\mathrm{D}}} \text { for }{ }^{6} \mathrm{Li} & 0.879 & 0.896 & 0.921 \\ \frac{\alpha_{\mathrm{H}}}{\alpha_{\mathrm{D}}} \text { for }{ }^{7} \mathrm{Li} & 0.805 & 0.872 & 0.969 \\ \frac{\alpha\left({ }^{6} \mathrm{Li}\right)}{\alpha\left({ }^{7} \mathrm{Li}\right)} \text { for } \mathrm{H} & 1.060 & 0.920 & 0.982 \\ \frac{\alpha_{\mathrm{H}}}{\alpha_{\mathrm{T}}} \text { for }{ }^{7} \mathrm{Li} & 0.750 & & \\ \frac{\alpha_{\mathrm{D}}}{\alpha_{T}} \text { for }{ }^{7} \mathrm{Li} & 0.932 & & \end{array}$

Table 8. Comparison of the effect of isotopic scattering on the thermal conductivity of natural Ge, Si and diamond at $300 \mathrm{~K}$. The $\%$ increase in $\mathrm{k}$ is the increase of the thermal conductivity of the nearly isotopically pure sample compared to the natural sample. The isotope is defined as $\mathrm{g}=\sum_{i} \mathrm{f}_{i}\left(\frac{\Delta \mathrm{M}_{i}}{\mathrm{M}}\right)^{2}($ After [211], [213] and [214]).

$\begin{array}{llll} & \% \text { increase in } \mathrm{k} & \theta & \Gamma \cdot 10^{-4} \\ \mathrm{Ge} & 30 & 376 & 5.80 \\ \mathrm{Si} & 60 & 658 & 2.01 \\ \text { Diamond } & 50 & 1860 & 0.76\end{array}$

\section{REFERENCES}

1. V.G. Plekhanov, Elementary excitations in isotope - mixed crystals, Phys. Reports 410, 1 - 235 (2005).

2. M. Cardona and M.L.W. Thewalt, Isotope effects on the optical spectra semiconductors, Rev. Mod. Phys. 77, 1173 - 1224 (2005).

3. K. Goser, Glösekötter, J. Dienstuhl, Nanoelectronics and Nanosystems, (Springer, Berlin, 2004).

4. V.G. Plekhanov, Isotope Low - Dimensional Structures ( (Springer, Berlin, 2012).

5. F. Soddy, Inta - atomic charge, Nature (London) 92, 399 - 400 (1913).

6. F. Soddy, The structure of the atom, Nature (London) 92, 452 - 452 (1913)

7. J.J. Thomson, On the appearance of helium and neon in vacuum tubes, Nature (London) 90, 645 - 647 (1913).

$7^{a}$. H.C. Urey and G.K. Teal, The hydrogen isotope of atomic weight two, Rev. Mod. Phys. 7, 34 - 94 (1935).

8. C.M. Lederer, V.S. Shirley, Table of Isotopes (Wiley, New York, 1978).

9. J. Emsley, The Elements (Oxford University Press, Oxford, 1990).

10. http:// physics.nist.gov/PhysRefData/Compositions/index.html.

11. E.M. Burbidge, G.R. Burbidge, W.A. Fowler, F. Hoyle, Synthesis of the elements in stars, Rev. Mod. Phys. 29, 547 - 652 (1957).

12. G. Wallerstein, I. Jhen, Jr, P. Parker et al., Synthesis of the elements in stars: forty years in progress, ibid, 69, 995 - 1084 (1997).

13. S. Esposito, Primordial nucleosynthesis: accurate prediction for light elements abundances, ArXiv: astro phys / 990441.

14. See, e.g. E.N. Ramsden, A - Level Chemistry (Hull, Stanley, Thornes Publishers, 1985).

15. L.J. Malone, Basic Conxepts of Chemistry (Wiley, New York, 2003).

16. V.G. Plekhanov, Manifestation and origin of the isotope effect, ArXiv:gen.phys/0907.2024.

17. V.G. Plekhanov, Isotopes in Condensed Matter (Springer, Berlin - Heidelberg, 2012).

18. S. Villani, Isotope Separation (American Nuclear Society, Hinsdale, IL, 1976).

19. E.E. Haller, Isotopically engineered semiconductor, J. Appl. Phys. 77, 2857 - 2877 (1995).

20. V.G. Plekhanov, Isotope effect in lattice dynamics, Physics - Uspekhi 46, 689 - 715 (2003).

21. V.G. Plekhanov, Experimental evidence of strong scattering in isotopical disordered systems: the case $\mathrm{LiH}_{x} \mathrm{D}_{1-x}$, Phys. Rev. B51, 8874 - 8878 (1995).

22. V.G. Plekhanov, Giant Isotope Effect in Solids (Stefan University Press, La Jolla, 2004).

23. G. Leibfried and W. Ludwig, Theory of Anharmonic Effect in Crystals (Academic Press, New York, 1961). 
24. A. Barker and A.J. Sievers, Optical studies of the vibrational properties of disordered solids, Rev. Mod. Phys. 47, Suppl. 2, S1 - S179 (1975).

25. N.F. Mott, R.V. Gurney, Electron Processes in Ionic Crystals (Clarendon Press, Oxford, 1948).

26. J. Slater, The Self - Consistent Field for Molecules and Solids (McGraw - Hill, New York, 1975).

27. W.B. Fowler, Influence of electronic polarization on the optical properties of insulators, Phys. Rev. 151, $657-667(1966)$.

28. D.H. Ewing, F. Seitz, On the electronic constitution of crystals: LiF and LiH, Phys. Rev. 50, 760 - 777 (1936).

29. F. Perrot, Bulk properties of lithium hydride up to 100 Mbar, Phys. Stat. Sol. (b) 77, 517 - 525 (1976).

30. G.S. Zavt, K. Kalder et al., Electron excitation and luminescence LiH single crystals, Fiz. Tverd. Tela (St. Petersburg) 18, 2724 - 2730 (1976) (in Russian).

31. N.I. Kulikov, Electron structure, state equation and phase transitions insulator - metal in hydride lithium, ibid, 20, 2027 - 2035 (1978) (in Russian).

32. G. Grosso, G.P. Parravichini, Hartree - Fock energy bands by the orthogonalized - plane - wave method: lithium hydride results, Phys Rev. B20, 2366 - 2372 (1979).

33. S. Baroni, G.P.Parravichini, G. Pezzica, Quasiparticle band structure of lithium hydride, Phys. Rev. B32, 4077 - 4087 (1985).

34. A.B. Kunz, D.J. Mikish, Electronic structure of LiH and NaH, ibid, B11, 1700 - 1704 (1975).

35. J. Hama, N. Kawakami, Pressure induced insulator - metal transition in solid LiiH, Phys. Lett. A126, 348 - 352 (1988).

36. T.A. Betenekova, I.N. Shabanova, F.F. Gavrilov, The structure of valence band in lithium hydride, Fiz. Tverd. Tela 20, 2470 - 2477 (1978) (in Russian).

37. K. Ichikawa, N. Susuki, K. Tsutsumi, Photoelectron spectroscopic study of LiH, J.Phys. Soc. Japan 50, 3650 - 3654 (1981).

38. R.A. Kink, M.F. Kink, T.A. Soovik, Reflection spectra of lithium hydride crystals in $4-25 \mathrm{eV}$ at $5 \mathrm{~K}$, Nuclear Instruments and Methods in Physics Research, A261, 138 - 140 (1987).

39. V.G. Plekhanov, Isotopic and disorder effects in a large exciton spectroscopy, Physics - Uspekhi 40, 553 579 (1997).

40. V.G. Plekhanov, V.I. Altukhov, Determination of exciton and exciton - phonon interaction parameters via resonant secondary emission of insulators, Sov. Phys. Solid State 23, 439 - 447 (1981).

41. V.G. Plekhanov, Nuclear technology of the creation of quantum dots in graphene, Sci. Transaction of SHI, Tallinn, 2011, p.p. 66 -71 (in Russian).

$41^{a}$. V.G. Plekhanov, Manifestation of the strong nuclear interaction in the isotope - induced band - gap opening of graphene, in, Horizons in World Physics, Vol. 281 (Nova Science Publishers, Inc., New York, 2013), p.p. $197-202$.

42. A.A. Blistanov, Acoustic Crystals: Handbook, M.T. Shchaskol'skaya (ed.) ( Nauka, Moscow,1982) (in Russian).

43. R.M. Martin, Electronic Structure - Basic Theory and Practical Methods (Cambridge University Press, Cambridge, 2004).

44. A.S. Davydov, Theory of Solids (Science, Moscow, 1976) (in Russian).

45. C. Kittel, Introduction to Solid State Physics ( Wiley, New York,1986).

46. N.W. Ashcroft, N.D. Mermin,Solid State Physics (Holt, Reinhart and Winston, New York, 1976).

47. R. Wyckoff, Crystal Structures (Interscience, New York, 1963).

48. M.L. Cohen, J. Chelikowsky, Electronic Properties and Optical Properties of Semiconductors, 2nd. ed., Springer Series Solid - State Sci., Vol. 75 (Springer, Berlin - Heidelberg, 1989).

49. J.I. Pankove, Optical Processes in Semiconductors (Prentice - Hall, Inc., Englewood Cliffs, New Jersey, 1971).

50. J.I. Frenkel, On the transformation light into heat in solids, I, Phys. Rev.37: 17 - 44 (1931); On the transformation light into heat in solids, II, 37, 1276 - 1294 (1931).

51. R. Peierls, Ann. Phys. 31, 905 (1932).

52. J.K. Slater and W.H. Shockley, Optical absorption by alkali halides, Phys. Rev. 50, 705 - 719 (1936) .

53. G.H. Wannier, Structure of electronic excitation levels in insulating crysals, Phys. Rev. 52, 191 - 197 (1937).

54. N. F. Mott and M. J. Littleton, Conduction in polar crystals. I. Electrolytic conduction in solid salts, Trans. Faraday Soc. 34, 485 - 499 (1938); N.F. Mott, Conduction in polar crystals. II. The conduction band and ultra-violet absorption of alkali-halide crystals, Trans. Faraday Soc. 34, 500 - 505 (1938);R. W. Gurney and N. F. Mott, Conduction in polar crystals. III. On the colour centres in alkali-halide crystals, Trans. Faraday Soc. 34, $506-511$ (1938).

55. A.S. Davydov, Theory of Molecular Excitons (Science, Moscow, 1968) (in Russian).

56. V.M. Agranovich, Theory of Excitons (Science, Moscow, 1976) (in Russian).

57. V.M. Agranovich and V.L. Ginzburg, Crystallooptic and the Theory of Excitons (Science, Moscow, 1979)

(in Russian).

58. E.F. Gross, Selected Papers ( Science, Leningrad,1976) (in Russian). 
59. V.F. Agekyan, Spectroscopic properties of semiconductor crystals with direct forbidden energy gap, Phys. Stat. Solidi (a) 43, $11-42$ ( 1977).

60. S. Permogorov, Hot excitons in semiconductors, Phys. Stat. Solidi (b) 68, 9-42 (1975).

61. M. Cardona, Modulation Spectroscopy (New York - London, Academic Press, 1969).

62. S.I. Pekar, Crystal Optics and Additional Light Waves (Benjamin Cummings, Menlo Park, CA, 1983).

63. H. Haken, Quantum Field Theory of Solids (North-Holland, Amsterdam, 1976).

64. H. Haug and S.W. Koch, Quantum Theory of Optical and Electronic Properties of Semiconductors (World Scientific, London, 1993).

65. R.S. Knox, Theory of Excitons ( Academic Press, New York - London,1963).

66. V.G. Plekhanov, Application of Isotopic Effect in Solids (Springer, Heidelberg - Berlin, 2004).

67. G. Arfken, Mathematical Methods (Academic Press, New York - London, 1968).

68. A. Baldareshi, N.O. Lipari, Energy levels of direct excitons in semiconductors with degenerate bands, Phys.

Rev. B3, 439 - 451 (1971).

69. Excitons, E.I. Rashba and M.D. Sturge (eds.) (North - Holland, Amsterdam, 1982).

70. G.L.Bir and G.E. Picus, Symmetry and Deformation Effects in Semiconductors (Science, Moscow, 1972) (in Russian).

71. A.I.Ansel'm and Yu.A. Firsov, The pathway long of delocalized exciton in polar crystal, JETP (Moscow) 30, 719 - 722 (1956) (in Russian).

72. D.G. Thomas, ed. II -VI Semiconducting Compounds (Benjamin, New York, 1967).

73. Yu.A. Firsov, ed. Polarons (Moscow, Science, 1975) (in Russian).

74. Y. Toyozawa, Optical Processes in Solids, Cambridge (Cambridge University Press, 2003).

75. H. Frohlich, Electrons in lattice fields, Adv. Phys. 3, 325 - 361 (1954).

76. S.I. Pekar, Investigation of Crystal Electron Theory (Moscow, Science, 1951) (in Russian).

77. A.A. Klochikhin, Renormalization of Wannier - Mott exciton spectrum by Fröhlich interaction, Sov. Phys. Solid State 22, 986 - 992 (1980).

78. D.S. Bulyanitza, Exciton luminescence band shape from excited state of exciton, Phys. and Techn. Semiconductors 4, 1273 -1276 (1970) (in Russian).

79. V.G. Plekhanov, Isotopetronics and quantum information, Depon. in VINITI, N425 - B2011 (in Russian).

80. V.G. Plekhanov, Comparative study of isotope and chemical effects on the exciton states in LiH crystals, Prog. Sold State Chem. 29, 71 -177 (2001).

81. R. Zheng and M. Matsuura, Electron - phonon interaction in mixed crystals, Phys. Rev. B59, 15422 -15429 (1999).

82. M.J. Matsuura, Polaron in polyatomic crystals, J. Phys. C: Solid State Phys. 10, 3345 - 3350 (1977).

83. X. Wang and X.X. Liang, Electron-phonon interaction in ternary mixed crystals, Phys. Rev. B 42, 8915-8922 (1990).

84. R.J. Nicholas, R.A. Stradling, J.C. Ramage, Evidence for a reduction in the momentum matrix element $\mathrm{P}^{2}$ due to alloy disorder in $\operatorname{InAp}_{1-x} \mathrm{P}_{x}$, J. Phys. C: Solid State Phys.12, 1641- 1650 (1979).

85. S.J. Adachi, GaAs, AlAs, and $\mathrm{Al}_{x} \mathrm{Ga}_{1-x}$ As.Material parameters for use in research and device applicationsJ.J. Appl. Phys. 58, R1 - R30 (1985).

86. A.T. Collins, S.C. Lawson, G. Davies and H. Kanda, Indirect energy gap of ${ }^{13} \mathrm{C}$ diamond, Phys. Rev. Lett. 65, 891-894 (1990).

87. T.R. Anthony and W.F. Banholzer, Diamond properties as a function of isotopic composition, Thin Solid Films 212, 1 - 10 (1992).

88. N. Garro, A. Cantarrero, M. Cardons, A. Gobel, T Ruf and K. Eberl, Dependence of the lattice and the energy gap of zinc - blende - type semiconductors on isotopic masses, Phys. Rev. B54, 4732 - 4740 (1996); N. Garro, A. Cantarrero, M. Cardona, T. Ruf, A. Gobel, Ch. Lin, K. Reiman, M. Steube, Electron-phonon interaction at the direct gap of the copper halides, Solid State Commun. 98, 27- 30 (1996).

89. A. Gobel, T. Ruf, Ch. Lin, M. Cardona and J.C. Merle, Effect of isotopic composition on the lattice dynamics of CuCl, Phys. Rev. B56, 210 - 220 (1997).

90. V.F. Agekyan, V.M. Asnin, A.M. Kryukov, et al., Isotope effect in germanium, Fiz. Tverd. tela (St. Petersburg) 31, 101 - 104 (1989) (in Russian).

91. E.E. Haller, Isotope heterostructures selectively doped by neutron transmutation, Semicond. Sci. Technol. 5, 319 -321 (1990).

92. L.F. Lastras-Martinez, T. Ruf, M. Konuma and D.E. Aspnes, Isotopic effects on the dielectric response of Si around the $\mathrm{E}_{1}$ gap, Phys. Rev. B 61, 12946-12951 (2000).

93. J.M. Zhang, M. Giehler, A. Gobel, T. Ruf, M. Cardona, E.E. Haller and K. Itoh, Phys. Rev. B57, 1348 -1353 (1998); J.M. Zhang, T. Ruf and M. Cardona, Sulfur isotope effect on the excitonic spectra of CdS, Phys. Rev. B56, 9716 - 9720,1998.

$93^{a}$. T.A. Meyer, M.L.W. Thewalt, R. Lauck et al., Sulfur isotope effect on the excitonic spectra of CdS, Phys. Rev. B69, $115214-5(2004)$

94. F.I. Kreingol'd, K.F. Lider and L.E. Solov'ev, Isotope shift of exciton line in absorption spectrum $\mathrm{Cu}_{2} \mathrm{O}$, JETP Lett. : 23, 679 - 681 (1976) (in Russian). 
95. F.I. Kreingol'd, K.F. Lider and V.F. Sapega, Influence of isotope substitution on the forbidden gap of ZnO crystals, Fiz. Tverd. Tela 19, 3158 - 3160 (1977)(in Russian).

96. F.I. Kreingol'd, K.F. Lider and M.B. Shabaeva, Influence of isotope substitution sulfur on the exciton spectrum in CdS crystal, ibid 26, 3940 - 3941 (1984)(in Russian).

97. F.I. Kreingol'd, Dependence of band gap ZnO on zero - point energy, ibid 20, 3138 - 3839 (1978) (in Russian).

98. F.I. Kreingol'd and B.S. Kulinkin, Influence of isotope substitution on the forbidden gap of ZnO crystals, bid 28, 3164 - 3165 (1986) (in Russian).

99. A.I. Bobrysheva, I.I. Jeru and S.A. Moskalenko, The Isotopic shift of the excitonic absorption lines in $\mathrm{Cu}_{2} \mathrm{O}$ crystals, Phys. Stat. Solidi (b) 113, 439 - 445 (1982).

100. P. Etchegoin, J. Weber, M. Cardona, W.L. Hansen, K. Itoh and E.E. Haller, Isotope effect in Ge: a photoluminescence study, Solid State Commun. 83, 843 - 848 (1992).

101. G. Davies, E.C. Lihtowlerst, K. Itoh, W.. Hansen, E.E. Haller and V. Ozhogin, Isotope dependence of the indirect energy gap of germanium, Semicond. Sci. Technol. 7, 1271 - 1274 (1992).

102. V.G. Plekhanov, Isotope - induced energy - spectrum renormalization of the Wannier - Mott exciton in LiH crystals, Phys. Rev. B54, 3869 - 3877 (1996).

103. R.C. Buschert, A.E. A.E. Merlin, S. Pace, S. Rodriguez and M.H. Grimsditch, Effect of isotope concentration on the lattice parameter of germanium perfect crystals, Phys. Rev. B 38, 5219-5221 (1988).

104. U. Schmid and N.E. Christen, Calculated deformation potentials in Si, Ge, and GeSi, Solid State Commun. 75, 39 - 43 (1990).

105. S. Zollner, M. Cardona and S. Gopalan, Isotope and temperature shifts of direct and indirect band gaps in diamond-type semiconductors, Phys. Rev. B 45, 3376-3385 (1992).

106. G. Davies, E.C. Lightowlerst, T.S. Hui, V. Ozhogin, K. Itoh and E.E. Haller, Isotope dependence of the lowest direct energy gap in crystalline germanium, Semicond. Sci. Technol. 8, 2201 - 2204 (1993).

107. C. Parks, A.K. Ramdas, S. Rodriguez et al., Electronic band structure of isotopically pure germanium: Modulated transmission and reflectivity study, Phys. Rev. B 49, 14244-14250 (1994).

108. S. Tsoi, H. Alowadhi, X. Lu et al., Electron - phonon renormalization of electronic band gaps of semiconductors: Isotopically enriched silicon, Phys. Rev. B70, 193201 - 4 (2004).

109 A.K. Ramdas, S. Rodriguez, S. Tsoi et al., Electronic band gap of semiconductors as influenced by their isotopic composition, Solid State Commun. 133, 709 - 714 (2005).

110 S. Tsoi, S. Rodriguez, A.K. Ramdas et al., Isotopic dependence of the $\mathrm{E}_{0}$ and $\mathrm{E}_{1}$ direct gaps in the electronic band structure of Si, Phys. Rev. B72, 153203 - 4 (2005).

111 H. Kim, S. Rodriguez and T.R. Anthony, Electronic transitions of holes bound to boron acceptors in isotopically controlled diamond, Solid State Commun. 102, 861 - 865 (1997).

112. M. Cardona, Dependence of the excitation energies of boron in diamond on isotopic mass, Solid State Commun. 121, 7 - 8 (2002).

113. D. Karaskaja, M.L.W. Thewalt, T. Ruf et al., Photoluminescence studies of isotopically - enriched silicon: Isotopic effects on indirect electronic band gap and phonon energies, Solid State Commun. 123, 87 - 92 (2003).

114. A.A. Klochikhin and V.G. Plekhanov, Isotope effect on the Wannier - Mott exciton levels, Sov. Phys. Solid state 22, 1422- 1424 (1980) (in Russian).

115. V.G. Plekhanov, Direct observation of the effect of isotope - induced - disorder on exciton binding energy in $\mathrm{LiH}_{x} \mathrm{D}_{1-x}$ mixed crystals, J. Phys. Condens. Matter 19, 086221 - 9 (2007).

116. C.D. Clark, P.J. Dean and P.V. Harris, Intrinsic Edge Absorption in Diamond, Proc. R. Soc. (London) A277, 312 -319 (1964).

117. P.J. Dean, Inter-impurity recombinations in semiconductors, Progress in Solid State Chemistry 8, 1 - 126 (1973).

118. T. Ruf, M. Cardona P. Pavone et al., Cathodoluminescence investigation of isotope effects in diamond, Solid State Commun. 105, 311 - 316 (1991).

119. G. Herzberg, Molecular Spectra and Molecular Structure Spectra of Diatomic Molecules (New York, Van Nostrand, 1945).

120. A.F. Kapustinsky, L.M. Shamovsky, K.S. Bayushkina, Thermochemistry of isotopes, Acta Physicochim. (USSR) 7, $799-810$ (1937).

121. V.G. Plekhanov, Lattice dynamics of isotopically mixed crystals, Opt. spectrosc. (St. Petersburg) 82, 95 - 124 (1997).

122. A.A. Klochikhin and V.G. Plekhanov, Isotope effect on the Wannier - Mott exciton levels, Sov. Phys. Solid state 22, 342 - 344 (1980).

123. V.G. Plekhanov, Phonon renormalization of interband transition energy in the mixed crystals, Solid State Commun. 76, 51-53 (1990).

124. V.G. Plekhanov, Temperature and concentration dependence of exciton luminescence intensity, Opt. Spectr. 69, 1882 - 1889 (1990) (in Russian).

125. R.J. Nelson, 1982, Excitons in semiconductor alloys, in Excitons (Amsterdam, North-Holland, 1982) Chap. 8. 
126. R.J. Nelson, N. Holonjak and W. Groves, Free-exciton transitions in the optical absorption spectra of $\mathrm{GaAs}_{1-x} \mathrm{P}_{x}$, Phys. Rev. B 13, 5415-5419 (1976)

127. R. Monemar, K.K. Shih, G.D. Pettit, Some optical properties of the $\mathrm{Al}_{x} \mathrm{Ga}_{1-x}$ As alloys system, J. Appl. Phys. 47, $2604-2613$ (1976).

128. M.S. Brodin, N.I. Vitrikhovsky and A.A. Kipen, Exciton Reflection Spectra of Mixed Crystals $\mathrm{Zn}_{x} \mathrm{Cd}_{1-x} \mathrm{~S}$, (unpublished, 1982 , in Russian).

129. S.I. Radautsan, A.E. Tsurkan and A.E. Maksimova, Complex Semiconductors and Their Physical Properties (Kishinev, USSR, Stiinza, 1971) 12 (in Russian).

130. A.B. Kunz, D.J. Mikish, Electronic structure of LiH and NaH, Phys. Rev. B 11, 1700-1704 (1975).

131. G. Grosso, G. Pastori, SoliD State Physics (San Diego, Academic Press, 2000).

132. R.J. Elliott, J. Krumhansl and P. Leath, The theory and properties of randomly disordered crystals and physical systems, Rev. Mod. Phys. 46, 465543 (1974).

133. Y. Onodera, Y. Toyozawa, Persistence and amalgamation types in the electronic structure of mixed crystals, J. Phys. Soc. Japan 24, 341 - 355 (1968).

134. V.G. Plekhanov, A.V. Emelyanenko and A.U. Grinfelds, Excitonic structure of NaI and LiH crystals cleaved in liquid helium, Phys. Lett. A101, 291 - 293 (1984).

135. V.G. Plekhanov and V.I. Altukhov, Light scattering in LiH single crystals with LO phonons emission J. Raman Spectrosc. 16, 358 - 365 (1985).

136. H.Y. Fan, Temperature Dependence of the Energy Gap in Semiconductors, Phys. Rev. 82, 900-905 (1951); Photon-Electron Interaction: Crystal without Fields (Berlin, Springer-Verlag, 1967).

137. V.G. Plekhanov, Experimental manifestation of the effect of disorder on exciton binding energy in mixed crystals, Phys. Rev. B 53, 9558-9560 (1996).

138. V.G. Plekhanov, Fundamentals and applications of isotope effect in solids, Prog. Matter. Science 51, 287 - 426 (2006).

139. J. Sak, Effective Electron-Hole Interaction in Polar Semiconductors, Phys. Rev. B 6, 2226-2233 (1972).

140. S.D. Mahanti and C.M. Varma, Effective Electron-Hole Interactions in Polar Semiconductors, Phys. Rev.

B 6, 2209-2226 (1972).

141. A.B. Allen, Zero - point and isotope shift: relation to thermal shifts, Philos. Mag. B70, 527 - 534 (1995).

142. V.G. Plekhanov, Isotope effect on the lattice dynamics, Materials Sci. \& Eng. R35, 139 -237 (2001).

143. V.A. Kanehisa, R.J. Elliott, Effect of disorder on exciton binding in semiconductor alloys, Phys. Rev. B 35, 2228-2236 (1987).

144. N.F. Schwabe, R.J. Elliott, Approximation of excitonic absorption in disordered systems using a compositional - component weighted coherent - potential approximation, Phys. Rev. B54, 5318 - 5329 (1996).

145. D. Mahanti, Excitons in semiconducting alloys, Phys. Rev. B10, 1384 - 1390 (1974).

146. H.A. Bethe, E. Salpiter, Quantum Theory of One and Two Electron Automs (New York, Academic Press, 1957).

147. J. Hama, N. Kawakami, Pressure induced insulator - metal transiton in solid LiH, Phys. Rev. B39, 3351 - 3355(1988).

148. V.G. Plekhanov, Experimental manifestation of the effect of disorder on exciton binding energy in mixed crystals, Phys. Rev. B53, 9558 - 9560 (1996 - I)

149. V.G. Plekhanov, N.V. Plekhanov, Isotope dependence of band - gap energy, Phys. Lett. A313, 231 - 237 (2003).

150. V.G. Plekhanov, Comparative study of isotope and temperature effects involving excitons in $\mathrm{LiH}_{x} \mathrm{D}_{1-x}$ crystals, Phys. Solid State (St-Petersburg) 35, 1493 - 1499 (1993).

151 V.G. Plekhanov, Fundamentals and applications of isotope effect in modern technology, J. Nucl. Sci. Technol. (Japan) 43, 375 - 381 (2006).

152. L.D. Landau and E.M. Lifshitz, Quantum Mechanics (Nonrelativistic Theory) (New York, Pergamon Press, 1977).

153. A.R. Ubellohde, The Molten State of Substances, (Moscow, Metallurgy, 1982) (in Russian).

154. R. Loudon, The Raman effect in crystals, Adv. Phys. 13, 423 - 488 (1964).

155. R.A. Cowley, Anharmonicity, J. Phys. (Paris) 26, 659 - 664 (1965); Anharmonic crystals, Rep. Prog. Phys. 31, 123 - 166 (1968); in, Raman Effect, A. Anderson (ed.) (New York, Marcel Dekker, 1971).

156. M.A. Eliashevich, Mechanics of the vibrations of molecules, Uspekhi Fiz. Nauk (Moscow) 48, $482-544$ (1946) (in Russian).

157. D.A. Long, Raman Spectroscopy (UK, McGraw - Hill, Inc., 1977).

158. H. Hanzawa, N. Umemura, Y. Nisida and H. Kanda, Disorder effect of nitrogen impurities, irradiation induced defects and ${ }^{13} \mathrm{C}$ composition on the Raman spectrum in synthetic $\mathrm{I}^{b}$ diamond, Phys. Rev. B54, 3793 3799 (1996).

159. V.G. Plekhanov, Isotope Effects in Solid State Physics, in Semiconductors and Semimetals, Vol. 68, R.K. Willardson and E. Weber (eds.) (San Diego, Academic Press, 2001).

160. V.G. Plekhanov, Isotopical disorder in the Raman scattering spectra of crystals, Opt. Spectr. 78, 471 481 (1985).

161. R.M. Chrenko, ${ }^{13} \mathrm{C}$ - doped diamond: Raman spectra, Appl. Phys. 63, 5873 - 5875 (1988). 
162. K.C. Hass, M.A. Tamor, T.R. Anthony and W.F. Banholzer, Effect of isotopic disorder on the phonon spectrum of diamond, Phys. Rev. B44, 12046 - 12053 (1991).

163. S.H. Solin, A.K. Ramdas, Raman spectrum of diamond, Phys. Rev. B1, 1687 - 1699 (1970).

164. V.G. Plekhanov, Lattice - dynamics of isotope - mixed crystals, ArXiv, cond - mat/1007.5125 (2010).

165. R.J. Elliott, I.P. Ipatova (eds.) Optical Properties of Mixed Crystals (Amsterdam, North - Holland, 1988).

166. I.F. Chang, S.S. Mitra, Long - wavelength of optical phonons in mixed crystals, Adv. Phys. 20, 360 - 404 (1971).

167. V.G. Plekhanov, Lattice dynamics of isotopically mixed crystals, Opt. spectrosc. (St. Petersburg) 82, 95 - 124 (1997) (in Russian).

168. V.G. Plekhanov, Experimental evidence of strong phonon scattering in isotopical disordered systems: The case $\mathrm{LiH}_{x} \mathrm{D}_{1-x}$, Phys. Rev. B51, 8874 - 8878 (1995).

169. V.G. Plekhanov, Fundamentals and applications of isotope effect in modern technology, ArXiv: cond mat/0807.2521 (2008).

170. W. Cochran and R.A. Cowley, Phonons in perfect crystals, in, Encyclopedia of Physics, S. Flügge (ed), Vol 25/2 (Light and Matter) (Springer - Verlag, Berlin - Heidelberg - New York, 1967).

171. G.P. Srivastawa, The Physics of Phonons (Bristol, Hilger, 1990).

172. I.E. Tamm, Eine Bemerkung zur Diracschen Theorie der Lichtenstroung und Dispersion, Zs. Phys. 62, $705-708$ (1930).

173. M. Blackman, The specific heat of solids, in, Handbuch der Physik, S. Flüge (ed.) (Berlin, Springer Verlag, 1955) Vol 7, Pt. 1, p. $325-367$.

174. P. Klemens, Thermal Conductivity and Lattice Vibrational Modes, in, Solid State Physics, F. Seitz and

D. Turnbull (eds.) (New York, Academic Press, 1959) Vol. 7, p. 1 - 98.

175. P. Debye, The Debye theory of specific heat, Ann. Phys. (Leipzig) (4) 39, 789 - 803 (1912).

176. L.D. Landau and E.M. Lifshitz, Statistical Physics (New York, Pergamon Press, 1968).

177. G.L. Anderson, G. Nasise, K. Phillipson et al., Isotopic effects on the thermal expansion of lithium hydride, J. Phys. Chem. Solids 31, 613 - 618 (1970).

178. V.I. Ozhogin, A.V. Inyushkin, A.N. Taldenkov, et al., Isotope effect for thermal expansion coefficient of germanium, JETP (Moscow) 115, 243 - 248 (1999) (in Russian).

179. H. Jex, Thermal expansion and mode Gruneisen parameters of LiH and LiD, J. Phys. Chem. Solids 35, 1221 - 1223 (1974).

180. B.W. James, H. Kherandish, The low temperature variation of the elastic constants of LiH and LiD, J. Phys. C: Solid State Phys. 15, 6321 - 6339 (1982).

181. R.B. Von Dreele, J.G. Morgan, S.M. Stishov, Thermal expansion and equation of state of KCN of different isotopic composition, JETP (Moscow) 114, 2182 - 2186 (1998) (in Russian).

182. A.P. Zhernov, Thermal expansion of germanium crystal lattice with different isotope composition, Fiz. Tverd. Tela (St. Petersburg) 40, 1829 - 1831 (1998); Statistical displacement about isotope impurities and remain resistance, JETP (Moscow) 114, 2153 - 2165 (1998) (in Russian).

183. V.G. Plekhanov, Applications of isotope effects in solids, J. Materials Science 38, 3341 - 3429 (2003).

184. I.G. Kuleev, I.I. Kuleev, Influence normal processes of phonon - phonon scattering on maximum values of thermal conductivity isotope pure Si crystal, JETP (Moscow) 122, 558 - 569 (2002) (in Russian).

185. V.I. Tyutyunnik, Effect of isotope substitution on thermal expansion of LiH crystal, Phys. Status Solidi

(b) 172, 539 - $543(1992)$.

186. M. Planck, Zur Theorie der Wärmestrahlung, Ann Phys. 336, 758 - 768 (1910).

187. V.S. Kogan, Isotope effect in structuring properties, Sov. Phys. Uspekhi 5, 579 - 618 (1963).

188. P. Günter, Ann. Phys. 63, 476 (1920) cited in [94].

189. V.N. Kostryukov, The capacity of LiH between 3.7 and 295K, Zh. Fiz. Khim. (Moscow) 35, $1759-1762$ (1961) (in Russian).

190. G. Yates, G.H. Wostenholm, J.K. Bingham, The specific heat of ${ }^{7} \mathrm{LiH}$ and ${ }^{7} \mathrm{LiD}$ at low temperature, J. Phys. C: Solid State Phys. 7, 1769 - 1778 (1974).

191. M.W. Guinan, C.F. Cline, Room temperature elastic constants of ${ }^{7} \mathrm{LiH}$ and ${ }^{7} \mathrm{LiD}$, J. Nonmetals $\mathbf{1}, 11-15$ (1972).

192. D. Gerlich, C.S. Smith, The pressure and temperature derivatives of the elastic module of lithium hydride,

J. Phys. Chem Solids 35, 1587 - 1592 (1974).

193. T.H. Baron, J.G. Collins and G.K. White, Thermal expansion of solids at low temperatures, Adv. Phys.

29, 609 - 730 (1980).

194. V.G. Plekhanov, Lattice dynamics of isotope - mixed crystals, ArXiv:cond - mat/1007.5125.

195. Ch. Kittel, Thermal; Physics (Wiley, New York, 1969).

196. W. Schnelle and E. Gmelin, Heat capacity of germanium crystals with different isotopic composition, J.

Phys: Condens. Matter 13, 6087 - 6094 (2001).

197. M. Sanati and S.K. Estreicher, Specific heat and entropy of GaN, ibid 16, L327 - L331 (2004).

198. R. Peierls, Quantum Theory of Solids (Oxford, Clarendon Press, 1955).

199. J.M. Ziman, Models of Disorder (Cambridge, Cambridge University Press, 1979).

200. I.Ya. Pomeranchuk, About thermal conductivity of dielectrics, J. Phys. (USSR) 6, 237 - 246 (1942). 
201. T.H. Geballe, G.W. Hull, Isotopic and other types of thermal resistance in germanium, Phys. Rev. 110, $773-775$ (1958).

202. D.G. Onn, A. Witek, Y.Z. Qiu et al., Some aspect of the thermal conductivity of isotopically enriched diamond single crystals, Phys. Rev. Lett. 68, 2806 - 2809 (1992).

203. J.R. Olson, R.O. Pohl, J.W. Vandersande et al., Thermal conductivity of diamond between 170 and $1200 \mathrm{~K}$ and the isotopic effect, Phys. Rev. B47, 1485014856 (1993).

204. L. Wei, P.K. Kuo, R.L. Thomas, Thermal conductivity of isotopically modified single crystal diamond, Phys. Rev. Lett. 79, 3764 - 3767 (1993).

205. P. Debye, The Debye theory of specific heat, Ann. Phys. (Leipzig)4, 39, 789 - 803 (1912).

206. M. Cardona, R.K. Kremer, M. Sanati et al., Measurements of the heat capacity of diamond with different isotopic composition, Solid State Commun. 133, 465 - 468 (2005).

207. J. Callaway, Model for lattice thermal conductivity at low temperatures, Phys. Rev. 113, 1046 - 1051 (1959).

208. M. Asen - Palmer, K. Bartkowsky, E. Gmelin et al., Thermal conductivity of germanium crystals with different isotopic composition, Phys. Rev. B56, 9431 - 9447 (1997).

209. W.C. Capinski, H.J. Maris, S. Tamura, Analysis of the effect of isotope scattering on the thermal conductivity of crystalline silicon, Phys. Rev. B59, 10105 - 10110 (1999).

210. T. Ruf, R.W. Henn, M. Asen - Palmer et al., Thermal conductivity of isotopically enriched silicon, Solid State Commun. 115, 243 - 247 (2000); Erratum 127, 257 (2003).

211. A.P. Zhernov, A.V. Inyushkin, Kinetic coefficients in isotopically disordered crystals, Physics - Uspekhi (Moscow) 45, 573 - 599 (2002).

212. V.G. Plekhanov, Isotope engineering, Physics - Uspekhi (Moscow) 43, 1147 - 1154 (2000).

213. M. Omini, A. Sparavigna, Heat transport in dielectric solids with diamond structure, Nuovo Cimento D19, 1537 - 1563 (1997).

214. A. Sparavigna, Influence of isotope scattering on the thermal conductivity of diamond, Phys. Rev. B65, 064305 - 5 (2002), ibid, B67, 144305 - 4 (2003).

215. K.C. Hass, M.A. Tamor, T.R. Anthony and W.F. Banholzer, Lattice dynamics and Raman spectra of isotopically mixed diamond, Phys. Rev. B45, 7171 - 7182 (1992).

216. H.D. Fuchs, C.H. Grein, C. Thomsen et al., Comparison of the phonon spectra ${ }^{70}$ Ge and natural Ge crystals: Effect of isotopic disorder, ibid, B43, 4835 - 4841 (1991).

217. D.T. Wang, A. Gobel, J. Zegenhagen et al., Raman scattering on $\alpha$ - Sn: Dependence on isotopic composition, ibid, B56, 13167 - 13172 (1997).

218. S. Tamura, Isotope scattering of dispersive phonons in Ge, Phys. Rev. B27, 858 - 866 (1983).

219. V.G. Plekhanov, Applications of the Isotopic effect in Solids (Springer, Berlin - Heidelberg, 2004).

220. J.S. Blakemore, Semiconducting and other major properties of gallium arsenide, J. Appl. Phys. 53, R123 - R181 (1982).

221. J. Bylander, T. Duty and P. Delsing, Current measurement by real - time counting of single electrons, Nature 434, 361 - 364 (2005).

$221^{a}$. M.A. Kastner, The single electron transistor, Rev. Mod. Phys. 64, 849 - 858 (1992).

222. Y. Ono, A. Fujiwara, K. Nishiguch, et al., Manipulation and detection of single electrons for future information processing, J. Appl. Phys. 97, 031101 - 19 (2005)

223. H. Grabert and M.H. Devored (eds.), Single Charge Tunneling: Coulomb Blockade Phenomena in Nanostructures, NATO ASI Series B (New York, Plenum, 1992) Vol. 294; S. Washburn and R.A. Webb, Quantum transport in small disordered samples from the diffuse to the ballistic regime, Rep. Prog. Phys. 55, 1311 - 1383 (1992).

224. K. Barnham, D. Vvedensky, Low - Dimensional Semiconductor Structures (Cambridge, Cambridge University Press, 2009).

225. N. Gerasimenko, Ju. Parhomenko, Silicon - Material of Nanoelectronics (Technosphera, Moscow, 2007) (in Russian).

226. Special issue on Single Charge Tunneling, ed. by H. Grabert, Zs. Physik 85 (N3) (1991).

227. S. Washburn and R.A. Webb, Quantum transport in small disordered samples from the diffuse to the ballistic regime, Rep. Prog. Phys. 55, 1311 - 1383 (1992).

$227^{a}$. M.S. Brodin, V. Ya. Reznitchenko, Interactions of the laser intensity radiation with $\mathrm{A}_{2} \mathrm{~B}_{6}$ semiconductors, in Physics $\mathrm{A}_{2} \mathrm{~B}_{6}$ Compounds (A.N. Georgabiani and M.K. Sheinkman, eds, Science, Moscow, 1986) p. 184 - 225 (in Russian).

228. C.A. Klein, Further remarks on electron beam pumping lasers materials, Appl. Optics 5, 1922 - 1924

(1966); Power efficiency and quantum efficiencies of electron - beam pumped lasers, IEEE QE - 4, 186 - 194 (1968).

229. H. Haug, Theory of laser action involving free excitons and LO - phonon - assisted transitions, J. Appl. Phys. 39, 4687 - 4696 (1968); Nonlinear optical phenomena and bistability in semiconductors, Advances in Solid State Physics, Vol. 22, 149 - 171 (1982).

230. H. Haug and S. Koch,On the theory of laser action in dense exciton systems, Phys. Stat. Solidi (b) 82, $531-543(1977)$. 
231. D.V. Averin, A.A. Odintsov, S.A. Vyshenski, Ultimate accuracy of single - electron dc current standards, J. Appl. Phys. 73, 1297 - 1308 (1993).

232. O. Svelto, Principles of Lasers, $2^{\text {nd }}$ ed. ( New York, Plenum Press, 1982).

233. N.V. Karlov, Lectures on Quantum Electronics. (Science, Moscow, 1983) (in Russian).

234. W. Shockley, Electrons and Holes in Semiconductors ( Princeton, Van Nostrand Reinhold, 1950); J. Blakemore, Semiconductor statistics, $2^{\text {nd }}$ ed. (New York, Dover, 1985).

235. A.J. Taylor, D.J. Erskine and C.L. Tang, Ultrafast relaxation of photoexcited carriers in GaAs and related compounds, J. Opt. Soc. Am. (B) 2, 663 - 673 (1985).

236. J.A. Kash and J.C. Tsang, in Light Scattering in Solids, M. Cardona and G. Güntherodt (eds.) (Berlin, Springer-Verlag, 1991) Vol. 6, p. 423 - 467.

237. W.P. Dumke, Interband transitions and maser action, Phys. Rev. 127, 1559 -1563 (1962).

238. R.E. Nahory, K.L. Shakley, R.F. Leheny and R.A. Logan, Indirect - band - gap super - radiant laser in GaP containing isoelectronic traps, Phys. Rev. Lett. 27, 1647 - 1650 (1971).

239. H. Kressel, in Laser Handbook, F.T. Arechi and E.O. Schulz-Dubois (eds.) (Amsterdam, North-Holland, 1972) Chap. B5.

240. F. Stern, ibid, Chap. B4.

241. C. Klingshirn, Lasers processes in semiconductors, in Spectr. Solid - State Laser Type Matter (Proc. Course Enrico Fermi, Erice, NewYork - London, 1987) p 485 - 501.

242. K.C. Liu and R.L. Liboff, Criterion for exciton lasing in pure crystals, J. Appl. Phys. 54, 5633 -5637 (1983).

243. N.G. Basov, O.V. Bogdankevich, A.G. Devyatkov, Optical quantum generator on CdS crystals at the excitation high enrgy of electrons, JETP 20, 1588 - 11591 (1964) (in Russian).

244. N.G. Basov, O.V. Bogdankevich, A.G. Devyatkov, Some peculiarities of generation of the optical emission on CdS at electron excitation, Solid State Phys. (St. Petersburg) 8, 1536 - 1538 (1966) (in Russian).

245. J.R. Packard, D.A. Campbell, W.C. Tait, Evidence for indirect annihilation of free excitons in II-VI semiconductor lasers. J. Appl. Phys. 38, 5255-5258 (1967).

246. C. Benoit a la Guilaume, J.M. Debever, F. Salvan, in II-VI Semiconducting Compounds, ed. by D.G. Thomas (Benjamin, New York, 1967), p. 609 - 615.

247. C. Benoit a la Guilaume, J.M. Debever, F. Salvan, Radiative recmbination in highly excited CdS. Phys. Rev. 177, 567-580 (1969).

248. L.A. Kulevsky, A.M. Prokhorov, The nature of the laser transition in CdS crystal at 90K with two-photon excitaion. IEEE QE 2, 584-586 (1966).

249. M.S. Brodin, K.A. Dmitrenko, S.G. Shevel, L.V. Taranenko, The temperature dependence of laser threshold in CdS single crystals under one-photon excitation, in Proceedings of the

International Conference on Lasers'82 (STS Press, USA, 1983), pp. 287-291.

250. M.S. Brodin, S.V. Zakrevski, V.S. Mashkevich, V. Ya, Reznitchenko, On mechanism of generation of laser radiation in $\mathrm{CdS}_{x} \mathrm{CdSe}_{1-x}$ crystals in case of two-photon excitation. Sov. Phys. Semicond. 1, 595-597 (1967).

251. V.G. Plekhanov, Wannier-Mott excitons in isotope-disordered crystals. Rep. Prog. Phys. 61, 1045-1098 (1998).

252. K. Takiyama, M.I. Abd-Elrahman, T. Fujita and T. Okada, Photolumonescence and decay kinetics of indirect free excitons in diamonds under the near - resonant laser excitation, Solid State Commun. 99, 793 - 796 (1996).

253. V.G. Plekhanov and V.I. Altukhov, Free exciton luminescence and exciton - phonon interactions parameters of wide - gap insulators, in, Proc. Int. Conf. LASERS'82 (McClean, VA:STS, 1983) p. 292 - 299.

254. C. Klingshirn and H. Haug, Optical properties of highly excited direct gap semiconductors, Phys. Reports 70, $315-398$ (1981).

255. V.G. Plekhanov, Changes in spectra of luminescence and Raman scattering of lithium hydride under growth in the excitation intensity. Quantum Electron. (Moscow) 16, 2156-2159 (1989) (inRussian).

256. H.C. Casey, M.B. Panish, Heterostructure Lasers (Academic Press, New York, 1978).

257. P.S. Zoty, Quantum Well Lasers (Academic Press, Boston, 1993).

258. L.A. Colderen, S.W. Corzine, Diode Lasers and Photonic Integrated Cicuits (Wiley, New York, 1995).

259. P. Michler (ed.), Single Semiconductor Quantum Dots (Springer, Berlin, 2009)

$259^{a}$. N.N. Ledentsov, V.M. Ustinov, V.A. Shchukin et al., Quantum dot heterostructures: fabrication, properties, lasers. Fiz. Teh. Polup. (Phys. Tech. Semicond.) 32, 385-410 (1998) (in Russian).

260. L. Sirigu, D.Y. Oberli, L. Deriorgi et al., Excitonic lasing in semiconductor quantum wires. Phys. Rev. B61, R10575-4 (2000).

261. F. Rossi, E. Molinari, Linear and nonlinear optical properties of realistic quantum-wire structures: the dominant role of Coulomb correlation. Phys. Rev. B53, 16462-16473 (1996).

262. F. Rossi, G. Goldoni, E. Molinari, Theory of excitonic confinement in semiconductor quantum wires. J. Phys. Condens. Matter 11, 5969-5988 (1999). 